\title{
The macaque ventral intraparietal area has expanded into three homologue human parietal areas
}

\author{
Celia Foster ${ }^{1,2, *}$, Wei-An Sheng ${ }^{3 *}$, Tobias Heed ${ }^{1,2,4,5 \S}$, Suliann Ben Hamed $^{3,8}$ \\ ${ }^{1}$ Biopsychology \& Cognitive Neuroscience, Faculty of Psychology \& Sports Science, Bielefeld University, Bielefeld, Germany \\ ${ }^{2}$ Center of Cognitive Interaction Technology (CITEC), Bielefeld University, Bielefeld, Germany \\ ${ }^{3}$ Institut des Sciences Cognitives Marc Jeannerod, UMR5229, CNRS-University of Lyon 1 \\ ${ }^{4}$ Department of Psychology, University of Salzburg, Salzburg, Austria \\ ${ }^{5}$ Centre for Cognitive Neuroscience, University of Salzburg, Salzburg, Austria \\ "Celia Foster and Wei-An Sheng contributed equally. \\ ${ }^{\S}$ Tobias Heed and Suliann Ben Hamed contributed equally.
}

Contact: Tobias Heed tobias.heed@plus.ac.at, Suliann Ben Hamed, benhamed@isc.cnrs.fr

\begin{abstract}
Macaque ventral intraparietal area (VIP) in the fundus of the intraparietal sulcus has been implicated in a diverse range of sensorimotor and cognitive functions such as motion processing, multisensory integration, processing of head peripersonal space, defensive behavior, and numerosity coding. Here, we exhaustively review macaque VIP function, cytoarchitectonics, and anatomical connectivity and integrate it with human studies that have attempted to identify a potential human VIP homologue. We show that human VIP research has consistently identified three, rather than one, bilateral parietal areas that each appear to subsume some, but not all, of the macaque area's functionality. Available evidence suggests that this human "VIP complex" has evolved as an expansion of the macaque area, but that some precursory specialization within macaque VIP has been previously overlooked. The three human areas are dominated, roughly, by coding the head or self in the environment, visual heading direction, and the peripersonal environment around the head, respectively. A unifying functional principle may be best described as prediction in space and time, linking VIP to state estimation as a key parietal sensorimotor function. VIP's expansive differentiation of head and self-related processing may have been key in the emergence of human bodily self-consciousness.
\end{abstract}

Keywords: Posterior parietal cortex, human, macaque, functional homology, intraparietal area, VIP, cross-species, cortical expansion

\section{Introduction}

In both humans and non-human primates, the posterior parietal cortex (PPC) is divided into several distinct areas. In the macaque monkey, the most-studied non-human primate in neuroscience, these areas have been delineated based on cyto-, myelo-, and receptorarchitectonic criteria. These subdivisions coincide with specific structural connectivity patterns that have been characterized by anterograde and retrograde tracer injection as well as effective connectivity studies involving the combination of microstimulation and functional magnetic resonance imaging (fMRI). The different PPC areas are characterized by distinct functional properties as assessed with intra-cortical neuronal recordings and functional magnetic resonance imaging (fMRI). Together, the three criteria of i) cellular and receptor architecture; ii) connectivity, and iii) function result in a fine-grained parcellation of macaque PPC.

In macaques, PPC functional organization has been precisely mapped at multiple temporal and spatial scales using single cell recordings, fMRI, microstimulation, inactivation, and optogenetics. In addition, cytoarchitectonic studies have characterized the structural organization of macaque PPC. In contrast, the areal organization of PPC is presently less clear in humans. First, data about cellular and receptor architecture data is incomplete (Amunts et al., 2020; Niu et al., 2020). Second, human PPC connectivity is mostly assessed with functional connectivity methods, which do not allow us to distinguish between structural connectivity and connectivity that reflects complex functional interactions. Third, while human fMRI data is abundant, matching functional activation to anatomical regions is not straight-forward. These factors make a clear understanding of human PPC functional organization difficult. One strategy in attempting to delineate human PPC parcellation has been to assume functional homology between human and macaque PPC when interpreting human fMRI data. This strategy has been quite successful in describing how a human homologue of the macaque lateral intraparietal area (LIP) has diverged into multiple functional fields that share core properties with macaque LIP but are organized along a posterior-to-anterior axis of increasing functional complexity (Konen and Kastner, 2008a). Similarly, researchers have drawn parallels between many other human and macaque PPC areas, for instance the anterior intraparietal area (AIP), (Binkofski et al.,
1999; Cavina-Pratesi et al., 2007; Culham et al., 2003; Frey et al., 2005) and area V6A (Pitzalis et al., 2013b, 2015). Yet, homology is less evident for other regions of PPC. The ventral intraparietal area (VIP) lies centrally in PPC, at the fundus of the macaque intraparietal sulcus. Whereas macaque VIP is clearly defined by the convergence of specified anatomical and functional properties, the definition of a human VIP homologue has varied across studies. This has led to a lack of consensus as to where VIP may be located in the human brain. In this review, we will lay out the definition and functional characteristics of VIP in the macaque and the different approaches that have attempted to define its human homologue. We will present an exhaustive overview of the research into macaque and human VIP, organized along the different functional processes with which the area has been associated. We include a brief overview of the anatomical networks which are thought to mediate each respective function together with VIP. Our key finding is that not one, but three human PPC regions likely process the functions that VIP mediates in the macaque. Based on the integration of anatomical, functional and connectivity considerations, we will propose a precise definition of a human putative VIP complex with three individual areas which we term pVIP\#1-\#3 and discuss the possible overarching role of this region in the larger context of PPC.

\subsection{Definition of macaque area VIP}

The name ventral intraparietal area (VIP) was coined by Maunsell \& van Essen (1983) who reported a projection from the macaque middle temporal area (MT) that terminated in the fundus of the intraparietal sulcus (IPS). A projection from the medial superior temporal area (MST) was also later described (Boussaoud et al., 1990). The region's boundaries and its neighbors were subsequently defined via cyto-architectonic mapping (Lewis and Van Essen, 2000a; see Fig. 1). Accordingly, the definition of macaque VIP relies on a specific layer and cellular organization and a specific connectivity pattern with dorsal stream extrastriate visual areas. This definition coincides with distinct functional properties relative to neighboring parietal regions: VIP neurons exhibit direction-selective responses to large field visual motion stimuli (Colby et al., 1993), somatosensory responses to head and shoulder stimulation, and visual responses to stimuli close to these body parts (Duhamel et al., 1997). VIP was further divided into lateral (VIP1) and medial (VIPm) 


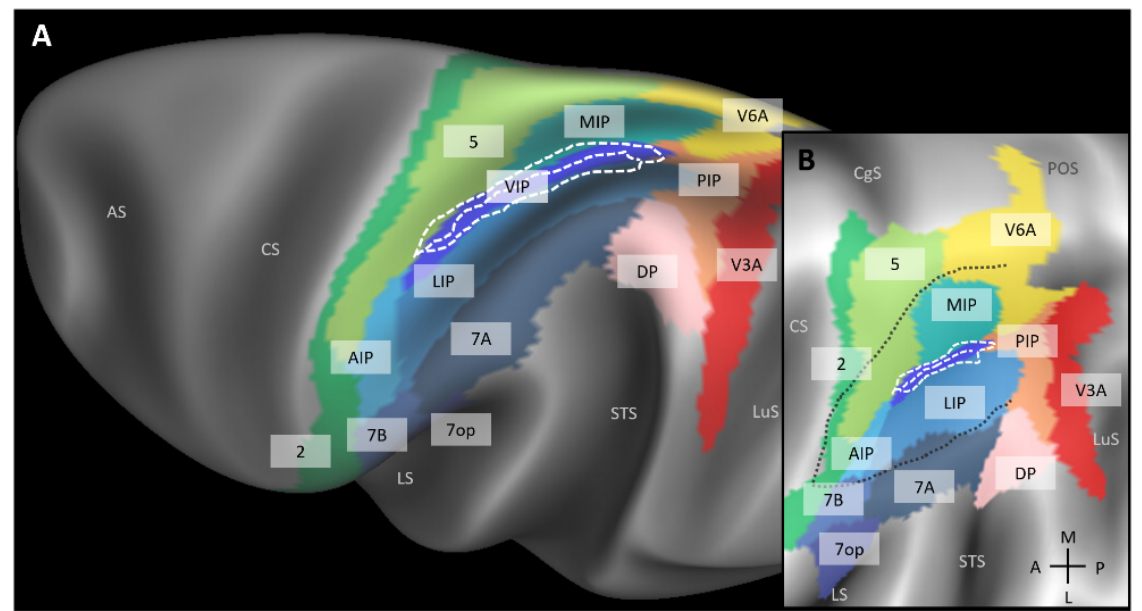

Figure 1. Location of macaque VIP and surrounding cortical areas. (A) Location of VIP (dark-blue color) and surrounding areas as defined by Markov et al. (2014). The white dashed contour reflects the cyto-architecturally defined VIP (Lewis and Van Essen, 2000a) and its subdivsions VIPm (bordering MIP and area 5) and VIPl (bordering LIP). All regions were co-registered on an inflated monkey brain using Connectome Workbench version 1.4.2. (B) Flat map of the intraparietal sulcus (black dashed contour) with the same cortical areas as in A. AS: arcuate sulcus, CS: central sulcus, LS: lateral sulcus, STS: superior temporal sulcus, LuS: lunate sulcus, CgS: cingulate sulcus, POS: parieto-occipital sulcus. 2: area 2, 5: area 5, 7a: area 7a, 7b: area 7b, 7op: opercular area 7, AIP: anterior parietal area, DP: dorsal prelunate area, LIP: lateral intraparietal area, MIP: medial intraparietal area, PIP: posterior parietal area, VIP: ventral intraparietal area, V6A: extrastriate visual area V6A, V4A: extrastriate visual area V3A.

parts based on differences regarding myelination (Lewis and Van Essen, 2000a) and receptor profiles (Niu et al., 2020). However, little if anything is known about the functional differences of these two subdivisions.

\subsection{An (attempted) definition of the ventral intraparietal area (VIP) in humans}

Efforts to define the human homologue of macaque VIP have focused on circumscribed functional and cytoarchitectonic aspects acquired in human studies to probe interspecies functional homology. Table 1 lists the Montreal Neurological Institute (MNI) coordinates reported by studies that have claimed to have identified a putative human VIP in human fMRI studies. Three studies have sought a strict functional homology between monkey and human VIP. Each of them capitalized on a unique subset of macaque VIP functional responses, and later human studies have often used one of the three approaches to localize human VIP for further characterization (see Fig. 2A). The first functional approach (\#1) identifies PPC activations to egomotion-consistent visual motion as opposed to scrambled and local motion (Fig. 2A, orange, Wall \& Smith, 2008). The second approach (\#2) relies on the conjunction of brain responses to visual, tactile, and auditory motion stimuli compared to static control stimuli (Fig. 2A, red, Bremmer et al., 2001). The third approach (\#3) is based on the topographic mapping of tactile stimuli on, and visual stimuli close to, the face (Fig. 2A, purple, Sereno \& Huang, 2006). Approaches \#1 and \#2 rely on large field moving stimuli, whereas approach \#3 topographically maps out tactile stimuli across the skin using airpuffs with controlled pressure, and visual stimuli close to the face using phase-encoded retinotopic mapping procedures that employ natural movie stimuli. Moreover, approach \#1 regards visual information as central for VIP, whereas approaches \#2 and \#3 view VIP as a multisensory area. Critically, the parietal activations of the three localizer methods are distributed over a large extent of the human PPC. Moreover, we will show that many putative VIP locations suggested by other human fMRI studies cluster around the three locations defined by experimental approaches \#1-\#3. Therefore, we will refer to these larger clusters as putative VIP (pVIP) \#1-\#3 from hereon. Fig. 2A presents tentative outlines for these three clusters; we emphasize that these outlines simply represent an illustration drawn around the multiple previously reported average locations and do not reflect anatomically or functionally well-defined areas.

Cytoarchitectonic mapping of the human IPS and superior parietal lobule has identified a large number of distinct regions (Richter et al., 2019; Scheperjans et al., 2008). However, there is no consensus about the functional correspondence of any of these regions to macaque VIP. One study found that area 7PC, a cytoarchitectonic region in the human superior parietal lobule (Scheperjans et al., 2008), showed resting state connectivity with area V5/MT, dorsal and ventral premotor cortex (PMd, PMv) and the frontal eye fields (FEF), regions known to be connected with macaque VIP (Mars et al., 2011). This suggested that human VIP relocated from the IPS to the superior parietal lobule, consistent with other findings that VIP's neighboring regions, while mostly confined to the IPS in the macaque, are found outside the IPS in the human brain (Kastner et al., 2017), possibly due to the expansion of the parietal cortex in the human brain (Van Essen et al., 2012a, 2012b). However, another human cytoarchitectonic mapping study identified a comparable number and topographical arrangement of human IPS regions as in macaques. In their scheme, human VIP corresponds to human intraparietal area 1 (hIP1), located in the anterior IPS at a location strictly homologous to that of macaque VIP (Richter et al., 2019).

Fig. 2B illustrates the overlap of the three pVIP clusters with human cytoarchitectonic regions. Activations identified with approach \#1 overlapped with areas 7A, hIP3 and hIP1 in both hemispheres, with area 7P in the right hemisphere and hIP6 in the left hemisphere. Activations evoked by approach \#2 overlapped with area 7PC bilaterally, with hIP2 and area 2 in the left hemisphere and 7A and hIP3 in the right hemisphere. Activations identified with approach \#3 overlapped with area 7A bilaterally, with area 2 in the left hemisphere and area 7PC in the right hemisphere. Notably, pVIP\#2 and \#3 showed overlap with area 7PC, the cytoarchitectonically defined VIP in the superior parietal sulcus (Mars et al., 2011; Scheperjans et al., 2008) and only pVIP\#1 showed a small overlap with hIP1, the proposed cytoarchitectonically defined VIP in the IPS (Richter et al., 2019). For all three approaches, the cytoarchitectonicfunctional match was inconsistent across hemispheres. These differences may be primarily driven by the known differences in the cytoarchitectonic maps of PPC across hemispheres (Scheperjans et al., 2008). The functional relevance of these hemispheric differences is not currently known.

The picture becomes even more complex when we consider proposed human VIP locations defined by less stringent functional criteria or by higher order cognitive tasks that have not been investigated in monkeys (see list of studies in Table 1; Fig. 3). Pooling these reported locations does not identify a human putative VIP hotspot; rather, proposed VIP loci spread all over human anterior IPS. Some of them lie close to the cytoarchitectonically defined VIP in the superior parietal sulcus (Mars et al., 2011; Scheperjans et al., 2008; see Fig. 3 , yellow star symbol). Others lie close to the cytoarchitectonically defined VIP in the anterior fundus of the IPS (Richter et al., 2019; see Figure 3, yellow pentagon symbol) and areas hIP3 (Fig. 3, yellow diamond) and 7A (Fig. 3, yellow triangle). Yet again others are near neither location.

In sum, there is no firm knowledge about the potential homology of monkey VIP with a human PPC region. In an attempt to synthetize and clarify our current view of human VIP, the next section will provide a systematic functional comparison between monkey and human VIP studies. 


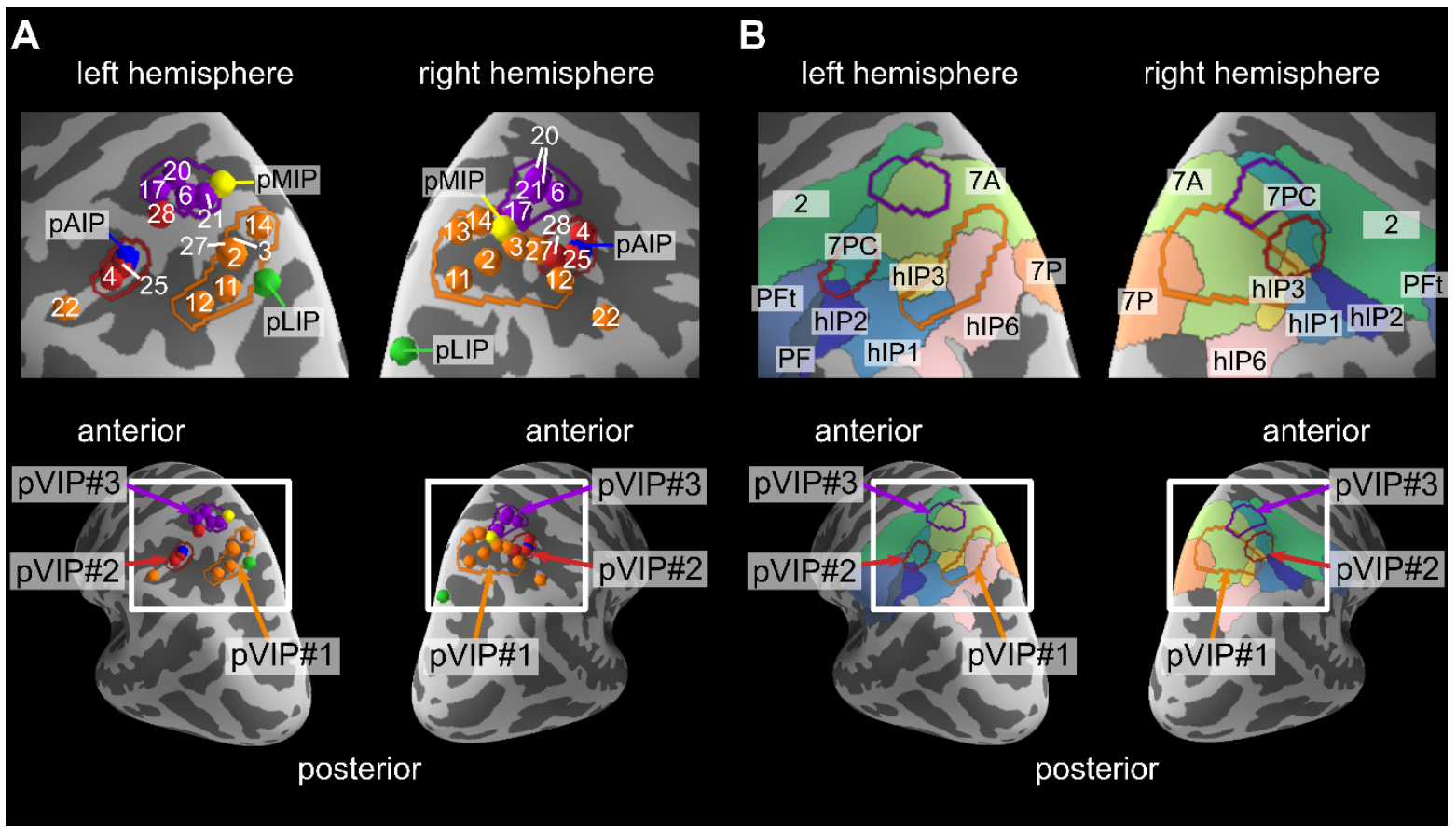

Figure 2. Proposed locations of putative human VIP (pVIP) as defined using the three most common methods; (pVIP\#1 - orange) higher activation by egomotion-consistent visual motion compared to scrambled motion (Wall and Smith, 2008), (pVIP\#2 - red) the conjunction of responses to visual motion, tactile motion and auditory motion stimuli compared to static control stimuli (Bremmer et al. MNI space) that are defined topographic mapping of tactile stimuli on the face and visual stimuli close to the face (Sereno and Huang, 2006). (A) Each sphere shows the locations of pVIP coordinates (in arbitrarily drawn to surround the locations reported in this figure, and we have excluded study 22 in both hemispheres and study 28 on the left hemisphere as these coordinates appear to be outliers. As arbitrarily drawn to surround the locations reported in this figure, and we have excluded study 22 in both hemispheres and study 28 on the left hemisphere as these coordinates appear to be outliers. As
comparison points, we show mean locations of putative human LIP (Konen and Kastner, 2008; Medendorp et al., 2003; Sereno et al., 2001), AIP (Frey et al., 2005) and MIP (Grefkes et al., 2004; Prado comparison points, we show mean locations of putative human LIP (Konen and Kastner, 2008; Medendorp et al., 2003; Sereno et al., 2001), AIP (Frey et al., 2005) and MIP (Grefkes et al., 2004; Prado
et al., 2005), with each mean coordinate generated by averaging over the reported locations in these studies. (B) Locations of the three pVIP clusters in comparison to cytoarchitectonic regions in the intraparietal and superior parietal cortex (Amunts et al., 2020; Richter et al., 2019; Scheperjans et al., 2008). Code and data used to generate the human figures is available at: https://osf.io/nce7x/.

\section{Functional comparison between macaque} monkey and putative human VIP

This section provides a systematic comparison of all monkey and human VIP studies we identified via keyword searches in PubMed and Google Scholar. Table 2 summarizes the plethora of functions that have been associated with VIP in macaques and humans. In Section 2.1, we cover the processing of multiple sensory modalities - vision, vestibular sensation, proprioception, touch, and audition in VIP. Already in these passages, it will become evident that VIP performs complex integrative functions. For clarity, we separately address multisensory functionality in Section 2.2. Section 2.3 addresses VIP's potential (sensori)motor functions, and we address the reference frames used by VIP in Section 2.4. Finally, the functions provided by VIP are likely also exploited for "higher" cognition, and we turn to these properties in Section 2.5 .

Not all VIP functions have been probed in both species due to methodological limitations. For example, single neuron responses have been exclusively explored in macaques, but embodiment and body ownership has been almost exclusively investigated in humans. Unsurprisingly, macaque studies have mostly focused on low-level functional properties, whereas human studies have more often tried to associate VIP with higher order functions. With the current lack of consensus about the existence and/or location of human VIP, we use the term "putative VIP" (pVIP) to denote the presently preliminary name assignment. Moreover, we will relate previous work to pVIP\#1, pVIP\#2, and pVIP\#3 (see Fig. 2) whenever possible. When we refer to all three regions together, we will use the name "pVIP complex".

\section{$2.1 \quad$ Unimodal sensory modalities}

\subsubsection{Vision}

Over-representation of central near visual space. Once identified through connectivity, VIP was physiologically characterized as exhibiting strong selectivity for both the direction and speed of moving visual stimuli in single neuron responses (Colby et al., 1993). In addition, some VIP neurons are selective for the distance at which a stimulus is presented (Cléry et al., 2018; Colby et al., 1993), and some neurons respond best to movement toward the animal regardless of absolute direction (Bremmer et al., 2013; Cléry et al., 2017; Colby et al., 1993). Therefore, the receptive fields (RFs) of VIP neurons should be viewed as a 3D, rather than a 2D, property. Overall, VIP RFs over-represent central visual space, although they also cover the periphery of the visual field (Viswanathan and Nieder, 2017a), including the far periphery (Bremmer et al., 2002a; Duhamel et al., 1997). Specifically, RFs extend up to $54^{\circ}$ laterally, with a peak in eccentricity at $20^{\circ}$, however their average center of mass is $\sim 10^{\circ}$ (Ben Hamed, 1999), as they globally over-represent near space, despite responding to both far and near stimuli (Bremmer et al., 2013; Cléry et al., 2018; Colby et al., 1993).

Regarding macroscopic organization, VIP visual RFs are not organized along a clear topographic map, contrary to striate and extrastriate visual areas. This is consistent with the proposition that local retinotopic topography vanishes with hierarchical distance from visual cortical input (Viswanathan and Nieder, 2017b). In addition, the lack of any obvious visual-topographical organization fits the visuo-spatial tuning of VIP neurons, which ranges from retinotopic to craniotopic (Duhamel et al., 1997).

In humans, visual stimulation close to the face identifies a cortical region that shares some of the core sensory properties of macaque VIP (Huang et al., 2017; Sereno and Huang, 2006). This finding has led to the proposal that the corresponding region, $\mathrm{pVIP} \# 3$ (see Fig. 2 ), is the human VIP homologue (Sereno and Huang, 2014). We will discuss this work further in the section on peripersonal space. Here, we note that this research has uncovered orderly, tactile and visual maps in the putative human VIP - a finding that implies that neurons are grossly arranged according to their (tactile or visual) spatial characteristics. Analogous fMRI mapping experiments have not yet been undertaken in macaques.

Sensitivity to optic flow stimuli. Despite the relative dominance of central space in VIP, the area responds to large field optic flow stimuli. Optic flow motion patterns on the retina often result from self-motion, so VIP has been associated with the detection of egomotion cued by optic flow. In line with this idea, VIP receives dense inputs from the dorsal part of the medial superior temporal area (MSTd), an area sensitive to first-order translation, divergence, convergence and rotation optic flow components (Duffy and Wurtz, 


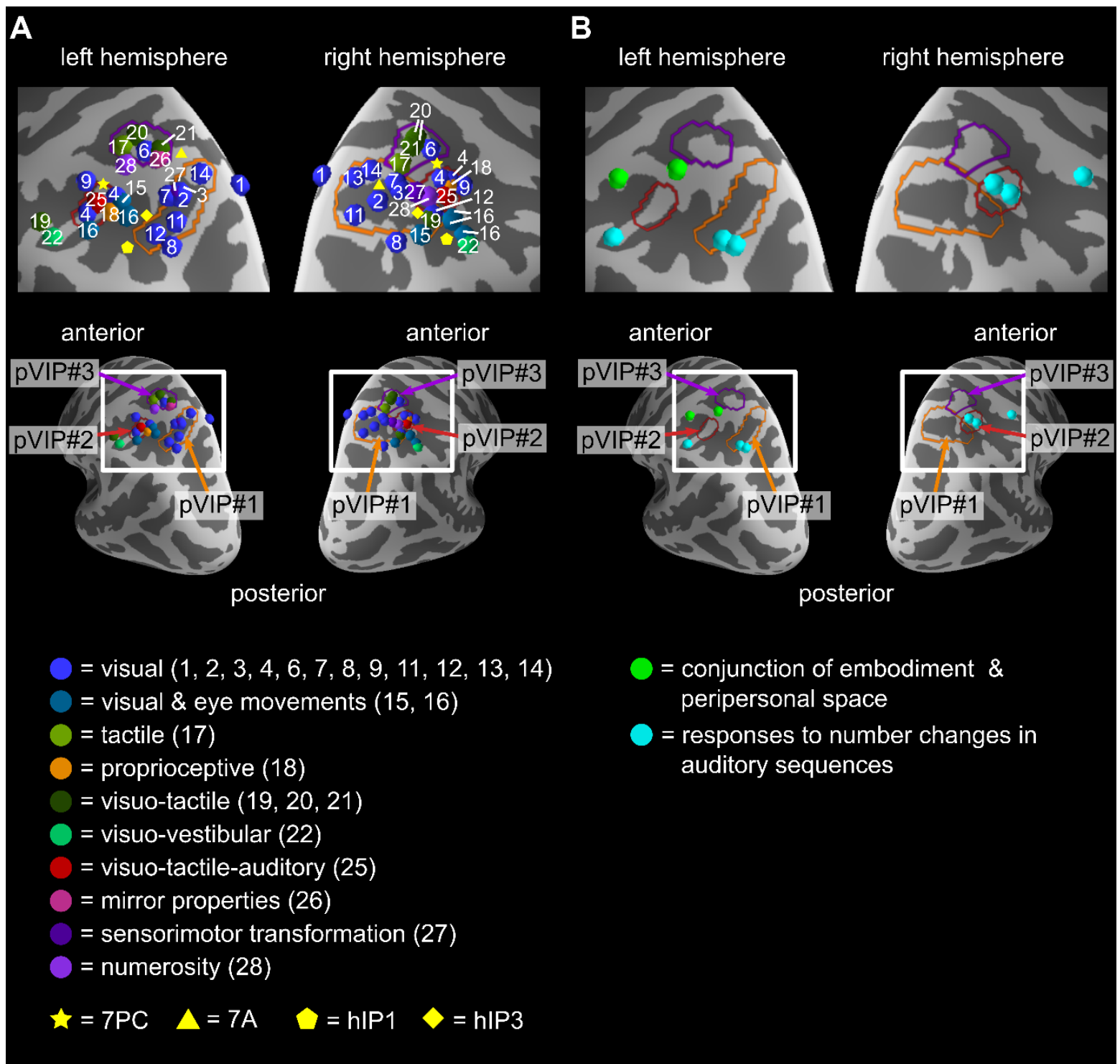

Figure 3. Locations of putative human VIP (pVIP) coordinates and other activations that may correspond to pVIP. (A) Proposed locations of pVIP coordinates (in MNI space) given in all studies we identified that explicitly proposed a human homologue VIP region (see Table 1; number labels refer to the study number in left-most column). The color of each sphere refers to the sensory modality/modalities or functional process that was investigated in the respective study. For comparison, we include the three $p$ VIP cluster outlines from Fig. 2, yellow stars to show the position of area
7PC, yellow triangles to show the position of 7A, yellow pentagons to show the position of hIP1 and yellow diamonds to show the position of hIP3. (B) Locations of coordinates (in MNI space) that may correspond to pVIP. Green spheres show the conjunction of responses to peripersonal space and body ownership from a meta-analysis (Grivaz et al., 2017) and cyan spheres show responses to number changes in auditory sequences (Wang et al., 2015).

1991; Lagae et al., 1994). VIP neurons are particularly sensitive to expansion, the dominant optic flow component that arises from active whole body displacement (Schaafsma and Duysens, 1996). During natural motion, expansion cues depend on body motion direction as well as on head position relative to the body. Indeed, VIP neurons are strongly modulated by the experimental manipulation of cues that reflect head-on-body posture, for instance by shifting the center of the optic flow (Bremmer et al., 2002a; Zhang and Britten, 2010, 2011). In addition, VIP neural responses differ depending on whether motion patterns can or cannot be produced by egomotion (Cottereau et al., 2017; Pitzalis et al., 2021). From a computational point of view, VIP neurons are sufficiently sensitive to support heading judgement (Zhang and Britten, 2010), as is the case for area MSTd (Ben Hamed et al., 2003). All these findings support a putative involvement of VIP in egomotion perception.

However, local reversible inactivation of area VIP did not result in observable deficits in perceptual heading thresholds (Chen et al., 2016), whereas MSTd inactivation did (Gu et al., 2012). This result may suggest that VIP receives copies of egomotion signals for some yet undefined purpose, but is not itself involved in their computation - reminiscent of a long-standing debate about the neighboring area LIP, which shows strong evidence of accumulation signals during perceptual decisions, but whose inactivation leaves decision behavior largely unaffected (Katz et al., 2016). It is important to note that neural responses to optic flow in area VIP differ during active and passive contexts. The preferred directions of neurons differ during active eye movements, such as smooth pursuit, and static fixation (Gabel et al., 2002). More so, VIP microstimulation causally changes heading perception, and this effect is stronger during active eye smooth pursuit than fixation (Zhang and Britten, 2011). Surprisingly, however, the amplitude of neuronal activity during externally induced motion stimulation was larger than when selfinduced (Churan et al., 2021). The above-mentioned inactivation study tested perceptual heading thresholds under fixation, and different results may be obtained under other experimental protocols (Chen et al., 2016). 
Table 1. List of fMRI studies that have proposed average brain coordinates for putative human VIP (pVIP). Coordinates are given in MNI space and are illustrated in Fig. 3a. * indicates that Talairach coordinates were given in the study, which we transformed to MNI space using the BioImage Suite converter http://sprout022.sprout.yale.edu/mni2tal/mni2tal.html (Lacadie et al., 2008). Note that we include studies that have named pVIP as the Intra-Parietal Sulcus motion region (IPSmot) as this nomenclature was used due to the authors noting that a variety of locations have been defined as pVIP, and that there is an uncertainty in defining a human VIP without directly comparing homology between macaque and human fMRI responses (Pitzalis et al., 2013a).

\begin{tabular}{|c|c|c|c|c|c|}
\hline & No & Study & pVIP MNI coordinates & Definition of pVIP & Study approach and VIP-related findings \\
\hline \multirow{14}{*}{ 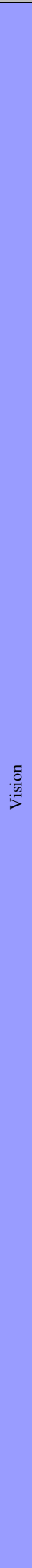 } & 1 & Bartels et al., (2008) & $\begin{array}{l}\text { Left: }-4,-64,60 \\
\text { Right: } 6,-66,58\end{array}$ & $\begin{array}{l}\text { A region in the medial posterior } \\
\text { parietal cortex that responded to global } \\
\text { flow motion in a natural movie } \\
\text { stimulus. }\end{array}$ & $\begin{array}{l}\text { Study investigating the responses to visual motion during } \\
\text { natural movie viewing. pVIP responded to global flow } \\
\text { motion and showed invariance to visual contrast and } \\
\text { connectivity with anterior V5/MT+. }\end{array}$ \\
\hline & 2 & $\begin{array}{l}\text { Cardin and Smith, } \\
(2010)\end{array}$ & $\begin{array}{l}* \text { Left: }-25,-59,55 \\
\text { *Right: } 25,-60,53\end{array}$ & $\begin{array}{l}\text { Higher responses to egomotion- } \\
\text { consistent visual motion than } \\
\text { egomotion- inconsistent, but coherent, } \\
\text { visual motion. }\end{array}$ & $\begin{array}{l}\text { Study investigating the responses in visual and vestibular } \\
\text { regions to egomotion-consistent visual motion compared } \\
\text { to egomotion-inconsistent, but coherent, visual motion. }\end{array}$ \\
\hline & 3 & $\begin{array}{l}\text { Di Marco et al., } \\
(2021)\end{array}$ & $\begin{array}{l}\text { Left: }-26,-59,56 \\
\text { Right: } 27,-55,56\end{array}$ & $\begin{array}{l}\mathrm{pVIP} \text { was termed IPSmot. Contrast of } \\
\text { coherent optic flow motion with } \\
\text { random visual motion. }\end{array}$ & $\begin{array}{l}\text { Study investigating the responses to visual optic flow } \\
\text { motion that simulated forward or backward motion on a } \\
\text { curved or linear path. pVIP/IPSmot showed stronger } \\
\text { responses to curved path visual motion as compared to } \\
\text { linear motion. }\end{array}$ \\
\hline & 4 & Field et al., (2020) & $\begin{array}{l}\text { pVIP defined by } \\
\text { functional localizer: } \\
\text { Left: }-34,-44,42 \\
\text { Right: } 38,-40,52 \\
\text { pVIP defined by a } \\
\text { whole-brain group } \\
\text { contrast of object-motion } \\
\text { > self-motion: } \\
\text { Left: }-34,-48,50 \\
\text { Right: } 36,-46,56\end{array}$ & $\begin{array}{l}\text { Functional localizer based on the } \\
\text { method of Bremmer et al., (2001), the } \\
\text { conjunction of higher responses to } \\
\text { visual, tactile and auditory motion as } \\
\text { compared to static control stimuli } \\
\text { within each sensory modality. }\end{array}$ & $\begin{array}{l}\text { Study investigating the neural responses to simulated } \\
\text { object-motion and self-motion visual stimuli. pVIP } \\
\text { showed stronger responses to object-motion stimuli } \\
\text { compared to self-motion stimuli. }\end{array}$ \\
\hline & 5 & Furlan et al., (2014) & Coordinates not reported. & $\begin{array}{l}\text { Higher responses to egomotion- } \\
\text { consistent visual motion than } \\
\text { egomotion- inconsistent, but coherent, } \\
\text { visual motion as in Cardin \& Smith, } \\
\text { (2010); Wall \& Smith, (2008). }\end{array}$ & $\begin{array}{l}\text { Study investigating the neural responses to changes in } \\
\text { heading direction in visual egomotion stimuli. pVIP } \\
\text { showed stronger responses to stimuli that implied changes } \\
\text { in heading direction compared to control stimuli with no } \\
\text { heading direction change. Furthermore, the direction of } \\
\text { heading direction change could be decoded from pVIP } \\
\text { activity. }\end{array}$ \\
\hline & 6 & Huang et al., (2015) & $\begin{array}{l}\text { *Left: }-30,-46,65 \\
\text { *Right: } 30,-44,60\end{array}$ & $\begin{array}{l}\text { Functional wide-field motion localizer, } \\
\text { contrasting structured motion with } \\
\text { scrambled motion (Sereno and Huang, } \\
\text { 2006). }\end{array}$ & $\begin{array}{l}\text { Study investigating neural responses to passive and active } \\
\text { avoidance of obstacles. Subjects viewed a hallway virtual } \\
\text { reality environment and either actively dodged obstacles } \\
\text { using a button press or passively observed obstacles being } \\
\text { dodged. pVIP showed no difference in activation during } \\
\text { passive vs active dodges. }\end{array}$ \\
\hline & 7 & $\begin{array}{l}\text { Quinlan and } \\
\text { Culham, (2007) }\end{array}$ & $\begin{array}{l}\text { *Left: }-27,-56,55 \\
\text { *Right: } 25,-54,58\end{array}$ & $\begin{array}{l}\text { Functional localizer, higher responses } \\
\text { to looming and receding visual motion } \\
\text { of a ball towards the face, as compared } \\
\text { to a stationary presented ball. }\end{array}$ & $\begin{array}{l}\text { Study investigating the neural responses to near-, mid- and } \\
\text { far-distance visual stimuli. pVIP did not show differences } \\
\text { in activation to near vs far stimuli. }\end{array}$ \\
\hline & 8 & $\begin{array}{l}\text { Pitzalis et al., } \\
(2013 a)\end{array}$, & $\begin{array}{l}\text { Left: }-30,-60,45 \\
\text { Right: } 30,-60,45\end{array}$ & $\begin{array}{l}\text { pVIP was termed IPSmot. Higher } \\
\text { responses to visual motion compared to } \\
\text { a static visual condition. }\end{array}$ & $\begin{array}{l}\text { Study investigating the responses to different kinds of } \\
\text { visual optic flow motion in motion-responsive brain } \\
\text { regions. pVIP/IPSmot showed strongest responses to } \\
\text { translation motion, as well as stronger responses to spiral } \\
\text { motion than to random motion. }\end{array}$ \\
\hline & 9 & Pitzalis et al., (2020) & $\begin{array}{l}\text { Left: }-30,-40,49 \\
\text { Right: } 33,-40,49\end{array}$ & $\begin{array}{l}\text { pVIP was termed IPSmot. Higher } \\
\text { responses to visual motion compared to } \\
\text { a static visual condition. }\end{array}$ & $\begin{array}{l}\text { Study investigating the responses to pure and combined } \\
\text { self- and object-motion conditions where subjects viewed } \\
\text { natural movies of a moving train. pVIP/IPSmot showed } \\
\text { stronger responses to combined self- and object-motion } \\
\text { conditions as compared to pure self- or object-motion only } \\
\text { conditions. }\end{array}$ \\
\hline & 10 & $\begin{array}{l}\begin{array}{l}\text { Sulpizio } \\
(2020)\end{array} \\
\text { et }\end{array}$ & Coordinates not reported & $\begin{array}{l}\text { pVIP was termed IPSmot. Contrast of } \\
\text { coherent visual motion with random } \\
\text { visual motion. }\end{array}$ & $\begin{array}{l}\text { Study investigating the responses to optic flow vs. random } \\
\text { visual motion in scene-responsive regions and scene vs. } \\
\text { face responses in visual motion-responsive brain regions. } \\
\text { pVIP/IPSmot showed no difference in its responses to } \\
\text { scene and face images. }\end{array}$ \\
\hline & 11 & $\begin{array}{l}\text { Uesaki and Ashida, } \\
\text { (2015) }\end{array}$ & $\begin{array}{l}\text { *Left: }-23,-59,48 \\
\text { *Right: } 19,-62,50\end{array}$ & $\begin{array}{l}\text { Functional localizer, higher responses } \\
\text { to coherent optic flow visual motion } \\
\text { compared to random visual motion. }\end{array}$ & $\begin{array}{l}\text { Study investigating the neural responses to the perception } \\
\text { of vection during viewing of visual optic flow stimuli. } \\
\text { pVIP showed higher activation when subjects reported } \\
\text { perceiving vection as compared to when they did not. }\end{array}$ \\
\hline & 12 & $\begin{array}{l}\text { Wall and Smith, } \\
(2008)\end{array}$ & $\begin{array}{l}\text { *Left: }-26,-56,43 \\
\text { *Right: } 25,-51,43\end{array}$ & $\begin{array}{l}\text { Higher responses to egomotion- } \\
\text { consistent visual motion compared to } \\
\text { egomotion- inconsistent, but coherent, } \\
\text { visual motion. }\end{array}$ & $\begin{array}{l}\text { Study investigating responses to egomotion-consistent } \\
\text { visual motion compared to egomotion- inconsistent, but } \\
\text { coherent, visual motion. }\end{array}$ \\
\hline & 13 & Zajac et al., (2019) & Right: $18,-62,60$ & $\begin{array}{l}\text { Higher responses to coherent visual } \\
\text { motion compared to scrambled visual } \\
\text { motion. }\end{array}$ & $\begin{array}{l}\text { Study investigating the connectivity between optic flow } \\
\text { responsive regions and the retrosplenial cortex and } \\
\text { hippocampus when subjects performed visual path } \\
\text { integration and turn counting tasks. There was an inverse } \\
\text { relation between self-reported navigational ability and } \\
\text { functional connectivity strength between pVIP and the } \\
\text { retrosplenial cortex, intracalcarine cortex and lingual } \\
\text { gyrus during the turn counting task. }\end{array}$ \\
\hline & 14 & $\begin{array}{l}\text { Zajac and Killiany, } \\
(2021)\end{array}$ & $\begin{array}{l}\text { Left: }-22,-62,62 \\
\text { Right: } 22,-60,62\end{array}$ & $\begin{array}{l}\text { Higher responses to coherent visual } \\
\text { motion compared to scrambled visual } \\
\text { motion. }\end{array}$ & $\begin{array}{l}\text { Study investigating the responses during visual path } \\
\text { integration and turn counting tasks in optic-flow } \\
\text { responsive brain regions of young and aged adults. There } \\
\text { was that stronger activity in pVIP during the visual path } \\
\text { integration task was associated with greater visual path } \\
\text { integration accuracy in aged adults. }\end{array}$ \\
\hline
\end{tabular}




\begin{tabular}{|c|c|c|c|c|c|}
\hline & No & Study & pVIP MNI coordinates & Definition of pVIP & Study approach and VIP-related findings \\
\hline \multirow[b]{2}{*}{ 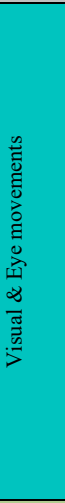 } & 15 & $\begin{array}{l}\text { Konen and Kastner, } \\
(2008 \mathrm{a})\end{array}$ & $\begin{array}{l}\text { *Left: }-35,-54,45 \\
\text { *Right: } 34,-55,46\end{array}$ & $\begin{array}{l}\text { Retinotopic mapping, where IPS5 was } \\
\text { defined as pVIP. }\end{array}$ & $\begin{array}{l}\text { The authors defined six topographically organized } \\
\text { posterior parietal regions (IPS1-5, \& SPL1) using a } \\
\text { memory-guided saccade paradigm. They then tested the } \\
\text { responses of these regions to eye movements (saccades } \\
\text { and smooth pursuit) and optic flow motion stimuli. IPS5 } \\
\text { (pVIP) responded to smooth pursuit eye movements and } \\
\text { optic flow patterns. }\end{array}$ \\
\hline & 16 & Konen et al., (2005) & $\begin{array}{l}\text { 1. optokinetic nystagmus } \\
(\text { OKN) > baseline } \\
\text { *Left: }-29,-50,44 \\
\text { *Right: } 27,-47,35 \\
\text { 2. smooth pursuit eye } \\
\text { movements (SPEM) }> \\
\text { baseline } \\
\text { *Left: }-36,-47,37 \\
\text { *Right: } 29,-50,36 \\
\text { 3. unpredictable SPEM > } \\
\text { baseline } \\
\text { *Right: } 37,-51,40\end{array}$ & $\begin{array}{l}\text { Functional responses in the } \\
\text { intraparietal sulcus to eye movements } \\
\text { compared to baseline, in whole-brain } \\
\text { analyses. }\end{array}$ & $\begin{array}{l}\text { Study investigating the neural responses to optokinetic } \\
\text { nystagmus (OKN) and smooth pursuit eye movements } \\
\text { (SPEM). pVIP activation was evident in contrasts } \\
\text { comparing OKN and SPEM to baseline fixation } \\
\text { conditions. }\end{array}$ \\
\hline 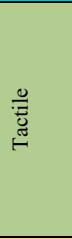 & 17 & $\begin{array}{l}\text { Huang and Sereno, } \\
\text { (2007) }\end{array}$ & $\begin{array}{l}\text { Coordinates were given } \\
\text { separately for face and } \\
\text { lip tactile activations for } \\
\text { one subject. pVIP } \\
\text { coordinates below are } \\
\text { the average of these two } \\
\text { sets of coordinates. } \\
\text { *Left: }-26,-45,54 \\
\text { *Right: } 31,-57,68 \\
\end{array}$ & $\begin{array}{l}\text { Topographic mapping of tactile stimuli } \\
\text { on the face. }\end{array}$ & $\begin{array}{l}\text { Study demonstrating the use of an MR-compatible system } \\
\text { to deliver air puff stimuli to multiple points on the face and } \\
\text { fingers. The region that responded to tactile stimuli on the } \\
\text { face was suggested as pVIP. }\end{array}$ \\
\hline 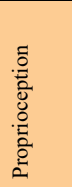 & 18 & Fasold et al., (2008) & $\begin{array}{l}\text { *Left: }-32,-48,43 \\
\text { *Right: } 40,-42,51\end{array}$ & $\begin{array}{l}\text { Higher responses to neck muscle } \\
\text { stimulation than superficial skin } \\
\text { vibration control stimulus. }\end{array}$ & $\begin{array}{l}\text { Study investigating neural responses during neck muscle } \\
\text { stimulation, which was used to simulate proprioceptive } \\
\text { input during changes in head position. }\end{array}$ \\
\hline \multirow{3}{*}{ 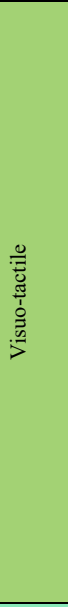 } & 19 & Cardini et al., (2011) & $\begin{array}{l}\text { Left: }-40,-36,36 \\
\text { Right: } 32,-52,44\end{array}$ & $\begin{array}{l}\text { Higher activity to any of the } 6 \\
\text { experimental conditions of a } 3 \\
\text { (viewing own face, other person's face } \\
\text { or object) } x 2 \text { (viewing a touch, paired } \\
\text { with a tactile stimulus on the subject's } \\
\text { face vs. viewing a hand approaching, } \\
\text { but not touching, and no tactile } \\
\text { stimulus) factorial design, compared to } \\
\text { a fixation baseline. }\end{array}$ & $\begin{array}{l}\text { Study investigating the integration of viewing touches to } \\
\text { the face with tactile stimuli on the subject's face. pVIP } \\
\text { showed higher activity when subjects viewed a touch and } \\
\text { received a tactile stimulus compared to when they did not } \\
\text { view a touch or receive a tactile stimulus. }\end{array}$ \\
\hline & 20 & Huang et al., (2017) & $\begin{array}{l}\text { Mean coordinates across } \\
\text { subjects for experiment } 1 \\
\text { (tactile only): } \\
\text { *Left: }-24,-43,58 \\
\text { *Right: } 27,-44,64 \\
\\
\text { Mean coordinates across } \\
\text { subjects for experiment } 2 \\
\text { (tactile and visual): } \\
\text { *Right: } 28,-44,69\end{array}$ & $\begin{array}{l}\text { Topographic mapping of tactile stimuli } \\
\text { on the face (experiment 1) and both } \\
\text { topographic mapping of tactile stimuli } \\
\text { on the face and looming visual stimuli } \\
\text { close to the face (experiment } 2 \text { ). }\end{array}$ & $\begin{array}{l}\text { Study that topographically mapped tactile stimuli around } \\
\text { the face and visual stimuli close to the face, using high- } \\
\text { resolution imaging. }\end{array}$ \\
\hline & 21 & $\begin{array}{l}\text { Sereno and Huang, } \\
\text { (2006) }\end{array}$ & $\begin{array}{l}\text { *Left: }-28,-50,64 \\
\text { *Right: } 29,-48,66\end{array}$ & $\begin{array}{l}\text { Topographic mapping of tactile stimuli } \\
\text { on the face and visual stimuli close to } \\
\text { the face. }\end{array}$ & Topographic mapping study. \\
\hline \multirow{3}{*}{ 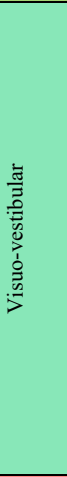 } & 22 & $\begin{array}{l}\text { Aedo-Jury et al., } \\
(2020)\end{array}$ & $\begin{array}{l}\text { *Left: }-45,-43,40 \\
\text { *Right: } 40,-51,43\end{array}$ & $\begin{array}{l}\text { Functional localizer, higher responses } \\
\text { to egomotion-consistent visual motion } \\
\text { compared to egomotion- inconsistent, } \\
\text { but coherent, visual motion (Wall and } \\
\text { Smith, 2008). }\end{array}$ & $\begin{array}{l}\text { Study investigating responses to vestibular stimulation in } \\
\text { brain regions that respond to egomotion-consistent visual } \\
\text { stimuli. pVIP responded to antero-posterior galvanic } \\
\text { vestibular stimulation (GVS). }\end{array}$ \\
\hline & 23 & $\begin{array}{l}\text { Billington } \quad \text { and } \\
\text { Smith, (2015) }\end{array}$ & Coordinates not stated. & $\begin{array}{l}\text { Functional localizer, higher responses } \\
\text { to egomotion-consistent visual motion } \\
\text { compared to egomotion- inconsistent, } \\
\text { but coherent, visual motion (Wall and } \\
\text { Smith, 2008). }\end{array}$ & $\begin{array}{l}\text { Study investigating neural responses to visual motion and } \\
\text { artificial vestibular stimulation based on visual } \\
\text { stimulations emulating natural head roll. The phase of } \\
\text { visual and vestibular motion could be decoded from pVIP. }\end{array}$ \\
\hline & 24 & $\begin{array}{ll}\text { Schindler } & \text { and } \\
\text { Bartels, (2018) } & \end{array}$ & Coordinates not stated. & $\begin{array}{l}\text { Functional localizer, comparing } \\
\text { coherent vs. random visual motion } \\
\text { (Wall and Smith, 2008). In addition, a } \\
\text { dorsal pVIP was identified in a whole } \\
\text { brain analysis by its responses to } \\
\text { congruent visual flow and head } \\
\text { motion. }\end{array}$ & $\begin{array}{l}\text { Study investigating responses to natural head motion } \\
\text { paired with congruent or incongruent visual flow stimuli. } \\
\text { pVIP defined as a functional ROI did not show a } \\
\text { difference in responses to congruent and incongruent } \\
\text { conditions but did show higher activation to head } \\
\text { movement compared to when the head was static. A whole } \\
\text { brain analysis comparing congruent and incongruent } \\
\text { conditions identified activity in pVIP. }\end{array}$ \\
\hline 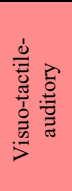 & 25 & $\begin{array}{l}\text { Bremmer et al., } \\
(2001)\end{array}$ & $\begin{array}{l}\text { *Left: }-40,-43,45 \\
\text { *Right: } 37,-48,50\end{array}$ & $\begin{array}{l}\text { Conjunction of higher responses to } \\
\text { visual, tactile, and auditory motion } \\
\text { stimuli compared to static control } \\
\text { stimuli. }\end{array}$ & $\begin{array}{l}\text { Study investigating the conjunction of multisensory } \\
\text { responses. For visual stimulation, coherent moving dots } \\
\text { were compared to static random dots. For tactile } \\
\text { stimulation, air flow over the forehead was compared to } \\
\text { no tactile stimulation. For auditory stimulation, auditory } \\
\text { stimuli producing an illusion of sound motion was } \\
\text { compared to no auditory stimuli except for scanner noise. }\end{array}$ \\
\hline
\end{tabular}




\begin{tabular}{|c|c|c|c|c|c|}
\hline & No & Study & pVIP MNI coordinates & Definition of pVIP & Study approach and VIP-related findings \\
\hline 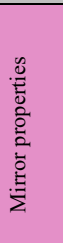 & 26 & Ferri et al., (2015) & Left: $-30,-52,62$ & $\begin{array}{l}\mathrm{pVIP} \text { was termed a dorsal part of the } \\
\text { dorsal intraparietal sulcus anterior. } \\
\text { Stronger responses to interpersonal } \\
\text { actions compared to object-related and } \\
\text { skin-displacing actions, and its } \\
\text { anatomical location close to previously } \\
\text { proposed coordinates for pVIP (Cardin } \\
\text { and Smith, 2010; Sereno and Huang, } \\
\text { 2006). }\end{array}$ & $\begin{array}{l}\text { Study investigating responses to observation of upper- } \\
\text { limb actions with different motor goals (object-related, } \\
\text { skin-displacing and interpersonal). pVIP showed higher } \\
\text { responses to interpersonal actions as compared to object- } \\
\text { related and skin-displacing actions. }\end{array}$ \\
\hline 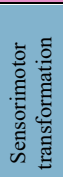 & 27 & Serra et al., (2019) & $\begin{array}{l}\text { Left: }-25,-57,56 \\
\text { Right: } 29,-52,55\end{array}$ & $\begin{array}{l}\text { Higher responses to coherent than } \\
\text { random visual motion (Cardin and } \\
\text { Smith, 2010). }\end{array}$ & $\begin{array}{l}\text { This study investigated responses to leg movements } \\
\text { similar to those made during walking in brain regions that } \\
\text { responded to egomotion-consistent visual motion. pVIP } \\
\text { did not show responses to leg movements. }\end{array}$ \\
\hline 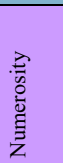 & 28 & Eger et al., (2015) & $\begin{array}{l}\text { Left: }-33,-49,57 \\
\text { Right: } 29,-49,54\end{array}$ & $\begin{array}{l}\text { Functional localizer, conjunction of } \\
\text { higher responses to tactile stimulation } \\
\text { on the face vs. static dots baseline, and } \\
\text { higher responses to } \\
\text { expanding/receding visual dots motion } \\
\text { vs. static dots baseline. }\end{array}$ & $\begin{array}{l}\text { Study investigating the neural coding of numerosity. The } \\
\text { numerosity of sets of dots stimuli could be decoded from } \\
\text { pVIP and could be cross-decoded across different visual } \\
\text { field locations. }\end{array}$ \\
\hline
\end{tabular}

The distinction of active and passive situations is critical for perceiving motion: when movement occurs while the animal is passive, the movement usually signals that impact from an external object is imminent. Accordingly, animals usually respond with fast, reflexive defensive movements. In contrast, such reflexive responses must often be suppressed when the perceived movement originates from the animal's own movement, rather than from that of an external threat. Therefore, the modulatory role of active movement, such as smooth pursuit, indicates that macaque VIP may be central to sensory-motor transformation and decision-making and, accordingly, be at the intersection of perception and action - a role often assigned to PPC also more globally (Batista et al., 1999, Medendorp and Heed, 2019; Zhang and Barash, 2000). We will return to this notion again later in Section 2.3.

In humans, area pVIP\#1 separates between egomotion and nonegomotion optic flow patterns, evident in fMRI activation differences between the two contexts (Wall and Smith, 2008). Furthermore pVIP\#2 dissociates object-motion from egomotion (Field et al., 2020). As in the macaque, pVIP (defined using approach \#1, but exact coordinates not specified) encoded changes in heading direction from visual flow cues (Furlan et al., 2014).

In natural situations, visual egomotion cues usually coincide with vestibular cues. When human participants view isolated visual optic flow cues in experiments, they often report experiencing vection. Many people know the perceptual experience of vection from an illusion that occurs when looking out of a standing train onto a moving one: it feels as if it is the own train, rather than the other, that is moving. Vection, thus, is the experience of self-motion induced purely visually. pVIP\#1 is one of several areas that were consistently modulated by experiencing vection (Uesaki and Ashida, 2015).

The relevance of active vs. passive egomotion has been addressed with fMRI for human pVIP\#3. Participants visually experienced heading changes in a virtual environment in which they were either passively moved or, instead actively navigated (though virtually, Huang et al., 2015). The two conditions did not result in any significant activation differences. Discrepancies between the two species, such as those discussed last, may reflect methodological differences between studies, or they may indicate that $\mathrm{pVIP} \# 3$ does not process egomotion. Active vs. passive egomotion has not been explicitly tested in $\mathrm{pVIP} \# 1 / \# 2$, and so this particular example serves to illustrate one difficulty that we deem critical in understanding VIP. every human study discussed here targeted a human VIP homologue based on one of several functional or anatomical definitions. Accordingly, we have learned bits and pieces about each of the three areas of the pVIP complex but cannot truly infer functional homology for any of them.

In macaques, the two core visual properties of near-space and large field flow field coding partially co-localize within the macaque IPS, as assessed with macaque fMRI (Cléry et al., 2015b). A part of the macaque IPS fundus preferentially coded for visual stimuli near the face (Cléry et al., 2018). This region was fully encompassed by a large activation along the IPS fundus in response to large field optic flow and translational stimuli (Guipponi et al., 2013). Whether individual single neurons partake in both visual functions has not been tested.

In contrast, near-face and optic flow stimulation evoked peak activations at distinct parietal locations in humans. Near-face activations were located just dorsal to the IPS in the superior parietal cortex (pVIP\#3, see Fig. 2A, near-face visual stimuli Sereno and Huang, 2006: purple spheres, number 21). Most egomotion-related activations were located within the IPS, in the more posterior pVIP\#1 (see Fig. 2A, Cardin and Smith, 2010; Di Marco et al., 2021; Serra et al., 2019; Uesaki and Ashida, 2015; Wall and Smith, 2008; Zajac et al., 2019; Zajac and Killiany, 2021: orange spheres, numbers 2, 3, $11,12,13,14 \& 27$ ), with the exception of one study in which optic flow clustered together with near-face activations (Huang et al., 2015: purple sphere, number 6).

Viewed from a higher perspective, how might heading and selfmotion perception relate to a generalized functional role of VIP? Both functions imply that movement consequences of the own body and of objects in the environment must be predicted in space and time. Thus, VIP may compute predictions and/or prediction errors. Indeed, macaque VIP exhibits anticipatory (i.e., predictive) responses when an expected self-motion stimulus is delayed (Churan et al., 2021) and when true self-motion direction is different from that predicted by visual cues (Schmitt et al., 2021). Such predictive processing is likely to contribute to very fast responses to unexpected sensory input. 
Table 2. Comparison of the functional properties that have been investigated in VIP. The first column refers to the review section in which each function is discussed in depth. The second column shows the eight functional properties discussed in section 2. The background coloring matches that of Figure 4, in which we present the functional networks VIP is a part of, and that broadly match the functions listed here. For each function, we list several subtopics with the experimental methods used in the respective studies. For monkeys, the most commonly used methods are single-unit recordings (neuronal) and functional magnetic resonance imaging (fMRI), but microstimulation (MS) and inactivation (In) have also been applied. For humans, the most commonly used method is fMRI, including meta-analysis of fMRI data (MA), but transcranial magnetic stimulation (TMS) and positron emission tomography (PET) have also been applied.

\begin{tabular}{|c|c|c|c|c|c|c|c|}
\hline & \multirow{2}{*}{\multicolumn{3}{|c|}{$\begin{array}{l}\text { Species } \\
\text { Recording method }\end{array}$}} & \multicolumn{2}{|l|}{ Monkey } & \multicolumn{2}{|c|}{ Human } \\
\hline & & & & \multirow{2}{*}{$\begin{array}{l}\text { Single neurons } \\
\mathrm{x}\end{array}$} & \multirow[t]{2}{*}{ fMRI } & \multirow[t]{2}{*}{ fMRI } & \multirow[t]{2}{*}{ others } \\
\hline \multirow{16}{*}{ 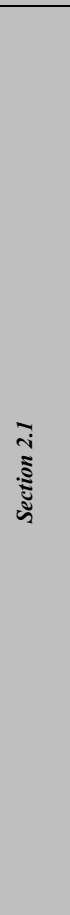 } & \multirow{5}{*}{$\stackrel{5}{5}$} & \multicolumn{2}{|c|}{ Moving stimulus (mapping of RFs) } & & & & \\
\hline & & \multicolumn{2}{|c|}{ Near vs. far visual space } & $\mathrm{x}$ & $\mathrm{x}$ & $\mathrm{x}$ & \\
\hline & & \multicolumn{2}{|l|}{ Heading perception } & $\mathrm{x}, \mathrm{MS}$ & & $\mathrm{x}$ & \\
\hline & & \multirow[t]{2}{*}{ Egomotion } & Egomotion vs. non-egomotion & & $\mathrm{x}$ & $\mathrm{x}$ & \\
\hline & & & Egomotion vs. object motion & & & $\mathrm{x}$ & \\
\hline & \multirow{3}{*}{ 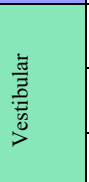 } & \multicolumn{2}{|l|}{ Tuning to head velocity } & $\mathrm{x}$ & & $\mathrm{x}$ & \\
\hline & & \multicolumn{2}{|c|}{ Body- and world-centered reference frames } & $\mathrm{x}$ & & & \\
\hline & & \multicolumn{2}{|c|}{ Passive vs. active movement } & $\mathrm{x}$ & & & \\
\hline & ప & \multicolumn{2}{|c|}{ Eye proprioceptive signals } & $\mathrm{x}$ & & $\mathrm{x}$ & \\
\hline & 感 & \multicolumn{2}{|c|}{ Neck proprioceptive signals } & & & $\mathrm{x}$ & \\
\hline & & \multicolumn{2}{|l|}{ Mapping of RFs } & $\mathrm{x}$ & $\mathrm{x}$ & $\mathrm{x}$ & \\
\hline & $\stackrel{\varrho}{\tilde{E}}$ & \multicolumn{2}{|c|}{ Alignment of visual and tactile RFs } & $\mathrm{x}$ & & $\mathrm{x}$ & \\
\hline & & \multicolumn{2}{|c|}{ Face dorsal and ventral subdivisions } & & & $\mathrm{x}$ & \\
\hline & & \multicolumn{2}{|l|}{ Basic responses } & $\mathrm{x}$ & $\mathrm{x}$ & & \\
\hline & : & \multicolumn{2}{|l|}{ Sequence coding } & & $\mathrm{x}$ & $\mathrm{x}$ & \\
\hline & & \multicolumn{2}{|l|}{ Vocalization } & & $\mathrm{x}$ & & \\
\hline \multirow{11}{*}{ 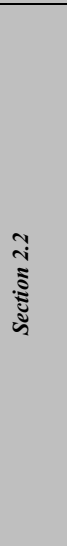 } & & Integration mechanisı & & $\mathrm{x}$ & & $\mathrm{x}$ & TMS \\
\hline & & Spatial alignment and & eference frames across modalities & $\mathrm{x}$ & & & \\
\hline & & Convergence of visua & tactile, and auditory modalities in IPS & & $\mathrm{x}$ & $\mathrm{x}$ & \\
\hline & & Face/head & Face vs. periphery & & $\mathrm{x}$ & $\mathrm{x}$ & \\
\hline & 岕 & & Visual-tactile interaction & & $\mathrm{x}$ & $\mathrm{x}$ & \\
\hline & 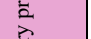 & & Far vs. near space & $\mathrm{x}$ & $\mathrm{x}$ & $\mathrm{x}$ & PET, TMS \\
\hline & : & & Topographic mapping & & & $\mathrm{x}$ & \\
\hline & $\stackrel{ \pm}{\Xi}$ & Hand peripersonal sp. & & & & $\mathrm{x}$ & TMS \\
\hline & & Egomotion & Visual-vestibular integration & $\mathrm{x}$ & & $\mathrm{x}$ & \\
\hline & & & Interference study & $\mathrm{x}, \mathrm{In}$ & & & \\
\hline & & & Perception and choice signal & $\mathrm{x}$ & & & \\
\hline & & Oculomotor & Generation of eye movements & & $\mathrm{x}$ & $\mathrm{x}$ & \\
\hline$\stackrel{n}{\Xi}$ & 䒕 & & $\begin{array}{l}\text { Mechanism of maintaining stability } \\
\text { during eye movements }\end{array}$ & $\mathrm{x}$ & & & \\
\hline 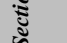 & 定 & Whole body motor & Defensive behaviors & MS & & & \\
\hline & & & Reaching/feeding & & & $\mathrm{x}$ & \\
\hline 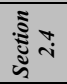 & 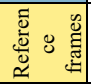 & Eye, head and/or body & centered reference frames & $\mathrm{x}$ & & $\mathrm{x}$ & TMS \\
\hline & $\underline{\tilde{g}}$ & Numerosity & & $\mathrm{x}$ & & $\mathrm{x}$ & \\
\hline$\hat{i}$ & 苞 & Sequence processing & & $\mathrm{x}$ & $\mathrm{x}$ & $\mathrm{x}$ & \\
\hline 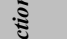 & $\stackrel{\Xi}{\Xi}$ & Working memory & & $\mathrm{x}$ & & $\mathrm{x}$ & \\
\hline n & है & Embodiment and own & ship & & & MA & \\
\hline
\end{tabular}

VIP belongs to a motion detection network (Figure 4). The "motion detection" VIP network starts with V2 (Figure 4A, 4B), the earlies area in which motion direction can be decoded (Levitt et al., 1994).
V2, V3, and MT are considered early or intermediate visual areas; they feature small- to medium-sized RFs and relatively simple, linear directional preference (Albright, 1984; Gattass et al., 1988). Another 
highly direction-selective area in the network is V6 (Galletti et al., 1991), which is connected to most areas in the network (Galletti et al., 2001). VIP and MST can be considered higher order areas and process more complex information of whole-field optic flow, such as translational motion and rotation (Duffy and Wurtz, 1991; Gu et al., 2006). In computational modelling of visual hierarchical organization, VIP and MST are consistently assigned the same hierarchical level (Hilgetag et al., 1996). Both areas project to area $7 \mathrm{a}$ and to superior temporal polysensory area (STP), the roles of which within the motion detection network are yet to be discovered. Despite their similarities, several aspects differentiate VIP from MST (Britten, 2008). First, the over-representation of central near visual space and, accordingly, a role in near-field motion analysis is unique to VIP. Second, greater attentional effects in VIP than MST for motion detection suggest that VIP is more closely associated with behavior (Cook and Maunsell, 2002a, 2002b) - consistent with our conclusion of a dedicated sensorimotor role for VIP that is derived from its characteristics in egomotion processing. Finally, eye position modulates VIP RFs, and VIP neurons' reference frames are often intermediate between eye- and head-centered, whereas neurons in MST exhibit strictly eye-centered visual coding (Avillac et al., 2007, 2005; Duhamel et al., 1997).

\subsubsection{Vestibular system}

VIP encodes head velocity. In ecological conditions, egomotion perception relies on a combination of low-level visual, vestibular, and proprioceptive sensory cues. In macaque VIP, about a third of neurons respond to horizontal rotation (Bremmer et al., 2002b) These vestibular responses are typically in phase with head velocity, and sometimes signal head acceleration or head position (Klam and Graf, 2003). Notably, neurons' preferred response direction during horizontal head rotations differs depending on whether the monkey actively turns its head or the head is turned passively (Klam and Graf, 2006). This difference between active vs. passive conditions is reminiscent of the visual egomotion coding discussed above.

Reference frames in VIP vary across neurons. The preferred vestibular heading direction of single VIP neurons is independent of eye and head position (Chen et al., 2013). Moreover, when gaze is fixed relative to the body, VIP vestibular heading tuning is egocentric and body-centered. In contrast, when gaze is fixed relative to the world, tuning is intermediate between body- and world-centered reference frames (Chen et al., 2018), though the experimental procedures of this experiment did not further distinguish between body-centered and head-centered frames of reference. VIP's vestibular coding contrasts with the eye to head-centered reference frame coding it expresses for vision (Duhamel et al., 1997). However, VIP neurons do also encode eye and head position for vestibular signals by way of gain fields, that is, position-dependent modulation of their firing rate. Thus, vestibular and visual VIP responses reflect eye and head position through different mechanisms.

VIP belongs to a vestibular network. The core areas of vestibular processing (Figure 4A, 4C) are the parieto-insular vestibular cortex (PIVC, overlapping with retro-insular area Ri in Lewis and Van Essen, 2000a), areas 2V, 3a (Gu, 2018; Guldin and Grüsser, 1998), and 7a (Avila et al., 2019). MST (Gu et al., 2007; Ugolini et al., 2019), FEF (Gu et al., 2016) and potentially ventral LIP (Ugolini et al, 2019) are also part of the vestibular network. The flow of information across the vestibular system is currently unknown, although neuronal response latencies suggest a hierarchy from PIVC to VIP to MSTd, with the shortest vestibular responses in PIVC (Chen et al., 2011).

Location of human IPS vestibular activations. Investigating vestibular stimulation is challenging in the MR environment, with the head highly constrained in the scanner's head coil. One study analyzed fMRI responses shortly after participants made head movements, with the head stabilized, benefitting from the slow dynamics of the BOLD signal (Schindler and Bartels, 2018). The study identified pVIP with approach \#1 and reported its activation by active head motion. Another study applied binaural monopolar galvanic vestibular stimulation (GVS), that is, application of small electric currents to the ears' vestibular nerves (Aedo-Jury et al., 2020). This study identified pVIP with approach \#1, but the resulting activation appears to have been located closer to our $\mathrm{pVIP} \# 2$ cluster (see Figure 3). GVS that evokes antero-posterior vestibular sensations activated pVIP, but GVS that evokes lateral sensations did not. Note the contrast with macaque single neuron preferences for lateral signals during true translational and rotational head movements (Chen et al., 2011).

\subsubsection{Proprioception}

Proprioception is important for dynamic tracking of gaze information: together with other sensory input, proprioception contributes to encoding eye position in the head and head position relative to the trunk. The combination of eye position with visual (retinal) information allows VIP to derive head-centered visual representations, both in single cells and at the population level (Bremmer et al., 2002a; Duhamel et al., 1997).

Eye proprioceptive signals. VIP neurons' responses to optic flow are gain-modulated by eye position, independently of whether the animal is currently planning an eye movement or not (Bremmer et al., 2002a; Duhamel et al., 1997). During planning, eye position may be derived from an efference copy, but in other cases eye position must be determined from proprioception. It has been suggested (but never actually demonstrated) that such proprioceptive eye position information originates from the eye region of primary somatosensory cortex and represents eye position in the orbit (Wang et al., 2007). However, in the time around an eye movement, final eye position is decodable from VIP neurons both before the movement as well as after (Morris et al., 2016, 2013, 2012). At that time, proprioception cannot have signaled the final eye position that would result from the impending saccade. This result, therefore, argues for the relevance of the efference copy in eye position information. Yet, this conjecture remains to be confirmed experimentally.

Eye position coding has been related to pVIP only indirectly in humans. One study characterized saccade-related topographic maps along a large portion of PPC including the entire pVIP complex. Participants performed memory-guided saccades from varying starting points relative to the head/torso to targets at eccentric locations around the visual field (Connolly et al., 2015). Topographic maps showed subtle changes as a function of gaze start point throughout the PPC, leading the authors to suggest that they were dominated by head- or body-centered coding.

Neck proprioceptive signals. Proprioceptive input from neck muscle spindle receptors contributes to the perception of how the head is positioned on the torso. Real head motion elicits both proprioceptive and vestibular signals concurrently, so a separation of the two types of information in neural coding is not possible under natural conditions. To our knowledge, neck proprioceptive responses have not been assessed in macaque VIP. However, neck velocity signals have been described in a subregion of the FEF responsible for smooth pursuit, using direct muscle spindle stimulation (Fukushima et al., 2010). This region is connected with area VIP (Lewis and Van Essen, 2000 b), suggesting neck velocity signals may also exist in VIP.

Isolating proprioceptive head movement information has been achieved in humans by applying vibration to the posterior neck muscles while the head remains fixed (Goodwin et al., 1972). This manipulation creates a continuous kinesthetic illusion of head tilt and head rotation, along with corresponding illusory visual motion. It evoked fMRI activation close to the junction of the IPS and the postcentral sulcus (PCS), a location within pVIP\#2 (Fasold et al., 2008; see Fig. 3). In contrast, no IPS activation was reported during static variations of head to trunk angle (Paschke et al., 2019).

The VIP proprioceptive network. The core cortical proprioceptive regions are the primary somatosensory areas $3 \mathrm{a}, 3 \mathrm{~b}, 1,2$, and 5 (Padberg et al., 2009; see Figure 4A, 4C). The proprioceptive VIP network extends to PIVC (Grüsser et al., 1990), LIP (Snyder et al., 1998), primary motor cortex (M1) (Hatsopoulos and Suminski, 2011), F4 in the PMv (Graziano et al., 1997a; Graziano, 1999) and the FEF (Sun and Goldberg, 2016; Thura et al., 2008). This network appears to be specialized for processing head and neck proprioception information for eye movements, possibly to maintain a visually stable image during movement (Grüsser et al., 1990; Snyder et al., 1998; Sun and Goldberg, 2016). 
A

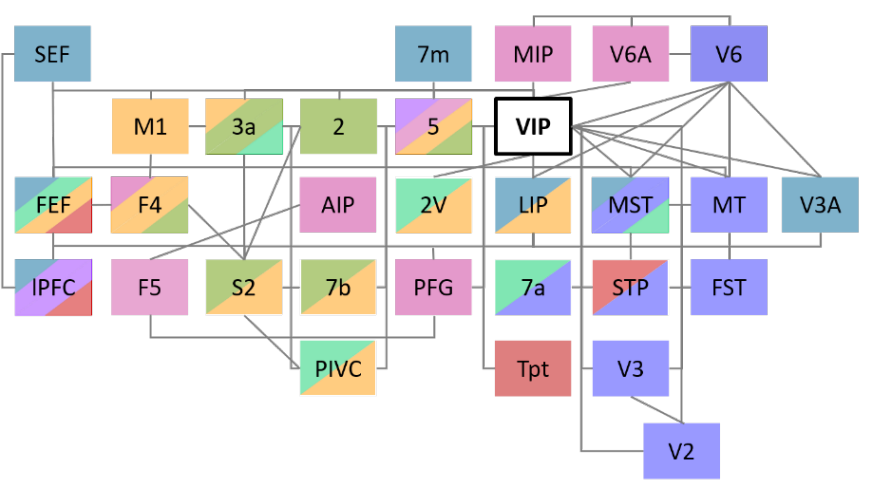

Motion detection
Eye movement
Vestibular
Tactile
Proprioception
Auditory
Peripersonal space

Numerosity-working memory

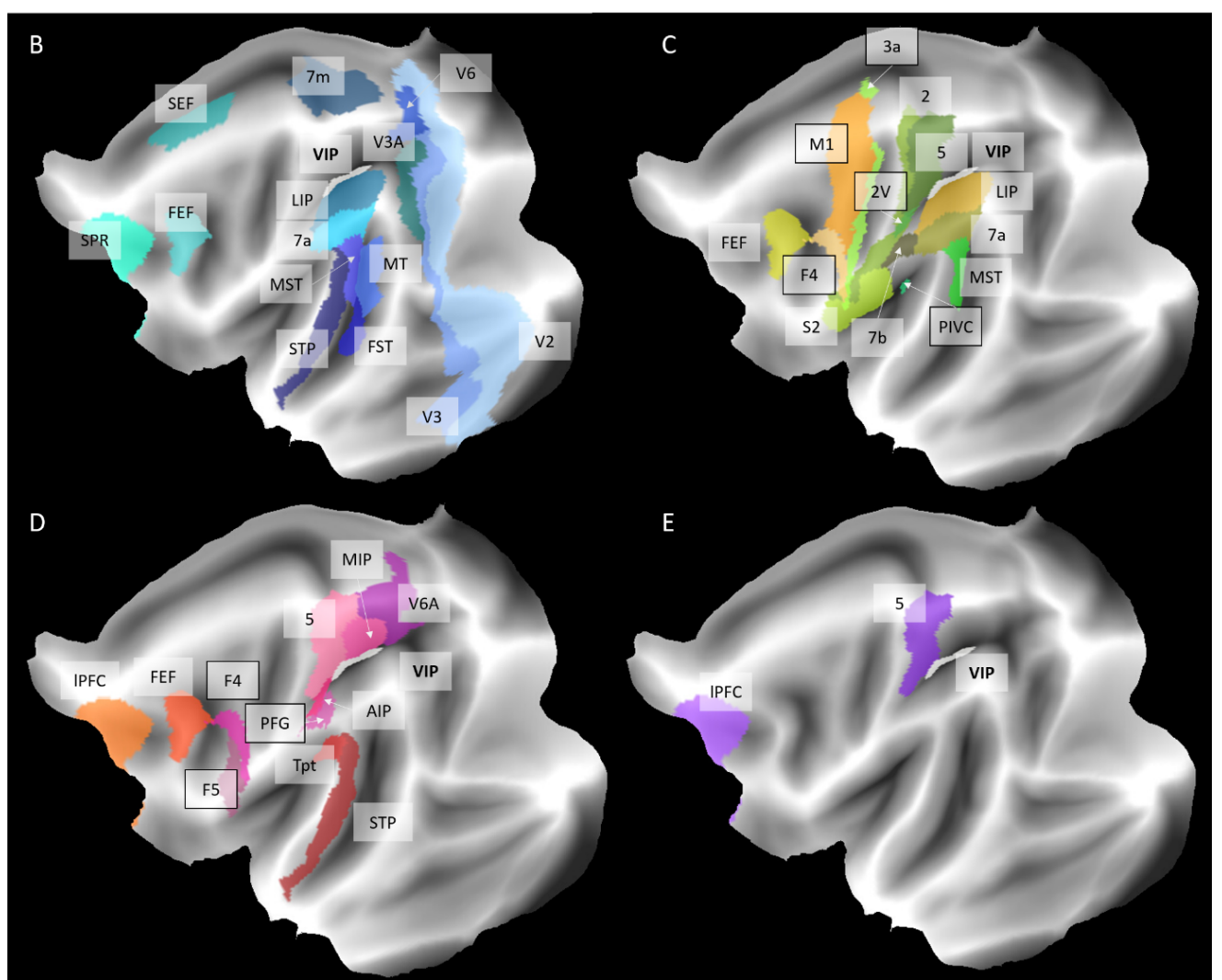

Figure 4. Selected anatomical links highlighting eight of the different functional aspects associated with VIP that are described in section 2. (A) Areas directly linked to VIP, arranged by relative anatomical location. Areas are colored according to eight functional categories: viul motion, eye movement, vestibular, tactile, proprioception, auditory, peripersonal space and numerosity/working memory. Striped background colors show the overlap of multiple functional categories in one area. (B) Visual motion and eye movement areas displayed on a flat map. (C) Vestibular, tactile and memory. Striped background colors show the overlap of multiple functional categories in one area. (B) Visual motion and eye movement areas displayed on a flat map. (C) Vestibular, tactile and
proprioceptive areas. (D) Auditory and peripersonal space areas. (E) Numerosity/working memory areas. (B-E) Areas are colored according to the functional category color in (A). Areas without an outline are from the Markov et al. atlas (2014) and areas with a black outline are from the Paxinos et al. atlas (1999). PFG is the rostral part of 7a. 7m: medial part of area 7, AIP: anterior intraparietal area, FEF: frontal eye field, FST: fundus of the superior temporal sulcus area, LIP: lateral intraparietal area, IPFC: lateral prefrontal cortex, M1: primary motor cortex, MIP: medial intraparietal area, MST: medial superior temporal area, MT: middle temporal area, PIVC: parieto-insular vestibular cortex, S2: second somatosensory area, SEF: supplementary eye field, STP: superior temporal polysensory area, Tpt: temporoparietal area, V2: second visual area, V3: third visual area, VIP: ventral intraparietal area.

\subsubsection{Tactile \& somatosensory sensation}

About 70\% of VIP neurons respond to touch (Duhamel et al., 1998).

Notably, all tactually responsive neurons also respond to visual stimulation, whereas the reverse is not true and not all visual neurons exhibit tactile responses. Tactile and visual RFs are aligned when gaze is straight-ahead and exhibit comparable spatial characteristics across the two modalities (Avillac et al., 2005; Duhamel et al., 1998). For example, small tactile RFs on the muzzle are paired with small foveal visual RFs, and larger tactile RFs on the side of the head and body coincide with larger peripheral visual RFs.

Notably, however, $\sim 85 \%$ of VIP RFs are on the head (Duhamel et al., 1998), and macaque fMRI experiments have confirmed this somatosensory over-representation of the head (Wardak et al., 2016). Lips and mouth cover a large area in the primate homunculus, and one might consider this region a tactile fovea. A fovea-like, tactile role of the snout fits well with VIP's focus on near-central visual space close to the eyes and head. Similarly, VIP's responses to optic flow heading cues predict impact to the head and face, again fitting well with the detailed tactile face representation. Notably, tactile fMRI activation was restricted to small portions of the anatomically defined macaque VIP, suggesting that VIP contains (modality-)specialized subregions (Guipponi et al., 2013) - a point we will return to in Section 3.

The matched tactile-visual properties are a unique feature of macaque VIP, compared to the rest of PPC. Therefore, several attempts to characterize human pVIP using fMRI have focused on this feature. One study located pVIP\#2 in the fundus of the human IPS based on the conjunction of activation to tactile, auditory, and visual motion (Bremmer et al., 2001; see Fig. 2). PVIP\#2 was also 
activated by large field visual egomotion flow-fields (Field et al., 2020), suggesting a close functional match with macaque VIP. Yet, another set of studies has located pVIP\#3 at the intersection between the IPS and the post-central gyrus based on fMRI high resolution mapping of face somatosensory responses, as well as high resolution mapping of near-face visual space (Huang and Sereno, 2007; Sereno and Huang, 2014, 2006; see Fig. 2). In fact, tactile and visual topographic maps were roughly aligned. This result suggests that neurons in this human PPC area show similar, matched visuo-tactile RFs as those of macaques, even if the area-wide macroorganizational overlap in fMRI cannot provide conclusive evidence about single neuron RF properties.

From a connectivity perspective, the tactile and proprioceptive cortical networks are quite intermingled because their respective thalamic projections to cortex are highly convergent. Indeed, both ventroposterior nucleus - the thalamus relay for cutaneous inputs and ventroposterior superior nucleus - the thalamus relay for proprioceptive inputs - project to areas $3 \mathrm{a}, 3 \mathrm{~b}, 1,2$, and 5 , albeit with different strength (Padberg et al., 2009). The core cortical tactile and proprioceptive regions are the primary somatosensory areas $3 \mathrm{a}, 3 \mathrm{~b}, 1$ and 2 in anterior parietal cortex. This core tactile/proprioceptive network then roughly divides into two somatosensory streams: a ventral stream that includes at its early stages the secondary somatosensory area (S2) and the parietal ventral area (PV) and has been linked to cognitive functions such as decision-making and attention, and a dorsal stream that includes at its early stages areas 5 and $7 \mathrm{~b}$ and has been linked to motor behaviors such as reaching and grasping (Delhaye et al., 2018). VIP receives direct projections from $\mathrm{S} 2$ and possibly from PV, as well as from areas 5 and $7 \mathrm{~b}$ ( Figure $4 \mathrm{~A}$, 4C, Lewis \& Van Essen, 2000a). It is thus connected with both somatosensory streams.

\subsubsection{Audition}

Basic auditory responses. Fewer studies have focused on the auditory modality than on touch and vision in VIP. Nonetheless, $80 \%$ percent of macaque VIP neurons responded to illusory motion created through binaural stimulation, demonstrating that VIP is a truly multisensory area. Reminiscent of the matching RFs in vision and touch, visual and auditory RFs also exhibited significant overlap (Schlack et al., 2005). Moreover, fMRI activations were maximal for complex auditory stimuli that produced a percept of coherent movement closely around the head (Guipponi et al., 2013) - a feature, thus, that we have encountered consistently for all modalities, and that suggests that the overarching function of VIP must be supramodal. Notably, however, auditory motion responses were restricted to small portions of the anatomically defined macaque VIP, suggesting that VIP contains (modality-)specialized subregions (Guipponi et al., 2013) - a point already mentioned for touch and which we will return to in Section 3.

In humans, illusory auditory motion created through binaural stimulation, similar to that used in some macaque experiments, elicited fMRI activation in pVIP\#2, and the activated region overlapped with (and was encompassed by) those responding to tactile air flow and visual flow fields (Bremmer et al., 2001). This study based its conclusion that pVIP\#2 was the homologue of macaque VIP on the tri-modal responsiveness of macaque VIP and the human PPC region identified here.

Auditory sequence coding. Although the VIP responses to auditory motion fit well with its response to touch and vision, macaque VIP auditory responses are not restricted to motion. For instance, fMRI activations were evident in VIP when macaques heard rare sounds that violated auditory sequences (Uhrig et al., 2014; Wang et al., 2015). The human brain also exhibits fMRI activation to such manipulations; in fact, these activations appear roughly compatible with putative $\mathrm{pVIP} \# 1$ in the left hemisphere and pVIP\#2 in the right hemisphere (Wang et al., 2015; see Fig. 3b). If the human brain processes auditory sequences in two pVIP clusters, the underlying functionality may in fact rely on combining several specialized core functions housed by VIP. However, hemispheric specialization has not received any attention for macaque or human VIP; moreover, recall that there were inconsistencies between the two hemispheres with respect to matching VIP to cytoarchitectonically defined regions. We will return to possible specializations of the different pVIP clusters in Section 3.
Vocalization. The fundus of macaque IPS is activated by complex sounds including own-species calls (Ortiz-Rios et al., 2015) and responds to human and monkey vocalizations (although not selectively, Joly et al., 2011, 2012). Anatomically, these macaque fMRI activations correspond to VIP. When social visual stimuli are presented together with vocalizations, the fundus of the IPS responds selectively to vocalizations that are congruent with the visual stimuli in meaning (Froesel et al., 2021). This finding opens the possibility that VIP is involved in highly complex social behavior based, in part, on its near-space spatial characteristics. This idea has already been proposed based on a very different research direction, namely VIP's role in eliciting defensive movements (Graziano and Cooke, 2006; see Section 2.3.2) and modulation of visuo-tactile processing in humans in collaborative task settings (Heed et al., 2010). To our knowledge, voice-associated responses have not been reported for human IPS. However, it is unclear whether this is a genuine functional difference between the two species, or whether such responses could be uncovered in humans by manipulating the proximity of stimuli to the body.

VIP is part of an auditory cortical network. Multiple areas within and around the auditory cortex link to VIP (Lewis and Van Essen, $2000 \mathrm{~b}$ ). However, there is no consensus about the organization of auditory cortex (Brewer and Barton, 2016), and this makes it difficult to match neurophysiological findings with tract-tracing connectivity studies. With this cautionary note in mind, VIP is strongly connected with the temporoparietal area (Tpt) in the "parabelt" of auditory cortex (Figure 4A, 4D, Lewis \& Van Essen, 2000a; Rauschecker, 2013). Tpt responds to pure auditory tones under anesthetized condition (Hikosaka et al., 1988) but to somatosensory and visual stimuli in awake monkeys (Leinonen et al., 1980), suggesting that the sensory convergence evident in VIP assembles across multiple areas. Auditory information reaches the prefrontal cortex, including FEF and area 46 of the lateral prefrontal cortex, through direct projections from VIP (Romanski et al., 1999). The selectivity of VIP for near-head auditory stimuli would place VIP in the auditory equivalent of the dorsal visual stream (Bizley and Cohen, 2013; Rauschecker, 2011). More research is needed here.

\subsection{Summary}

This section reviewed unimodal sensory responses of VIP. We would like to emphasize three points. First, most of the properties described in macaque VIP have been reproduced in human pVIP, but different properties usually activate one or two of the three distinct pVIP\#1/\#2/\#3. Second, these multiple sensory signals collectively coincide with the coding of body, or head, posture and movement relative to the (dynamic) environment, with a particular emphasis on the space close to the head (Cléry and Ben Hamed, 2018). These characteristics are classically interpreted as indicating a role of VIP in coding peripersonal space and egomotion. Third, the additional presence of voice-related responses as well as face mirroring properties (see below, Section 2.5.4), may point towards an involvement of VIP in social functions. In any case, the function of VIP does not reveal itself from any single one of the many relevant sensory cues independently, but rather from their combination and integration. We will discuss these aspects in detail next.

\subsection{Multisensory processing}

Section 2.1 has demonstrated a key property of VIP for both humans and monkeys: it receives information from multiple sensory modalities. Moreover, the orderly overlap of spatial RFs suggests that VIP purposely integrates information across modalities. From a neurophysiological point of view, and in contrast with multisensory convergence, multisensory integration implies that neuronal responses to the combined input of several modalities differs from the sum of responses to each modality presented individually (Avillac et al., 2007; Cléry et al., 2015b; Driver and Noesselt, 2008; Stein and Stanford, 2008). The predominant view is that the combination of information from multiple sensory modalities improves behavioral response precision and efficiency (Chen et al., 2018; Ernst and Banks, 2002; Ernst and Bülthoff, 2004; Sunkara et al., 2015). In the following, we will address multisensory convergence and integration in the context of peripersonal space and egomotion processing. These multisensory processes are most probably also involved in other contexts such as body ownership and 
social cognition (see Section 2.5). However, corresponding empirical VIP data for these scientific themes is lacking.

\subsubsection{Multisensory processing and peripersonal space}

Multisensory processing. In VIP neurons that respond to several modalities, responses to bimodal stimuli are either enhanced or depressed relative to the sum of their unimodal responses, reflecting nonlinear super- or sub-additive integrative mechanisms (Avillac et al., 2007). Bimodal responses are sometimes also evident in neurons that show unimodal responses only to one modality (Avillac et al., 2007). Although both visual-tactile and visual-auditory RFs are systematically aligned, the different modalities appear to be processed with diverging spatial reference frames. Tactile RFs appear encoded in a consistent somatotopic, head-centered reference frame (as most RFs are on the face), whereas visual RFs are intermediate between eye- and head-centered coding, with large variation from neuron to neuron (Avillac et al., 2005; Duhamel et al., 1997). Auditory RFs also vary between eye- to head-centered coding, and their coding is mostly consistent with the neuron's visual RFs coding (Schlack et al., 2005). This difference in visuo-tactile and visuo-auditory integration schemes might be related to vision and audition being "far" senses that typically encode external information, whereas somatosensation is inseparably bound to the body surface (Graziano, 2018). Moreover, note that RF mapping has relied on unisensory stimulation, and RF coding of one modality during concurrent stimulation of another has not been tested. Such experiments might shed new light on how multisensory information is integrated in space.

Convergence of all three - tactile, visual and auditory - modalities in the IPS is best investigated with fMRI. In macaque IPS, multisensory convergence is patchy: visual responses to large-field dynamic stimuli activate the entire VIP and surrounding cortex, whereas tactile face stimulation and auditory near-head stimulation elicit colocalized activations in one or two circumscribed sub-portions of VIP in each individual macaque (Guipponi et al., 2013). The presence of two patches in some animals implies that they have a duplicated face representation. In humans, group analysis of visuo-tactile-auditory convergence identifies one continuous activation in pVIP\#2 (Bremmer et al., 2001, see Figure 2), though single participant maps of this study show multiple maxima and patches in some participants; whether these represent duplicate maps cannot be determined from the published figures. In contrast, topographic maps identified with phase-encoded visual and tactile face/near-face stimulation result in duplicate maps in pVIP\#3 of some human participants (Sereno and Huang, 2006). We will pick up this point again in Section 3.

Face/head peripersonal space. The preference of VIP for stimuli near the body in all modalities has led to the proposal that VIP plays a role as part of a network of brain regions that encode peripersonal space, the region of space immediately surrounding the body (Cléry et al., 2015b; di Pellegrino and Làdavas, 2015; Graziano and Cooke, 2006). This preference was originally established based on the RF properties of single neurons, but macaque fMRI confirms the prioritization of near space for the entire region by showing that VIP activation evoked by $3 \mathrm{D}$ objects is stronger when these objects are near, rather than far, from the animal, even when retinal object size is controlled for (Cléry et al., 2018).

Yet, VIP favors stimuli near the face in all modalities. Therefore, it has been proposed that VIP specifically represents the peripersonal head space (Brozzoli et al., 2012b; Guipponi et al., 2013) by binding multisensory near-face cues either passively through multisensory convergence or actively through multisensory integration. This suggestion receives further support from a macaque fMRI study in which strong activity was evoked in VIP by a looming visual stimulus that predicted a touch to the cheek (Cléry et al., 2017), matching the psychophysical observation that such stimuli enhance the cheek's tactile-perceptual thresholds (Cléry et al., 2015a). Note, that these findings again point towards prediction as a potential overarching function of VIP, with impact prediction of stimuli looming towards the face requiring both spatial and temporal integration (Cléry et al., 2017).

In humans, potential representations of peripersonal space have often been described based on experiments that involved the hands or did not specify the reference point of the experiment's stimulation. Near stimuli result in higher IPS activations than far stimuli during an active line-bisection task with a laser pointer (Weiss et al., 2003,
2000). Similarly, anterior and posterior PPC exhibit fMRI activation when a 3D object is moved near vs. far from participants' hands (Brozzoli et al., 2011). Moreover, transcranial magnetic stimulation (TMS) applied to the right PPC impedes visual search in near but not far space, suggesting a causal involvement of PPC in near-space processing (Lane et al., 2013).

It is of note that none of these studies implicate the PPC in a specific role for representing the head and the space around it. Rather, the concept of peripersonal space is often regarded as applying to the body in general, or to the hands in particular. Early evidence for a brain region that preferentially codes peripersonal space centered on the face in humans came from human brain-lesioned patients. Some individuals show selective deficits in processing tactile stimuli on the contralesional side of the face; these deficits are modulated by visual stimuli on the ipsilesional side of the face, whereas visual stimuli distant from the face have much weaker modulatory effects (Làdavas et al., 1998). A direct investigation of the brain regions involved in face peripersonal space coding and visuo-tactile convergence in humans has led to contrasting observations. Tactile near-space topographic mapping of the face and corresponding, close-up, visual stimuli around the face reveal congruent visual and tactile maps in pVIP\#3 (Huang et al., 2017; Sereno and Huang, 2014, 2006). This area also shows activation when face-looming visual stimuli are paired with tactile stimuli on the same side of the face (Huang et al., 2018). Another fMRI study explicitly identified pVIP by contrasting a face-looming vs. static visual stimuli, found activation in pVIP\#1 (Quinlan and Culham, 2007). Thus, two different experimental protocols have led to two different localizations of pVIP, although both were based on VIP's role in face peripersonal space.

VIP does not code hand peripersonal space. Human $p$ VIP has sometimes been associated with processing of tactile stimuli on the hands, and in particular with a process referred to as tactile remapping: the transformation of tactile stimuli from an anatomical to an external-spatial reference frame (Driver and Spence, 1998). This association is based on the characteristic tactile-visual RFs of VIP neurons, combined with many, often behavioral, findings about human peripersonal spatial processing that do not highlight a particular specialization of PPC for the face, and the anatomical observation that VIP is connected with many hand tactile regions (Lewis and Van Essen, 2000b). Moreover, VIP is connected with the ventral premotor cortex, a region in which neurons also show nearspace, tactile-visual integration, for all of head, hands, arms, and torso (Graziano et al., 1997a; Graziano, 1999). On this scientific background, an influential fMRI study reported that a touchresponsive anterior PPC area combined arm posture and visual information (Lloyd et al., 2003). Specifically, when vision was available, touch to an arm crossed over to the other side of the body evoked PPC activation contralateral to the hand's anatomical position, whereas activity was ipsilateral to the anatomical position when the eyes were closed. Although macaque VIP appears to do the opposite - neurons flexibly map the visual RF to a fixed tactile RF, not vice versa -, the respective area was suggested to be the human VIP homologue. Furthermore, a macaque study on hand-centered visual coding in PPC (Obayashi et al., 2000), cited as support, neither reported to have targeted VIP, nor are the reported recording sites in the depth of the anterior or medial bank of the IPS compatible with this area; rather, the respective study likely recorded from macaque MIP, the neurons of which are involved in reaching (Colby, 1998). Subsequent studies that have attributed fMRI activation evoked by touch on the hands to pVIP have often cited the above human fMRI study as the basis for this assignment (e.g., Takahashi et al., 2013). Moreover, several human TMS studies have used the respective fMRI coordinates to disrupt either the remapping of tactile stimuli on the hands into external space (Azañón et al., 2010; Bolognini and Maravita, 2007; Renzi et al., 2013) or the visual enhancement of touch on the hand (Beck et al., 2015). Thus, TMS studies provide strong evidence that PPC is involved in processing tactile stimuli on the hands, their localization in space, and the matching between visual and tactile space. However, these functions most probably do not depend on pVIP, and it is likely that hand-related functions are mediated by neighboring regions.

VIP belongs to a cortical peripersonal space network. The peripersonal space stimuli around the face activate multiple cortical areas that can be grouped into four fronto-parietal functional subnetworks based on their main function (Cléry et al., 2015b; see 
Figure 4A, 4D): the first subnetwork contains MIP/V6A and is involved in reaching (Battaglia-Mayer et al., 2003; Fattori et al., 2017; Gamberini et al., 2009). The second subnetwork contains 7b/F5/AIP and is specialized in grasping (Menz et al., 2015; Michaels and Scherberger, 2018; Salimi et al., 1999). The third subnetwork contains F4/VIP which has been associated with selfdefense (Cooke et al., 2003; Graziano and Cooke, 2006). The fourth subnetwork is FEF/LIP, which plays a central role in oculomotor control (Ben Hamed and Duhamel, 2002; Brincat et al., 2018; Hart and Huk, 2020; Wardak et al., 2012, 2011). In addition to being coactivated with these multiple cortical areas, VIP is also connected to area 5 and $\mathrm{PFG}$, which has been proposed to contribute to the sense of body ownership (Murata and Ishida, 2007) and to the sense of agency (Fogassi et al., 2005), respectively (see section 2.4.4 for further discussion). Additionally, part of this network also belongs to the mirror neuron system (Ferrari et al., 2017; Kilner and Lemon, 2013), where mirror neurons in sensorimotor areas F5 (Gallese et al., 1996), PFG (Fogassi et al., 2005) and VIP (Ishida et al., 2010) are likely involved in recognizing actions of others (Murata and Ishida, 2008), as discussed further in Section 2.5.3.

\subsubsection{Multisensory processing and egomotion}

VIP neurons jointly encode translational and rotational visual motion components in a multiplicatively separable fashion, such that a rotation-invariant heading representation can be computed based solely on these visual cues (Sunkara et al., 2016, 2015). This strongly suggests multisensory integration between visual and vestibular information in macaques. Vestibular and visual directional responses of VIP neurons are in register, either in the same or in opposite direction (Bremmer et al., 2002b; Schlack et al., 2002). For neurons with common visual and vestibular direction, discrimination thresholds improve during bimodal stimulation. In contrast, neurons that respond to opposite directions show reduced sensitivity during bimodal stimulation (Chen et al., 2013). The presence of bimodal congruent neurons suggests integration of signals for optimal selfmotion encoding, while the presence of bimodal opposite neurons could be used for discounting retinal motion that arises from perceptually irrelevant head motion (Billington and Smith, 2015). In addition to preferred direction, velocity and acceleration are also coded differently for the two modalities. VIP's vestibular responses reflect balanced contributions of velocity and acceleration, whereas visual responses are dominated by velocity (Chen et al., 2011). Quite interestingly, neural networks that contain both congruent and incongruent neurons have recently been described as well suited for solving causal inference, i.e., deciding whether multiple signals are produced by the same or different events (Badde et al., 2021; Rideaux et al., 2021). In the context of egomotion processing, such neurons would allow self-movement and external movement to be dissociated.

Spatial codes themselves also appear to depend on the stimulation context. Earlier reports described that VIP neurons encode visual information in a continuum of eye-centered to head centered reference frames (Duhamel et al., 1997). However, the majority of neurons are eye-centered when macaques view full-field optic flow stimuli, whereas head-centered neurons are more often observed when they see single moving bar stimuli (Chen et al., 2014). These changes in the encoded reference frames involve the integration of proprioceptive eye signals with visual signals (see Section 2.1.3) and suggest that multisensory integration is task- and context-dependent. Recall that local, reversible inactivation of area VIP did not elicit any observable deficits in heading perceptual thresholds (Chen et al., 2016). This finding contrasts with those for MSTd, where inactivation did bias heading perception ( $\mathrm{Gu}$ et al., 2012) and suggests that VIP processes heading for sensorimotor transformation, and not for perceptual purposes. This is particularly intriguing because the neuronal activity of VIP is a better predictor of perceptual decisions than that of MSTd (Chen et al., 2013; Gu et al., 2008, 2007). This distinctive pattern of the two regions suggests that choice signals in VIP do not only arise from bottom-up sensory signals that determine the heading percept (in response to heading sensory cues), but also from top-down decision signals that are independent from the perceived heading. In line with this suggestion, and although some VIP neurons predicted perceptual decisions, a large part of choice signals in VIP were uncorrelated with sensory signals when the monkey had to make decisions about heading direction (Zaidel et al., 2017).

In humans, the convergence of visual and vestibular signals in VIP has been tested with fMRI by presenting visual flow that was congruent or incongruent with an instructed head turn (Schindler and Bartels, 2018). Visual-vestibular congruency activated a region that possibly matches $\mathrm{pVIP} \# 3$, though coordinates were not provided.

Other sensory cues, such as neck or trunk proprioceptive information, should also contribute to the estimation and prediction of heading direction and decisions that involve heading, but no experiments have been conducted to test this so far.

\subsection{Sensorimotor processing}

Whereas we have so far focused on sensory processing, PPC is usually conceptualized as a sensorimotor interface (Culham et al., 2006; Culham and Valyear, 2006; Gallivan and Culham, 2015; Medendorp and Heed, 2019). We turn to this aspect next.

\subsubsection{Oculomotor function}

It surprises us that the oculomotor function of VIP is mostly overlooked. Compensating for eye movements is crucial for estimating heading direction, because they alter visual motion cues on the retina (Galletti and Fattori, 2018). For instance, a moving object moves across the retina when we fixate, and the background is still. But when we visually follow the object, the object is fixed on the retina, and the background moves. VIP does not exhibit presaccadic activity, as is typically described for LIP (Morris et al., 2012). Still, an fMRI study in monkeys found activation in the ventral part of LIP and VIP during the generation of saccadic eye movements (Koyama et al., 2004). This result, however, is not confirmed by single cell recording studies, therefore fMRI activations might reflect horizontal input of LIP to VIP (Figure 4A). Such lateral connectivity might account for the remarkable stability of VIP's neuronal tuning curves in the presence of eye movements (Kaminiarz et al., 2014; Zhang et al., 2004). This stability appears to be achieved by a reduction of visual sensitivity before eye movements, and this process is specifically tuned to the planned saccade (Bremmer et al., 2009, 2017; Herrington et al., 2009; Morris et al., 2012). This finding implies that VIP has access to saccade timing and direction, either through local computations or direct input from LIP (Colby et al., 1993; Dowiasch et al., 2016; Schlack et al., 2003).

Whereas saccade-related activity appears to reach VIP indirectly, VIP is directly connected with the smooth pursuit region of the FEF (Lewis and Van Essen, 2000b; Schlag and Schlag-Rey, 2002). In line with these structural connections, the response of macaque VIP neurons is modulated by smooth pursuit and variation in current eye position (Schlack et al., 2003). On average, eye-position decoded from neuronal responses during pursuit is ahead of the true movement (Dowiasch et al., 2016), suggesting that VIP is predicting, rather than merely tracking, sensory events. In agreement with these macaque neurophysiological studies, human fMRI studies reveal that pVIP\#1 and pVIP\#2 (see Figure 3) are specifically involved in slow eye movements, including optokinetic nystagmus and smooth pursuit eye movement (Konen et al., 2005; Konen and Kastner, 2008a).

VIP belongs to an eye movement network. Although VIP is not classically listed among the areas important for eye movements, it is anatomically connected to most cortical eye movement regions for both saccades and smooth pursuit (Lewis and Van Essen, 2000b; Schlag and Schlag-Rey, 2002). The core areas of the "eye movement VIP network" (Figure 4A, 4B) are area MST for pursuit (Newsome et al., 1988) and FEF for both saccades (Bruce et al., 1985; Peel et al., 2014) and pursuit (Fukushima et al., 2010; Rosano et al., 2002). Both MST and FEF are connected to LIP, a node for both visual and saccade processing (Goldberg et al., 2006). VIP is also connected to the posterior subdivision of area 46 (Lewis and Van Essen, 2000b), an area that is thought to be in the highest level in the frontal-visual hierarchy (Schall, 2015). This oculomotor network includes additional areas, mainly through connections with FEF and LIP. The specific function of VIP in this network is unclear.

\subsubsection{Whole body motor functions}

We have already encountered several characteristics that are unique to area VIP. Yet another unique VIP feature is that its electrical stimulation can evoke complex, coordinated movements in 
macaques. Prolonged microstimulation of several hundred milliseconds induces a variety of movements that are best subsumed under head defense; these include eye closure, face grimacing, head withdrawal and movement of the hand close to the head and largely resemble natural defensive movements such as those evoked by air puffs (Cooke et al., 2003; Graziano, 2016). If stimulation continues beyond the end of the evoked movement, the animal freezes in the end posture until stimulation subsides. If stimulation is stopped prematurely, the evoked movement stops midway. Microstimulation experiments with prosimian galagos, have also identified an IPS patch that evokes movements specifically associated with defense of the face, suggesting that VIP exists in other primate species besides macaques and humans (Stepniewska et al., 2005).

Notably, the motor responses evoked by microstimulation of VIP included movements of the shoulder, arm, head, and face (Cooke et al., 2003). Thus, whereas VIP's sensory input strongly favors the face, the motor repertoire it is associated with includes interactions with the face and head, rather than just actions of these body parts. These characteristics have led to the proposal that VIP has a sensorimotor function related to coordinating complex whole body defensive behaviors to protect the head from objects near or approaching the face (Cooke et al., 2003; Graziano and Cooke, 2006). The combination of egomotion, heading direction, and defense make it plausible that VIP is involved in obstacle avoidance during locomotion (Graziano and Cooke, 2006). In other words, VIP may mediate defense and collision avoidance both when objects approach the passive animal (Cléry et al., 2017) and when the animal actively approaches objects. This idea resonates with multiple aspects we have already discussed, including differential coding of active and passive egomotion, relevance of top-down information, and the predictive character of some of VIP's computations.

In humans, there is some, albeit less direct, evidence of a motor role of $\mathrm{pVIP}$. When participants make movements with different body parts, a subregion of the topographically identified pVIP\#3 is activated by reaching movements with the arm (Huang and Sereno, 2018; Sood and Sereno, 2016). However, arm movement evokes activity in an overall much larger region, and the entire topographically mapped area cannot be matched to one specific pVIP cluster. Surprisingly, the study does not assign face movements to pVIP\#3 (Huang and Sereno, 2018). However, topographic mapping is based on assigning each MRI voxel a color based on the one single condition that most strongly activated it. Therefore, there could be sub-peak responses to other body parts that are overlooked by this method.

pVIP, and particularly pVIP\#3, has been proposed to play a role in defensive movements based on the findings in macaques and the many matching findings between the two species (Huang and Sereno, 2018), but this proposal has not yet been directly tested in humans. It has also been proposed that pVIP\#3 may be involved in coordinating hand-to-mouth eating movements in concert with neighboring AIP, which is central for grasping (Huang and Sereno, 2018). Supporting such a cooperative functional role of VIP, an experiment that directly investigated the neural responses during a natural hand-to-mouth eating movement cycle found that $\mathrm{pVIP \# 3}$ was activated when participants' reached to grasp food (Chen et al., 2019).

\subsection{Spatial coding and frames of reference}

VIP receives information from multiple sensory modalities, which are all encoded by downstream sensory regions in their own reference frame that is defined by the type and layout of the modality's sensors. For example, visual information is encoded in a retinotopic reference frame in visual cortex and many higher visionrelated areas. A given neuron will always respond to visual stimuli at a fixed location on the retina, and when the eye moves across a stationary scene, different objects will fall onto a given RF.

In contrast, auditory, vestibular, and somatosensory input is independent of the retina, and these modalities' primary cortices accordingly use coding schemes that are (at least often) incompatible with that of retinal input. For example, the location of an auditory stimulus will excite the cochlea in the same way, no matter where the eyes are directed. The spatially aligned RFs seen in VIP that match the tactile, visual, and auditory space, therefore require transformations between the respective reference frames. These transformations must take into account aspects such as eye position in the head, head posture relative to the torso and ground, and potentially the posture of other body parts. Reference frames and their transformations have been a research focus in many areas of sensorimotor neuroscience; therefore, we will briefly summarize this aspect here, although it has already surfaced in some of the previous subsections.

First, VIP encodes sensory information not only in distinct reference frames for each modality, but for some modalities, each neuron uses its own coding. These individual schemes can be described as weighted mixtures between different reference frames. For instance, a neuron's tuning function - the distribution of its firing rate in response to stimulation from different spatial locations - may be almost, but not fully, fixed to the head, and may be only slightly modulated by eye position. Thus, such a neuron might respond best to a stimulus at $45^{\circ}$ to the left of the head when the eyes look straight, but at $43^{\circ}$ when the eyes are directed $10^{\circ}$ to the right; in other words, the tuning function is only partly rather than fully shifted in the direction of eye position. A network of neurons with different reference frame weights (or tuning functions shifts) can effectively implement coordinate transformation between the two involved reference frames (Pouget et al., 2000). The net effect of these computations is that visual (Duhamel et al., 1997) and auditory information (Schlack et al., 2005) can be retrieved either in retinotopic or head-centered reference frames. Notably, even if a neuron's spatial tuning - the spatial location it best responds to - is unaffected by eye position, its firing is often modulated by an additive increase of the firing rate that is itself modulated by eye position. Thus, the neuron's reference frame is then, for example, head-centered, but an additive component, the so-termed gain field, additionally encodes eye position. Across multiple neurons, this additive coding allows information to be recovered in multiple reference frames (Snyder, 2000). Therefore, VIP appears to contain all sensory information in eye-, head- and possibly body-centered spatial codes.

Second, the weighting of different reference frames appears to change dynamically with context, suggesting that the distributed, multi-referenced coding has additional functions beyond merely computing between spatial codes and depends on the current behavioral goal. For instance, tuning functions for vestibular information are typically intermediate between body- and worldcentered when the eyes fixate while the monkey is moved - a situation in which the vestibular-ocular reflex must be actively suppressed (Chen et al., 2018). In contrast, somatosensory (Avillac et al., 2005, 2004) and vestibular tuning functions (Chen et al., 2013) are head-centered when fixation is not required.

The reference frames underlying spatial coding in PPC have also sparked much interest in human studies, but we are not aware of studies that have been specifically dedicated to exploring pVIP. Human studies often implicate larger regions of PPC (Beurze et al., 2007; Heed et al., 2011), and matching such extended activations to homologues of macaque intraparietal regions is often difficult or impossible. For the lack of being able to probe single neurons, research has relied on subtle activity changes across experimental conditions in fMRI. The experimental manipulations for such experiments resemble those in macaque single neuron studies: stimuli are presented at different locations relative to eye, head, and/or body, and it is then assessed whether changes in the fMRI signal depend on stimulus position changes in one or the other reference frame. With this approach, for example, both activation differences (Bernier and Grafton, 2010) and activation patterns across small groups of voxels (Leoné et al., 2015) in anterior PPC reflect eye-centered coding of visual reach targets, but body-centered coding of proprioceptive reach targets. These findings reiterate, for the human PPC, the task-dependence we have highlighted for macaque VIP. A third approach has been to vary the visual space during topographic mapping: maps are created for visual targets around a central fixation point, but in different runs, the fixation point is to the left, in the center, or to the right of the participant's midline. Topographic maps differ across these conditions, although visual input and saccade metrics are identical in all of them - only the general area in which stimuli and saccades take place differ-, suggesting that head or body-centered coding plays a role in a large part of PPC, including all pVIP clusters (Connolly et al., 2015). 
Other studies have explored the transformation, or remapping, of sensory stimuli from one reference frame to another. Such studies typically present stimuli on the hands, which makes it unclear whether their findings can inform us of reference frame coding in pVIP (see Section 2.2.1). Nevertheless, several PPC regions exhibit stronger responses to visual stimuli close to the hand compared to visual stimuli further away from the hand, or when the hand is not visible (Brozzoli et al., 2012a, 2011; Makin et al., 2007). These results suggest that PPC remaps visual information to match tactile location, which is consistent with the finding of macaque VIP encoding visual stimuli in a head-centered anatomical reference frame. We note that the opposite function has also been proposed as a function of PPC, with evidence of PPC being involved in remapping of tactile stimuli from an anatomical to external-spatial reference frame (Azañón et al., 2010; Lloyd et al., 2003).

Human research has also employed TMS to demonstrate changes in the spatial integration of sensory information when parietal processing is disrupted. For instance, magnetically induced phosphenes are usually more readily perceived after a touch to a hand on the same side of space - either the right or left hand, held in the space in which the phosphene is induced. In contrast, after disrupting PPC through repetitive TMS, phosphene detection is facilitated only by the touched hand's body side (e.g., left hand for left phosphene), no matter whether that hand is placed in the left or right space (Bolognini and Maravita, 2007). Thus, PPC matches the external (or visual) spatial location of a touch with input from other modalities, suggesting that human PPC contains eye-centered reference frames - a conclusion further corroborated by several other TMS studies (Azañón et al., 2010; Renzi et al., 2013; also see Section 2.2.1). Yet, as discussed in Section 2.2.1, these studies most likely targeted other, neighboring regions and not VIP in the human brain.

In sum, experimental work on reference frame coding and transformation in the human brain is abundant but has not targeted pVIP. Therefore, many results from human studies demonstrate close similarities between macaque and human parietal function, but the regional/anatomical matching between the two species has often remained at a coarse level.

\subsection{Higher cognitive functions}

As is the case for the PPC in general (see, e.g., Chafee and Crowe, 2013; Chivukula et al., 2019; Freedman and Assad, 2011; Freedman and Ibos, 2018; Stoet and Snyder, 2004) VIP is also involved in higher cognitive functions. So far, VIP has been found to play a role in the processing of numerosity, auditory sequences, and working memory. We suspect that VIP is additionally associated with other cognitive functions, especially in relation to social cognition as well as self and body awareness. We will turn to these currently unexplored topics in Section 3.

\subsubsection{Numerosity and enumeration}

Electrophysiological recordings in macaques demonstrate that VIP neurons respond to specific numerosities when macaques view sets of dots (Nieder et al., 2006; Nieder and Miller, 2004; RamirezCardenas et al., 2016; Vallentin et al., 2012; Viswanathan and Nieder, 2015, 2013) and can even encode empty sets as a null quantity (Ramirez-Cardenas et al., 2016). VIP has a higher proportion of numerosity-selective neurons than neighboring regions, (Nieder and Miller, 2004) and its numerosity responses occur spontaneously, even when monkeys are not trained in the respective tasks (Viswanathan and Nieder, 2013). In fact, training does not increase the decodability of numerosity from VIP (Viswanathan and Nieder, 2015). VIP neurons also appear to carry numerical information when macaques must switch between two number-related tasks (Vallentin et al., 2012), hinting at a role for VIP in higher-level executive functions. At first sight, numerosity may seem unrelated to all other functions that characterize VIP; however, we will suggest in Section 3 that numerosity coding can be conceptualized in a spatial prediction error framework, which may naturally link numerosity with VIP's remaining functions.

In humans, the IPS is active when participants have to detect target numbers among other numbers, as compared to letters and colors, for both visual and auditory stimulus presentation (Eger et al., 2003). The IPS also exhibits habituation effects for visual dot displays that scale with the difference in dot number of the test stimulus from the habituation stimulus (Piazza et al., 2004). Thus, parietal number representations are accessed automatically in the absence of any explicit numerical comparison, reminiscent of the independence of macaque VIP numerosity responses from task and training. Although the reported activations are compatible with our pVIP complex, a specific association with $\mathrm{pVIP} \# 1, \# 2$, or \#3 is not possible based on the available reports. Yet, when pVIP is explicitly defined with the trimodal method of pVIP\#2, the identified area represents numerosity independent of spatial location, evident in multi-voxel pattern decoding of dot number for arrays displayed at different screen locations (Eger et al., 2015).

Besides VIP, numerosity coding occurs in lateral prefrontal cortex (IPFC) (Figure 4A, 4E). Three physiological parameters suggest that VIP is hierarchically below IPFC. First, number-related responses arise earlier in VIP than IPFC (Nieder and Miller, 2004; Viswanathan and Nieder, 2013). Second, co-varying sensory features modulate neurons more in VIP than IPFC (Nieder and Miller, 2004), suggesting a higher level of abstraction in IPFC than VIP. Third, individual VIP neurons respond either to visual or auditory numbers, but not both, whereas IPFC neurons respond to both modalities (Nieder, 2012), again suggesting stronger abstraction in IPFC.

VIP neurons not only keep track of concurrently visible items; they also integrate item numbers in visual and auditory stimulus sequences, such as the total number of sequentially presented dots (Nieder et al., 2006). Furthermore, tonal sequences that violate an expected sequence pattern activate a frontoparietal network that includes VIP in macaques (Uhrig et al., 2014) if violations concern tone numbers, but not when they concern pitch (Wang et al., 2015). Thus, VIP is involved in visual and auditory enumeration, and sensitivity to sequences implies that VIP performs temporal prediction (see Section 3).

\subsubsection{Working memory}

VIP's ability to count sequential items implies that the region integrates information over time. This, in turn, requires encoding and upholding information in working memory (Baddeley, 2003). Macaque VIP neurons maintain numerosity responses during a delay period (Jacob and Nieder, 2014; Ramirez-Cardenas and Nieder, 2019) and are less affected by distractor stimuli during the delay than dlPFC neurons (Jacob and Nieder, 2014). Whether these working memory capabilities are specific to the context of numerosity, or whether they generalize to other cognitive contexts - for instance, holding a goal heading direction or a peripersonal location in memory - is presently unknown. Such abilities are important when animals must withhold an action until a moving target is in the right place, and when they navigate in cluttered environments where potentially harmful objects are temporarily hidden from view and change location due to their own, or the animal's, movement. In fact, PMv, which is directly connected with VIP (Figure 4A, 4E) and whose neurons also exhibit peripersonal-spatial characteristics, maintains the location of visual objects after the room has turned dark (Graziano et al., 1997b). However, to our knowledge it is not clear whether PMv receives this continued information from VIP, or whether it independently upholds this information after VIP ceases forwarding visual information. 


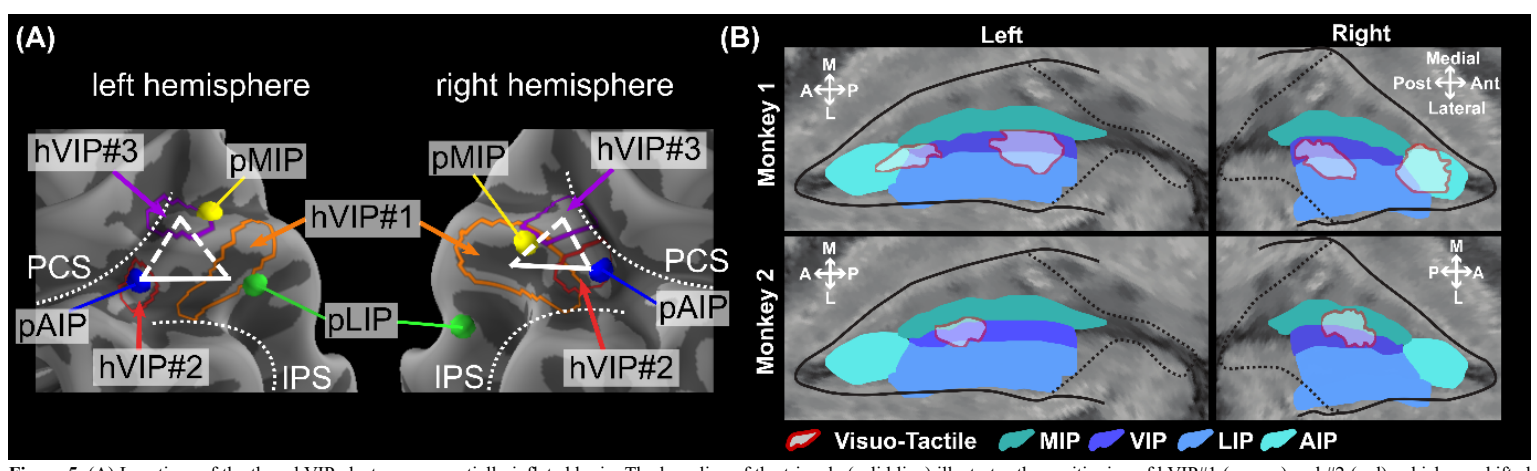

Figure 5. (A) Locations of the three hVIP clusters on a partially inflated brain. The base line of the triangle (solid line) illustrates the positioning of hVIP\#1 (orange) and \#2 (red), which are shifted medially and rotated approximately $45^{\circ}$ relative to the fundus of the IPS. The dashed lines of the triangle illustrate the proposed expansion of hVIP\#3 (purple) into the lateral bank of the PCS. The dotted lines mark the IPS and PCS. The locations of LIP (green), AIP (blue) and MIP (yellow) illustrate the layout of VIP within the larger organization of PCC. (B) Regions expressing functional visuo-tactile convergence zones in VIP, referred to as vtVIPa and vtVIPp (top). The other monkey has only one convergence zone, referred to as vtVIP (bottom).

2.5.3 Body ownership and bodily self-consciousness Body ownership is the awareness that our body and its different parts belong to us. It is thought to be one of the main correlates of subjective experience, the process by which one binds external and internal experience to one's own self (Blanke and Metzinger, 2009; Gallagher, 2000). Ownership over hands, face, and body relies on the integration of multiple bodily signals, including tactile, proprioceptive, visual and auditory signals (Blanke et al., 2015; Ehrsson et al., 2004; Makin et al., 2008; Serino et al., 2013; Tsakiris, 2008). These findings have led to proposals of a direct link between the neural mechanisms underlying body ownership and peripersonal space (Blanke, 2012; Blanke et al., 2015; Cléry \& Ben Hamed, 2018; Makin et al., 2008). A recent coordinate-based meta-analysis of human functional neuroimaging studies identified the cortical regions that likely contribute to these two processes (Grivaz et al., 2017). A conjunction analysis revealed two parietal regions that are activated across both peripersonal space and body ownership studies. At closer inspection, these activations can be associated with the area between $p$ VIP\#2 and \#3 and a cortical region anterior to it (see Figure $3 \mathrm{~B}$, green spheres). While some aspects of body representation have been addressed in macaques, for instance using the rubber hand illusion (Fang et al., 2019; Graziano et al., 2000), we are not aware that body ownership has been addressed in macaque VIP.

\subsubsection{Social cognition}

Recent research has demonstrated that peripersonal space has a strong social component in its dynamics (Cléry et al., 2015b; Cléry and Ben Hamed, 2020, 2018). For example, the presence of an observer and the nature of her/his interaction with oneself reshapes the peripersonal space of human participants (Heed et al., 2010; Pellencin et al., 2018; Teneggi et al., 2013). Such findings have led to the hypothesis that the brain encodes a shared, inter-personal peripersonal space. Indeed, events that take place near another person's face improve the salience of stimuli occurring in one's own peripersonal space (Maister et al., 2015). Likewise, the detection of tactile stimuli on human participants' own hands are faster when a visual stimulus approaches the hand of another person (Teramoto, 2018).

Macaque VIP contains some neurons that respond to tactile stimulation of both their own face and the face of the experimenter (Ishida et al., 2010). The same study reported that some VIP neurons respond to visual stimulation near the monkey's face, i.e., in its peripersonal space, as well as near to the experimenter's face.

We will expand on the possible relevance of these, and potentially other, unexplored social aspects for understanding VIP in Section 3.

\section{Evidence-based definition of human hVIP:} synthesis and suggestions for future research

We have now assembled an overview over past research on macaque VIP and the attempts to define its possible human homologue. It is evident that the region is involved in a plethora of sensorimotor and higher cognitive functions in the macaque, but a function-to-region mapping is difficult for the human brain. In this section, we will draw together these diverse findings. We will first argue that the three human PPC regions that are commonly identified as putative human
VIP homologues together form the human VIP homologue that we now term "hVIP complex". Its three composing clusters will accordingly be called hVIP\#1, hVIP\#2 and hVIP3. We will then discuss evidence that suggests that this complex is not one large area but, instead, has specialized from a single multifunctional area into three separate areas in the human brain during primate brain evolution. Along the way, we will present some speculative ideas and suggest some routes for future research.

\subsection{The human hVIP complex}

Several findings have suggested that the overall regional organization of human PPC is largely preserved compared to the PPC of macaques. Homologues have been suggested for macaque IPS regions AIP, LIP, and posterior intraparietal area (PIP) based on functional similarities and anatomical considerations (Orban, 2016). Similarly, homologues have been suggested for macaque regions V6, V6A, PEc and PE (also termed area 5), which are all located in a posterior-to-anterior strip medial of IPS in both macaques and humans (Gamberini et al., 2020; Pitzalis et al., 2019). These widespread similarities between the two species make it probable that homologies also exist for PPC regions that have not yet been unambiguously matched. At the same time, many parts of the human cortex have significantly expanded relative to those of the macaque (Chaplin et al., 2013; Hill et al., 2010; Orban et al., 2004; see also discussion in Gamerbini et al., 2019), including PPC and the IPS in particular (Orban et al., 2004). For instance, VIP's neighbor LIP has probably diversified into three distinct regions in human PPC (termed IPS1-3; Kastner et al., 2017), evident in multiple retinotopographic maps along the IPS (Konen and Kastner, 2008; Schluppeck et al., 2005; Sereno et al., 2001; Silver et al., 2005). So what about human VIP?

3.1.1 A large human hVIP complex as the functional homologue of monkey VIP

Research on macaque VIP has mostly focused on low-level functions, whereas human VIP research has often aimed at higherlevel cognitive functions. Nonetheless, our review has identified three functional clusters in human anterior PPC that each share some functional properties with macaque VIP. Two of these clusters, hVIP\#1 and hVIP\#2, are located in the middle third of the IPS fundus (see Fig. 5). The third cluster, hVIP\#3, lies medially of the IPS, on the lateral bank of the PCS (see Fig. 5). Together, these three clusters encompass the functional properties of macaque VIP, but importantly, none of them appears to match all functions of macaque VIP on its own. Therefore, we propose that the three hVIP clusters together form the human hVIP complex.

Macaque VIP is cytoarchitectonically well-defined (see Fig. 1), and the regions that surround it are all involved in key sensorimotor functions. Medially, VIP borders MIP and area 5, both predominantly involved in reach planning and arm-eye coordination. Laterally, it borders LIP, an area predominantly involved in eye movement coordination. Anteriorly, it borders AIP, thought to be predominantly involved in grasping. Finally, posteriorly it borders the posterior intraparietal area (PIP), which is predominantly specialized in $3 \mathrm{D}$ object coding. 


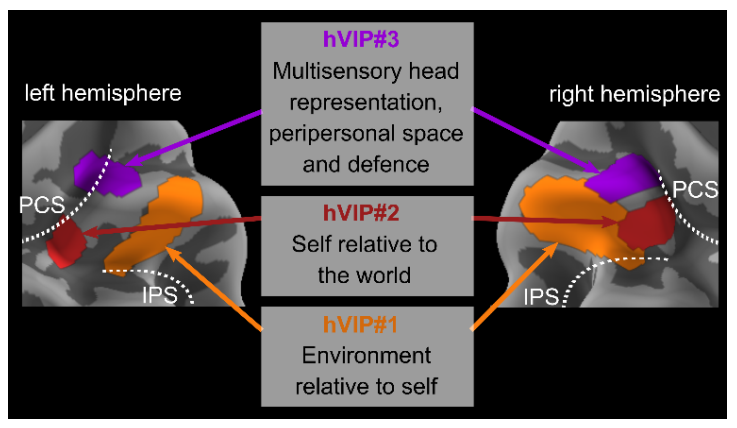

Figure 6. Proposed functions of the three components of the human VIP complex.

It is far beyond our scope here to critically review all of these PPC regions in detail. We have thus based their estimated locations on reference studies that have relied on low-level sensorimotor tasks (see Fig. 5). A comparison of these presumed locations together with those of our hVIP\#1-3 regions suggests that the vicinity of the human hVIP complex and its surrounding putative homologues of MIP, LIP and AIP preserve the macaque PPC's functionally specified regional organization. Strikingly, the hVIP cluster appears to partly colocalize with the peak activations reported for LIP, MIP, and AIP, respectively (Fig. 2, especially the left hemisphere). This overlap may, in part, be due to variability in the reported locations of the different regions: studies usually report a single voxel as the center of the respective regions of interest, and the precise location of such a core voxel varies with individual anatomy, employed statistical procedures, etc. Alternatively, the regional overlap may reflect uncertainty about the underlying functions thought to be central for localizing the respective areas in the human brain. In other words, previous studies may have misnamed areas because - just like in the case of VIP - consensus about the experimental manipulation(s) adequate to identify the core function of a given region is lacking. Both possibilities - variability across studies and uncertainty about relevant functions - call for meta-analysis of human functional data that integrates knowledge beyond individual areas. Moreover, it may be advantageous to purposely combine multiple experimental manipulations thought to be central to different areas in one experiment to test for potential functional overlap.

Although the regional organization of human IPS appears largely preserved compared to macaques, the layout of the hVIP complex relative to the IPS fundus appears shifted medially, as well as rotated by roughly $45^{\circ}$ in humans, so that it covers both the fundus and a substantial part of the medial bank of the IPS, as well as part of the lateral bank of the PCS (see Fig. 5). Notably, the proposed shift and rotation relative to the IPS is compatible with the observation that cortical expansion in humans relative to macaques has been more prominent around the temporo-parietal junction (TPJ) and the inferior PPC than in medial PPC (Chaplin et al., 2013; Hill et al., 2010): the expanding inferior PPC would have pushed lateral regions to more medial locations. From a functional perspective, it has been pointed out that the weaker expansion of human medial areas such as area 5 predicts larger functional overlap across the two species in superior than in inferior parietal cortex (Gamberini et al., 2020). In contrast, the function of the TPJ is considered highly specific to human cognition, given its massive expansion during evolution [although a homologue of human TPJ has been proposed in the macaque temporal sulcus, (Mars et al., 2013)]. We suggest that the cortex in the region of the hVIP complex shows an intermediate level of expansion relative to the low-expansion area 5 and high-expansion inferior parietal cortex. According to this argument, the human hVIP complex should share important common functions with macaque VIP but should also exhibit human-specific functions. As of yet, evidence in this respect is sparse, but we will pick up this idea again at the end of this review.

If the three pVIP clusters reflect an expanded VIP, then what does the cortex between these three clusters do? In our review, we have relied on reported coordinates, that is, single voxels that summarize a reported region's location, usually the peak activation or the center of gravity of a patch determined with fMRI. Accordingly, Fig. 3 summarizes point estimates over multiple human VIP studies, and the actual reported activations extended around these points, possibly encompassing the cortex inbetween the three hVIP clusters and, potentially, exhibiting overlap. Given the distribution of functions across the three clusters, each reviewed study identifies part of the computations implemented by the illustrated patch, but the individual experimental conditions may not reflect the clusters' predominant function(s). Moreover, we re-iterate that the outlines shown in Fig. $2 \mathrm{~A}$ merely reflect illustrative, visually-estimated summaries of the previously reported average locations and are not based on anatomical or functional delineations - in fact, those entries in Figure $2 \mathrm{~A}$ that seem to be outliers may actually indicate functional overlap rather than error variance. This open point leads to our next question, namely whether the proposed human hVIP complex corresponds to a unique functional area, or whether it has diversified into three distinct functional subregions.

3.1.2 Functional specialization within the human hVIP complex

Fig. 6 illustrates a tentative specification of the overarching functional specialization of each hVIP cluster. This attempt of functional separation could guide systematic exploration in future experiments.

3.1.3 Functional specialization within macaque VIP

The idea of specialized subregions within a larger VIP complex would receive significant support if a comparable functional specialization also existed in macaques. Firm conclusions in this regard are difficult: VIP-centered functional imaging studies in macaques are rare, and single cell studies often do not reconstruct their recording locations precisely relative to the IPS. Nonetheless, two independent lines of research lend support to the idea of a functional specialization within macaque VIP similar to that we have suggested above for human hVIP.

The first is the cytoarchitectonic separation of macaque VIP into medial and lateral regions, VIPm and VIPl (Lewis and Van Essen, 2000a). To date, no functional specialization has been associated with these two subsections. The two strips are so thin that standard fMRI protocols with a voxel size of $1-1.5 \mathrm{~mm}^{3}$ are too coarse to reliably capture functional differences. Single cell recordings might provide insights if neuronal responses are precisely mapped - an endeavor that has not yet been undertaken. Even though the functional significance of this division has not been explored, it is conceivable that it has served as a basis for the medio-lateral expansion of VIP in the human brain. Second, macaque fMRI supports the existence of a functional gradient in the antero-posterior direction (see Fig. 5B) - that is, orthogonal to the cytoarchitectonically identified medio-lateral split (Guipponi et al., 2015, 2013; Wardak et al., 2016). Along the anterior-posterior axis, only a small, anterior part of VIP showed both visuo-tactile properties and face preference, just like the human anterior-lateral hVIP\#2. In contrast, posterior VIP was activated by visual large-field dynamic stimuli, reminiscent of the human posterior hVIP\#1. Thus, a previously overlooked functional specialization may exist within macaque VIP that precedes the more obvious specialization of the human hVIP complex.

What's more, recall that visuo-tactile convergence patterns in VIP varied between individual macaques, with some individuals exhibiting one, and others two, multisensory convergence zones in VIP (Guipponi et al., 2013; see Section 2.2.1, regions vtVIPa and vtVIPp). In topographic mapping some, but not all, human participants also had duplicate face maps in hVIP\#3, and one of them extended towards the IPS fundus (Huang et al., 2017). One could map the two-zone VIP monkey pattern with the human hVIP complex layout by assigning anterior macaque vtVIPa to human $\mathrm{hVIP} \# 2$ and posterior macaque vtVIPp to human hVIP\#3. In fact, functional considerations support this assignment: vtVIPp showed stronger selectivity for near-face stimuli (Cléry et al., 2018) and predicted impact to the face better (Cléry et al., 2017) than vtVIPa, consistent with visuotactile face maps located in hVIP\#3. Moreover, this scheme could be complemented by the visually responsive posterior portion of macaque VIP beyond vtVIPp as a match for the visually-focused human hVIP\#1. Notably, this scheme makes the (currently untested) prediction that macaque vtVIPa should respond to neck proprioception, and vtVIPp should possibly exhibit face mirroring responses. These ideas are speculative but outline a roadmap for further exploring the functional specialization, areal organization, and homology of macaque and human VIP. 


\section{2 hVIP: One or multiple functional areas}

So far, we have highlighted the functional organization of VIP under a perspective of regional expansion, with a focus on assessing homology between macaque and human. An alternative (nonexclusive) possibility to regional preservation is that the splitting of one VIP in macaques into three regions in humans reflects the emergence of new, distinct functional areas that have developed under distinct species-specific evolutionary pressure to provide qualitatively distinct abilities. The classification of a cortical region as distinct can be based on at least four different aspects (Orban, 2016; Orban et al., 2004): (i) cyto- and myeloarchitectonics, (ii) anatomical connectivity with other areas, (iii) topographic organization with respect to the surrounding areas, and (iv) functional properties. We discuss macaque VIP and the three hVIP areas under these four criteria.

\subsubsection{Cytoarchitectonic delineation of VIP}

Human cytoarchitectonic studies of PPC are still sparse (Amunts et al., 2020; Richter et al., 2019; Scheperjans et al., 2008; see Section 1 and Fig. 2B). Merging the functionally identified hVIP clusters with a cytoarchitectonically-based parcellation of PPC is not straightforward. Our hVIP clusters are currently tentative and defined only visually. On the other hand, both cytoarchitectonic and fMRI studies rely on a small number of individual brains or participants and inevitably exhibit idiosyncratic variability. Moreover, whereas the hVIP clusters are relatively symmetric across hemispheres, human PPC cytoarchitectonic maps are not (Richter et al., 2019; Scheperjans et al., 2008). Nonetheless, when we align the functionally-based hVIP cluster outlines with the available cytoarchitectonic PPC parcellation, each hVIP cluster falls into distinct cytoarchitectonic region(s), indicating that the clusters' neuronal substrate probably differs, possibly pointing toward diversification into cortical areas rather than functional specialization within a common area. However, functional hVIP\#1 overlaps with multiple cytoarchitectonic areas (7A, hIP3, hIP1, 7P, hIP6; see Section 1), which is difficult to reconcile with a function-to-anatomymatching approach. Similar reservations exist for hVIP\#2 and \#3. In sum, alignment of available functional and anatomical maps does not render a consistent parcellation, and the reliability of the cytoarchitectonic-to-functional correspondence is unclear. Nonetheless, mapping is consistent with each hVIP cluster comprising a distinct anatomical substrate.

\subsubsection{Anatomical and functional connectivity of VIP}

Both anatomical and functional connectivity patterns are informative about a region's functional role (Gamberini et al., 2020; Mars et al., 2018; Vijayakumar et al., 2018) and cross-species functional homology (Orban, 2016). Our review of macaque anatomical VIP connectivity has revealed detailed knowledge of multiple functionally distinct networks, but equivalent data is non-existent for human hVIP. One study has parcellated the human brain based on connectivity as assessed with diffusion-weighted tensor imaging (DTI). This approach divided PPC into 5 large regions, one of which overlapped with hVIP\#3 (Mars et al., 2011) but extended well beyond our outline. Therefore, it may match our entire hVIP complex, or it may correspond to only one or two clusters, which would suggest a (partly) independent parcellation scheme.

Contrary to the sparse data on human anatomical connectivity, there is rich functional human data that indirectly identifies larger networks that co-activate with human hVIP through task-based fMRI studies (see Section 2). Ideally, new research should address the anatomical connectivity of VIP in humans and its functional connectivity in the macaque. The latter effort is already ongoing (for examples, see Cléry et al., 2018, 2017; Guipponi et al., 2015) and will likely increase thanks to data sharing initiatives such as the international Prime-DRE consortium on non-human primate neuroimaging (Milham et al., 2020, 2018) and use of common localizers (Russ et al., 2021). Given the difficulty and scope of assessing multi-subject cytoarchitectonics in humans (Amunts et al., 2020), functional connectivity is probably more promising than anatomical connectivity for investigating VIP homology.

One approach to characterize functional connectivity is to systematically observe which areas co-activate with hVIP for specific (sub-)functions. Yet, few human studies have specifically focused on hVIP in this regard. Instead, hVIP has usually been identified as one region among many involved in a given functional context. A region-specific focus will provide stronger grounds for understanding the functional specificity of each hVIP cluster. At the same time, it will direct macaque research towards precisely investigating possible regional specialization within monkey VIP. This goal will critically depend on systematic, strictly comparative approaches that employ task-based fMRI with identical experimental conditions across macaque and human.

Besides a task-based approach, connectivity could be characterized through high resolution resting state fMRI functional connectivity. Previous work has parcellated cortex through clustering of functional resting state data in both humans (Glasser et al., 2016) and macaques (Vijayakumar et al., 2018). The human study integrated multiple anatomical and functional criteria, combining architectural, functional, connectivity and topographical data. The human VIP that resulted from this integrative approach, however, does not agree with any of the clusters we have described. In macaques, resting state parcellation revealed two small clusters that overlaid VIP (Vijayakumar et al., 2018). Notably, the aim of clustering approaches is to reduce complexity by uncovering similarities and ignoring small differences of neighboring pieces of cortex (e.g., MRI voxels). We propose that, alternatively, differences, rather than commonalities, in connectivity profiles could be exploited to characterize regional specialization. Public availability of resting state and DTI datasets make these kinds of MR-based approaches promising for characterizing macaque-human homology.

\subsubsection{Functional topographic mapping and functional specificity}

Given VIP's multisensory and sensorimotor properties, functional maps and topographical organization could be explored from multiples perspectives: visual, somatosensory, vestibular, auditory, proprioceptive, oculomotor, hand-motor, and likely more. To date, the potential topographical organization of area VIP, or of PPC including VIP, has been investigated for only some of these properties. Interestingly, this functional mapping seems to support a dissociation of the human hVIP clusters into independent functional maps.

When assessed with phase-encoded mapping (Sereno et al., 2001), both executing and planning saccades to neighboring locations on the retina (and, thus, neighboring locations in space while the eyes fixate) reveal orderly maps in multiple areas of PPC, suggesting that neighboring neurons respond to neighboring spatial locations during eye movements. PPC exhibits at least 5 retinotopic maps along the posterior-to-anterior axis of PPC (Konen and Kastner, 2008a). Visual comparison of these mapping results with our Fig. 2 suggests that functional cluster hVIP\#2 overlaps with the so-defined IPS5, while functional cluster hVIP\#1 appears to overlap with IPS4, supporting the idea that the hVIP clusters are functionally distinct cortical areas. Notably, phase-encoded mapping identified the retinotopic organization of IPS $4 / \mathrm{hVIP \# 1} \mathrm{both} \mathrm{when} \mathrm{static} \mathrm{and} \mathrm{motion} \mathrm{visual}$ stimuli were used, whereas retinotopy in IPS5/hVIP\#2 was evident only with motion stimuli (Konen and Kastner, 2008; Sereno et al., 2001; Sereno and Huang, 2006). Phase-encoded mapping has also been used to explore cortical organization with respect to motor functions (Huang and Sereno, 2018). Saccade-related and smooth pursuit maps seem to exist in IPS4/hVIP\#1 and IPS5/hVIP\#2, but not in hVIP\#3 (Konen et al., 2005).

Classical phase-encoded, retinotopic visual and saccade mapping did not identify a topographical map at the location of our functional hVIP\#3 cluster (Konen and Kastner, 2008). In an attempt to match human and macaque PPC regions, this region was subsequently defined as the human parietal grasp region (hPGR; Kastner et al., 2017), that is, the homologue of macaque area AIP. However, phaseencoded visual retinotopic mapping did identify a topographically organized representation of the contralateral visual field in hVIP\#3 when stimuli were presented near the face (Huang et al., 2017). Thus, each of the three hVIP clusters appears topographically organized, though for different stimulus characteristics. Also note, that matching topographic maps to otherwise defined regions is not always straight-forward, and the very close vicinity of AIP and hVIP (see Fig. 2A) is evident here when near-space topographic mapping is compared to grasping responses.

Somatosensory responses have also been explored with phaseencoded mapping in humans (Chen et al., 2017; Huang et al., 2018, 
2012, 2012; Huang and Sereno, 2018). Tactile stimuli were applied by airpuffs delivered to multiple body parts, or to multiple locations on the face. Face stimulation revealed a contralateral map which precisely matched the topographic maps obtained through visual near-face stimulation, including inter-individual variability in size and map duplication (Huang et al., 2017). Somatosensory maps were evident only in hVIP\#3, but not in hVIP\#1 and hVIP\#2, thus singling out hVIP\#3 as a tactile-visual convergence zone. When applying phase-encoding mapping across the whole body (Huang et al., 2012), pVIP\#3 was found to be part of a larger whole-body homunculus, where finger responses were found directly anterior to hVIP\#2, and shoulder, leg and foot responses were found medial to hVIP\#3. Thus pVIP\#3 is part of a topographically mapped whole body strip that lies anterior to the rest of the hVIP complex. fMRI-based somatosensory phase-encoded mapping has not been applied in macaques. However, tactile and visual RFs of single neurons in macaque area 5 have been mapped in anesthetized macaques (Seelke et al., 2012). In this area, which includes the medial bank of the IPS that neighbors VIP, the dominant body part is the hand, in strong contrast to the face-dominant VIP. The human topographic maps predict that neurons in macaque VIP should also be organized in an orderly manner with respect to tactile and visual response properties. Yet, single-cell recordings have not so far revealed any identifiable organization of neuronal tuning (Duhamel et al., 1998).

Overall, phase-encoded functional mapping of PPC identifies distinct, topographically organized maps for several sensory modalities and motor functions for hVIP\#1, \#2, and \#3, consistent with functional dissociations between them. This method would principally be well-suited for use in macaques. Furthermore, the combination of fMRI- and electrophysiology-based methods could enhance our understanding of PPC topographic organization across multiple spatial scales.

Functional, connectional, cytoarchitectonic, and macroorganizational mapping all provide evidence for a separation of the three hVIP clusters, even if not all evidence is unequivocal. Viewed in sum, the three human hVIP clusters are probably each distinct areas that have evolved as an expansion of the single area VIP in macaques. The areas surrounding VIP/hVIP are similar in humans and macaques, suggesting that VIP and the three hVIPs, respectively, also have similar functionality across the two species. Yet, the three hVIPs exhibit functional specialization, a feature that may already be detectable along the main axis of macaque VIP.

\subsection{An evolutionary perspective on VIP}

The macaque monkey has been the prime non-human primate model in neuroscience for decades. However, a larger view across the evolutionary landscape can often aid a more complete understanding of how, and possibly why, the human brain has developed its current organization (Krubitzer, 2009; Krubitzer and Disbrow, 2008; Krubitzer and Prescott, 2018). Primate evolution is thought to have branched 3 times between our common primate ancestor and today's human race: the earliest split-off are the Prosimians, such as the prosimian galago. The second split-off are the New World monkeys, such as the common marmoset. The third split separates the Old World monkeys, including the Old World macaque that is neuroscience's most important primate species, from the Great Apes and Hominids. Some of VIP's functions appear critical for all primate species, for example the processing of visual motion and the prioritized processing of peripersonal space in connection with defensive behaviors and self-feeding. Have these functions been pooled in a specialized area in primates from an early evolutionary time point, and might a comparative view of such a region's development give us an indication of the functions it provides in the human brain today?

It is unclear whether a cortical region with core VIP-like properties exists in the lissencephalic prosimian species such as marmose monkeys. In New World marmoset monkeys, histology-based reference atlases do not identify a homologue of macaque area VIP, and the homologue of area LIP is directly medial to the homologue of MIP (Paxinos et al., 2011). An fMRI study presented marmoset monkeys with looming visual stimuli, which would reliably activate VIP in macaques. Marmosets showed activations in the LIP homologue, but not in MIP and V6A (Cléry et al., 2017). A direct contrast between looming and receding visual stimuli revealed only a small cortical region at the border between marmoset LIP and MIP (i.e., at the expected location of VIP), and only in the right hemisphere.

Taken together, this evidence seems to suggest that VIP may not exist in New World marmosets. However, electrical microstimulation in anterior PPC evoked defensive movements in prosimian galagos - a lineage that split off earlier from the remaining species than New World monkeys. Microstimulation evoked defensive movements with the face for lateral stimulation sites, and with the forelimb for more medial stimulation sites (Kaas and Stepniewska, 2016), proving the existence of (some) VIP-like functionality in this species. If there is indeed a VIP homologue in this species, then we see VIPlike regional organization in the oldest and newest evolutionary lineages, and would accordingly expect that VIP exists also in New World marmosets. Attempting to trigger defensive movements via microstimulation might be a better functional marker of core VIP across species than the response to looming visual stimuli.

Overall, these findings point towards a preserved core cortical area across species that perceives imminent physical threat and protects the body, although a direct test of this highly specialized VIP function has not been performed in humans (Huang and Sereno, 2018). VIP's overrepresentation of the head also appears consistent from this perspective: arguably, the head is the most important part of any primate body. It contains the brain and almost all sensory organs - especially those relevant to perceiving distant objects (vision, audition) and the body in the world (vision, vestibular sensation). It also contains the mouth, which is not only the point of food intake, but is also central for primate vocal communication. The evolutionary pressure to achieve effective protection of this body part must be high, and may be higher than for other body parts. In fact, if this line of thought bears truth, a core VIP should be identifiable in other mammals.

Although there is currently no evidence for a dedicated representation of peripersonal space around body parts other than the head in macaque PPC, peripersonal visual-tactile RFs at least on the arms and torso do exist in premotor cortex, which is directly connected with VIP (Graziano et al., 1997a, 1994). Furthermore, human topographic mapping of tactile responses has suggested that hVIP\#3 may be part of a much larger strip of anterior PPC that responds to touch and vision (Sereno and Huang, 2014). Thus, future research may uncover peripersonal responses for the rest of the body in PPC as well. Of course, VIP does a lot more than coding peripersonal head space and defensive actions, and thus the focus on the head may also be driven by other factors beyond merely the higher value of the head relative to the remaining body.

\subsection{Conclusions and outlook: what does VIP really do?}

As we draw our exploration of macaque and human VIP to a close, does a conclusive and integrated picture emerge from the many different aspects and viewpoints we have addressed?

Throughout the paper, we have mentioned conclusions and speculations related to specific points. During this discourse, we have cleared up two apparently widespread misconceptions about the human VIP homologue. First and foremost, hVIP is not an area that, in its entirety, can be defined by just a subset of the functions that have been identified in the macaque: all methods that have been frequently used to identify the human VIP homologue reliably identify one of three clusters that cover the complete functionality of the macaque area only in combination. Second, hVIP is not an area that remaps skin input into 3D space for the entire body. VIP's capabilities in representing and transforming between multiple spatial reference frames support this idea, albeit specifically related to the head and face.

A review that viewed PPC from a perspective of providing state estimation of the body and world to enable motor action has suggested that the PPC exhibits a gradient-like organization between linking the world to the body and, vice versa, projecting the body into the world (Medendorp and Heed, 2019). These two poles enable different sensorimotor and cognitive functionality that focus either on abstract action planning towards objects in the environment or on concrete action implementation and body control. In this framework, hVIP appears to do both (see Fig. 6). Furthermore, a key property of macaque VIP neurons is the flexible binding of visual space onto fixed, tactile RFs for the face and head. Thus, the region appears to 
link visual space to the skin (rather than projecting touch into space). However, the proposed dual role of VIP in both protecting the body and navigating it through the environment would require both knowledge about the world and body action. These two-poled requirements may be at the core of the individuation of the more anterior, tactile-visual hVIP\#3 from the posterior, visual and motionfocused hVIP\#1.

The expansion into multiple areas and, accordingly, a more dedicated specialization towards the body and world poles, may have laid the basis for at least some of the presumed higher functions of the human hVIP complex, perhaps similar to the suspected emergence of finegrained tool use abilities with that of a specialized anterior-lateral PPC area in humans (Kastner et al., 2017). We will raise several nonexclusive possibilities here.

First, enhanced facial-peripersonal spatial processing may have been important for social and cooperative behavior. This hypothesis has been previously proposed (Graziano and Cooke, 2006), with the suggestion that the coding of peripersonal space may be instrumental in defining the distance between individuals in different social contexts and signaling trust by presenting vulnerable body parts, such as the belly area in dogs. This general suggestion is corroborated by VIP's facial mirror properties which are likely critical to empathic interpretation of other's emotional experiences and a crucial aspect of human communication skills. Recall further, that VIP also responds to social vocalization, which adds yet further evidence that it probably plays a role in social behavior.

Second, the central role of the head in navigation and action may have promoted the development of perceiving a self. The activation of the area between hVIP\#2 and \#3 during tasks that involve body awareness (Grivaz et al., 2017) indicates that this seemingly complex function ultimately relies on basic sensorimotor processing. This consideration is also supported by the central role of multisensory integration for body ownership (Blanke et al., 2015), another function in which hVIP\#2 and \#3 are involved. Bodily selfconsciousness and awareness, in particular of the head and face, have been demonstrated in humans and apes (Kaneko and Tomonaga, 2012, 2011; Kopp et al., 2021), but are yet unexplored in other primate species. Moreover, when human participants point towards themselves (which other species never do), they aim with a bias to the head away from the body's center of gravity (Alsmith and Longo, 2014; van der Veer et al., 2018), possibly reflecting an implicit bias that emerges from the prominent spatial representation of the head.

Third, a focus on the head as the hub of the self may have promoted other social behaviors expressed by humans. Humanity has put particular emphasis on the head and face from very early on in cultural development, in the form of haircuts, face painting and make-up, hats etc., either for aesthetic, communicative, or protective purposes. In addition to these behaviors that purposely enhance awareness of the head and face, humans have developed a large range of head-directed hand gestures that support implicit communication; for example, we hide the mouth with the hand when we lie and touch our hair when we are insecure, to name just two examples. The neurobiological origin of these head-face gestures is unclear and can already be observed in some apes. For example, chimpanzees are anecdotally described to wipe their nose with the back of their hand at moments of peak uncertainty (Catherine Crockford, personal communication). Gorillas are also reported to place their hands in front of their face to conceal their face and thus hide their mood, for example, to hide a playface (Tanner and Byrne, 1993). We have discussed the strong connections of VIP with hand motor regions that is involved with protection of the head with the hands. This same connectivity could also support oral communication and the use of the hands in communicative gestures, connecting with the social and communicative aspects we have discussed above.

Lastly, in humans the dramatic expansion of a substantial part of PPC has been associated with tool use (Kastner et al., 2017). In monkeys, morphological and anatomical changes in the medial bank of the IPS, covered by areas MIP and AIP, have been associated with tool use (Hihara et al., 2006; Obayashi et al., 2001, 2000; Quallo et al., 2009). This suggests that some neurons in macaque PPC usually responsible for the hands can also respond to tools after training (Iriki et al., 1996). VIP has also been associated with tool-use ability (Graziano, 2018), and many human behavioral, neuropsychological, and neuroimaging studies have emphasized a relationship between tooluse, peripersonal space and body representation (Maravita and Iriki,
2004; Maravita and Romano, 2018; Martel et al., 2019; Serino, 2019). Yet, it is unclear whether the respective changes in structure and function include VIP. If so, there are two non-mutually exclusive possibilities for how tool-use may have modulated VIP differentiation and expansion in humans. First, humans are generally capable of learning to control tools with the mouth, as is impressively demonstrated by people who were born without hands. But even when the hands are normally developed, humans use straws to drink with and master musical instruments through complex mouth-toobject interaction. Thus, VIP may take over such mouth-related tooluse. Second, humans often use the mouth in support of hand actions, for instance, to crush, grip, or tear, or to aid tool use in activities such as sewing. Here, VIP would be relevant due to its putative role in hand-to-face coordinated behavior. However, these ideas are currently purely speculative.

Amongst all the functional properties associated with VIP, numerosity and enumeration stand out and seem difficult to relate to the low-level sensory properties of this area. We think that it is possible to bind the seemingly diverse functions of VIP, such as impact prediction, sequence violation, and numerosity, as a signature of specific neuronal computations related to prediction. Indeed, impact prediction and sequence violation can be considered as two faces of the same coin: the former involves the confirmation of predictive coding and the latter consists of the representation of a prediction error. In addition, one involves temporal prediction (sequence violation) while the other involves spatial and temporal prediction, both for impact prediction to the body and heading estimation. Moreover, assessing the number of objects in the immediate environment may be important for determining an adequate response, such as whether to make a defensive arm movement vs. a ducking movement vs. turning and fleeing. Based on these considerations, numerosity can be viewed in the context of spatial prediction, and enumeration in the context of temporal prediction. One way to approach this idea could be to model whether neurons tuned to spatial prediction are able to perform temporal prediction, and whether such neurons contain information about numerosity.

Clearly, many of this section's suggestions are far from hard evidence. Not all of them may turn out true. Nonetheless, we hope that by placing VIP in contexts in which it has not previously been implicated will inspire future research in humans as well as across species.

To summarize, we have assembled multiple lines of evidence that all suggest that macaque area VIP has developed into three areas in the human brain, which we have termed the hVIP complex. Each of these areas performs a subset of macaque VIP's functions, though precursors of a functional division may already be present in that species. Although VIP and hVIP are involved in many sensorimotor and cognitive functions, we have outlined some possible overarching themes. Determining which, if any, of these proposals are correct awaits dedicated investigation.

\section{Acknowledgements}

This work was supported by the German Research Foundation (DFG) and the French National Research Agency (ANR) through grants He 6368/4-1 to TH and ANR-18-CE92-0048 to SBH in the DFG/ANR programme for German-French Projects in the Natural, Life, and Engineering Sciences and LABEX CORTEX funding ANR-11-LABX-0042 from the Université de Lyon from the programme Investissements d'Avenir (ANR-11-IDEX-0007) operated by the ANR.

Thank you, Janina Klautke, for raising the VIP discussion back in the day.

\section{Data statement}

Code and data used to generate Figures 2, 3, 5 and 6 is available at: https://osf.io/nce $7 \mathrm{x} / \mathrm{hVIP}$ complex outlines are provided in both surface-space and volume-space. 


\section{References}

Aedo-Jury, F., Cottereau, B.R., Celebrini, S., Séverac Cauquil, A., 2020. AnteroPosterior vs. Lateral Vestibular Input Processing in Human Visual Cortex. Front Integr Neurosci 14, 43. https://doi.org/10.3389/fnint.2020.00043

Albright, T.D., 1984. Direction and orientation selectivity of neurons in visual area MT of the macaque. J Neurophysiol 52, 1106-1130.
https://doi.org/10.1152/jn.1984.52.6.1106

Alsmith, A.J.T., Longo, M.R., 2014. Where exactly am I? Self-location judgements distribute between head and torso. Consciousness and Cognition 24, 70-74. https://doi.org/10.1016/j.concog.2013.12.005

Amunts, K., Mohlberg, H., Bludau, S., Zilles, K., 2020. Julich-Brain: A 3D probabilistic atlas of the human brain's cytoarchitecture. Science 369, 988-992. https://doi.org/10.1126/science.abb4588

Avila, E., Lakshminarasimhan, K.J., DeAngelis, G.C., Angelaki, D.E., 2019. Visual and Vestibular Selectivity for Self-Motion in Macaque Posterior Parietal Area 7a. Cereb Cortex 29, 3932-3947. https://doi.org/10.1093/cercor/bhy272

Avillac, M., Ben Hamed, S., Duhamel, J.-R., 2007. Multisensory Integration in the Ventral Intraparietal Area of the Macaque Monkey. J. Neurosci. 27, 1922-1932. https://doi.org/10.1523/JNEUROSCI.2646-06.2007

Avillac, M., Denève, S., Olivier, E., Pouget, A., Duhamel, J.-R., 2005. Reference frames for representing visual and tactile locations in parietal cortex. Nat Neurosci 8, 941-949. https://doi.org/10.1038/nn1480

Avillac, M., Olivier, E., Denève, S., Ben Hamed, S., Duhamel, J.-R., 2004 Multisensory integration in multiple reference frames in the posterior parietal cortex. Cogn Process 5, 159-166. https://doi.org/10.1007/s10339-004-0021-3

Azañón, E., Longo, M.R., Soto-Faraco, S., Haggard, P., 2010. The Posterio Parietal Cortex Remaps Touch into External Space. Curr. Biol. 20, 1304-1309. https://doi.org/10.1016/j.cub.2010.05.063

Badde, S., Hong, F., Landy, M.S., 2021. Causal inference and the evolution of opposite neurons. PNAS 118. https://doi.org/10.1073/pnas.2112686118

Baddeley, A., 2003. Working memory: looking back and looking forward. Nat Rey Neurosci 4, 829-839. https://doi.org/10.1038/nrn1201

Bartels, A., Zeki, S., Logothetis, N.K., 2008. Natural Vision Reveals Regional Specialization to Local Motion and to Contrast-Invariant, Global Flow in the Human Brain Cerebral https://doi.org/10.1093/cercor/bhm107

Batista, A.P., Buneo, C.A., Snyder, L.H., Andersen, R.A., 1999. Reach plans in

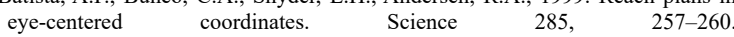
https://doi.org/10.1126/science.285.5425.257

Battaglia-Mayer, A., Caminiti, R., Lacquaniti, F., Zago, M., 2003. Multiple Levels of Representation of Reaching in the Parieto-frontal Network. Cereb Cortex 13, 1009-1022. https://doi.org/10.1093/cercor/13.10.1009

Beck, B., Bertini, C., Haggard, P., Làdavas, E., 2015. Dissociable routes fo personal and interpersonal visual enhancement of touch. Cortex 73, 289-297. https://doi.org/10.1016/j.cortex.2015.09.008

Ben Hamed, S., 1999. Caracterisation fonctionnelle de la representation visuelle de l'aire intraparietale laterale (lip): specificites et modulation comportementales (PhD Dissertation). http://www.theses.fr. Paris 6.

Ben Hamed, S., Duhamel, J.-R., 2002. Ocular fixation and visual activity in the monkey lateral intraparietal area. Exp Brain Res 142, 512-528. https://doi.org/10.1007/s00221-001-0954-z

Ben Hamed, S., Page, W., Duffy, C., Pouget, A., 2003. MSTd neuronal basis functions for the population encoding of heading direction. J Neurophysiol 90 549-558. https://doi.org/10.1152/jn.00639.2002

Bernier, P.-M., Grafton, S.T., 2010. Human posterior parietal cortex flexibly determines feference frames for reaching based on sensory context. Neuron 68 , 776-788. https://doi.org/10.1016/j.neuron.2010.11.002

Beurze, S.M., de Lange, F.P., Toni, I., Medendorp, W.P., 2007. Integration of Target and Effector Information in the Human Brain During Reach Planning. J Neurophysiol 97, 188-199. https://doi.org/10.1152/jn.00456.2006

Billington, J., Smith, A.T., 2015. Neural Mechanisms for Discounting Head-RollInduced Retinal Motion. J. Neurosci. 35, 4851-4856 https://doi.org/10.1523/JNEUROSCI.3640-14.2015

Binkofski, F., Buccino, G., Stephan, K.M., Rizzolatti, G., Seitz, R.J., Freund, H.J., 1999. A parieto-premotor network for object manipulation: evidence from neuroimaging. Exp Brain Res 128, 210-213. https://oi.org/10.1007/s002210050838

Bizley, J.K., Cohen, Y.E., 2013. The what, where and how of auditory-object perception. Nat Rev Neurosci 14, 693-707. https://doi.org/10.1038/nrn3565

Blanke, O., 2012. Multisensory brain mechanisms of bodily self-consciousness. Nat Rev Neurosci 13, 556-571. https://doi.org/10.1038/nrn3292

Blanke, O., Metzinger, T., 2009. Full-body illusions and minimal phenomenal selfhood. Trends Cogn Sci 13, 7-13. https://doi.org/10.1016/j.tics.2008.10.003 Blanke, O., Slater, M., Serino, A., 2015. Behavioral, Neural, and Computationa Principles of Bodily Self-Consciousness. Neuron 88, 145-166. https://doi.org/10.1016/j.neuron.2015.09.029

Bolognini, N., Maravita, A., 2007. Proprioceptive Alignment of Visual and Somatosensory Maps in the Posterior Parietal Cortex. Curr. Biol. 17, 1890-1895. https://doi.org/10.1016/j.cub.2007.09.057

Boussaoud, D., Ungerleider, L.G., Desimone, R., 1990. Pathways for motion analysis: Cortical connections of the medial superior temporal and fundus of the superior temporal visual areas in the macaque. Journal of Comparative Neurology 296, 462-495. https://doi.org/10.1002/cne.902960311

Bremmer, F., Churan, J., Lappe, M., 2017. Heading representations in primates are compressed by saccades. Nat Commun 8,920 . https://doi.org/10.1038/s41467017-01021-5

Bremmer, F., Duhamel, J.-R., Ben Hamed, S., Graf, W., 2002a. Heading encoding in the macaque ventral intraparietal area (VIP). European Journal of Neuroscience 16, 1554-1568. https://doi.org/10.1046/j.1460-9568.2002.02207.x

Bremmer, F., Klam, F., Duhamel, J.-R., Ben Hamed, S., Graf, W., 2002b. Visualvestibular interactive responses in the macaque ventral intraparietal area (VIP).
European Journal of Neuroscience 16, 1569-1586. https://doi.org/10.1046/j.1460-9568.2002.02206.x

Bremmer, F., Kubischik, M., Hoffmann, K.-P., Krekelberg, B., 2009. Neural dynamics of saccadic suppression. J. Neurosci. 29, 12374-12383. https://doi.org/10.1523/JNEUROSCI.2908-09.2009

Bremmer, F., Schlack, A., Kaminiarz, A., Hoffmann, K.P., 2013. Encoding of movement in near extrapersonal space in primate area VIP. Front. Behav. Neurosci. 7. https://doi.org/10.3389/fnbeh.2013.00008

Bremmer, F., Schlack, A., Shah, N.J., Zafiris, O., Kubischik, M., Hoffmann, K.-P., Zilles, K., Fink, G.R., 2001. Polymodal Motion Processing in Posterior Parietal and Premotor Cortex: A Human fMRI Study Strongly Implies Equivalencies between Humans and Monkeys. Neuron 29, 287-296. https://doi.org/10.1016/s0896-6273(01)00198-2

Brewer, A.A., Barton, B., 2016. Maps of the Auditory Cortex. Annu. Rev. Neurosci. 39, 385-407. https://doi.org/10.1146/annurev-neuro-070815-014045 Brincat, S.L., Siegel, M., von Nicolai, C., Miller, E.K., 2018. Gradual progression from sensory to task-related processing in cerebral cortex. Proc Natl Acad Sci USA 115, E7202-E7211. https://doi.org/10.1073/pnas.1717075115

Britten, K.H., 2008. Mechanisms of self-motion perception. Annu. Rev. Neurosci. 31, 389-410. https://doi.org/10.1146/annurev.neuro.29.051605.112953

Brozzoli, C., Gentile, G., Ehrsson, H.H., 2012a. That's Near My Hand! Parietal and Premotor Coding of Hand-Centered Space Contributes to Localization and Self-Attribution of the Hand. J. Neurosci. 32, 14573-14582. https://doi.org/10.1523/JNEUROSCI.2660-12.2012

Brozzoli, C., Gentile, G., Petkova, V.I., Ehrsson, H.H., 2011. fMRI Adaptation Reveals a Cortical Mechanism for the Coding of Space Near the Hand. J. Neurosci. 31, 9023-9031. https://doi.org/10.1523/JNEUROSCI.1172-11.2011

Brozzoli, C., Makin, T.R., Cardinali, L., Holmes, N.P., Farnè, A., 2012b. Peripersonal Space: A Multisensory Interface for Body-Object Interactions, in: Murray, M.M., Wallace, M.T. (Eds.), The Neural Bases of Multisensory Processes, Frontiers in Neuroscience. CRC Press/Taylor \& Francis, Boca Raton (FL).

Bruce, C.J., Goldberg, M.E., Bushnell, M.C., Stanton, G.B., 1985. Primate frontal eye fields. II. Physiological and anatomical correlates of electrically evoked eye $\begin{array}{llll}\text { movements. } & \text { J } & \text { Neurophysiol } & \text { 714-734. }\end{array}$ https://doi.org/10.1152/jn.1985.54.3.714

Cardin, V. Smith, A. T, 2010. Sensitivity of Human Visual and Vestibular Cortical Regions to Egomotion-Compatible Visual Stimulation. Cereb Cortex 20, 19641973. https://doi.org/10.1093/cercor/bhp268

Cardini, F., Costantini, M., Galati, G., Romani, G.L., Làdavas, E., Serino, A., 2011. Viewing one's own face being touched modulates tactile perception: an fMRI study. J Cogn Neurosci 23, 503-513. https://doi.org/10.1162/jocn.2010.21484

Cavina-Pratesi, C., Goodale, M.A., Culham, J.C., 2007. FMRI reveals a dissociation between grasping and perceiving the size of real 3D objects. PLoS One 2, e424. https://doi.org/10.1371/journal.pone.0000424

Chafee, M.V., Crowe, D.A., 2013. Thinking in spatial terms: decoupling spatial representation from sensorimotor control in monkey posterior parietal areas $7 \mathrm{a}$ and LIP. Front Integr Neurosci 6, 112. https://doi.org/10.3389/fnint.2012.00112 Chaplin, T.A., Yu, H.-H., Soares, J.G.M., Gattass, R., Rosa, M.G.P., 2013. A Conserved Pattern of Differential Expansion of Cortical Areas in Simian

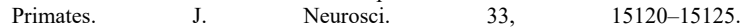
https://doi.org/10.1523/JNEUROSCI.2909-13.2013

Chen, A., DeAngelis, G.C., Angelaki, D.E., 2011. Representation of Vestibular and Visual Cues to Self-Motion in Ventral Intraparietal Cortex. J. Neurosci. 31, 12036-12052. https://doi.org/10.1523/JNEUROSCI.0395-11.2011

Chen, A., Gu, Y., Liu, S., DeAngelis, G.C., Angelaki, D.E., 2016. Evidence for a Causal Contribution of Macaque Vestibular, But Not Intraparietal, Cortex to Heading Perception. J. Neurosci. 36, 3789-3798. https://doi.org/10.1523/JNEUROSCI.2485-15.2016

Chen, C.-F., Kreutz-Delgado, K., Sereno, M.I., Huang, R.-S., 2019. Unraveling the spatiotemporal brain dynamics during a simulated reach-to-eat task. NeuroImage 185, 58-71. https://doi.org/10.1016/j.neuroimage.2018.10.028

Chen, C.-F., Kreutz-Delgado, K., Sereno, M.I., Huang, R.-S., 2017. Validation of periodic fMRI signals in response to wearable tactile stimulation. NeuroImage 150, 99-111. https://doi.org/10.1016/j.neuroimage.2017.02.024

Chen, X., DeAngelis, G.C., Angelaki, D.E., 2018. Flexible egocentric and allocentric representations of heading signals in parietal cortex. Proc. Natl. Acad. Sci. U.S.A. 115, E3305-E3312. https://doi.org/10.1073/pnas.1715625115 Chen, X., DeAngelis, G.C., Angelaki, D.E., 2014. Eye-centered visual receptive fields in the ventral intraparietal area. J. Neurophysiol. 112, 353-361. https://doi.org/10.1152/jn.00057.2014

Chen, X., DeAngelis, G.C., Angelaki, D.E., 2013. Diverse Spatial Reference Frames of Vestibular Signals in Parietal Cortex. Neuron 80, 1310-1321. https://doi.org/10.1016/j.neuron.2013.09.006

Chivukula, S., Jafari, M., Aflalo, T., Yong, N.A., Pouratian, N., 2019. Cognition in Sensorimotor Control: Interfacing With the Posterior Parietal Cortex. Front. Neurosci. 13, 140. https://doi.org/10.3389/fnins.2019.00140

Churan, J., Kaminiarz, A., Schwenk, J.C.B., Bremmer, F., 2021. Action-dependent processing of self-motion in parietal cortex of macaque monkeys. J Neurophysiol 125, 2432-2443. https://doi.org/10.1152/jn.00049.2021

Cléry, J., Ben Hamed, S., 2020. Functional networks for peripersonal space coding and prediction of impact to the body, in: de Vignemont, F., Serino, A., Wong, H.Y., Farnè, A. (Eds.), The World at Our Fingertips: A Multidisciplinary Exploration of Peripersonal Space. Oxford University Press, Oxford, England.

Cléry, J., Ben Hamed, S., 2018. Frontier of Self and Impact Prediction. Front. Psychol. 9, 1073. https://doi.org/10.3389/fpsyg.2018.01073

Cléry, J., Guipponi, O., Odouard, S., Pinède, S., Wardak, C., Ben Hamed, S., 2017. The Prediction of Impact of a Looming Stimulus onto the Body Is Subserved by Multisensory Integration Mechanisms. J. Neurosci. 37, 10656-10670. https://doi.org/10.1523/JNEUROSCI.0610-17.2017 
Cléry, J., Guipponi, O., Odouard, S., Wardak, C., Ben Hamed, S., 2018 Cortical networks for encoding near and far space in the non-human primate. NeuroImage 176, 164-178. https://doi.org/10.1016/j.neuroimage.2018.04.036

Cléry, J., Guipponi, O., Odouard, S., Wardak, C., Ben Hamed, S., 2015a. Impact prediction by looming visual stimuli enhances tactile detection. J. Neurosci. 35 , 4179-4189. https://doi.org/10.1523/JNEUROSCI.3031-14.2015

Cléry, J., Guipponi, O., Wardak, C., Ben Hamed, S., 2015b. Neuronal bases of peripersonal and extrapersonal spaces, their plasticity and their dynamics: Knowns and unknowns. Neuropsychologia 70, 313-326. https://doi.org/10.1016/j.neuropsychologia.2014.10.022

Colby, C.L., 1998. Action-Oriented Spatial Reference Frames in Cortex. Neuron 20, 15-24. https://doi.org/10.1016/S0896-6273(00)80429-8

Colby, C.L., Duhamel, J.R., Goldberg, M.E., 1993. Ventral intraparietal area of the macaque: anatomic location and visual response properties. J. Neurophysiol. 69 , 902-914. https://doi.org/10.1152/jn. 1993.69.3.902

Connolly, J.D., Vuong, Q.C., Thiele, A., 2015. Gaze-dependent topography in human posterior parietal cortex. Cereb Cortex 25, 1519-1526. https://doi.org/10.1093/cercor/bht344

Cook, E.P., Maunsell, J.H.R., 2002a. Attentional modulation of behaviora performance and neuronal responses in middle temporal and ventral intraparieta areas of macaque monkey. J. Neurosci. 22, 1994-2004. https://doi.org/10.1523/JNEUROSCI.22-05-01994.2002

Cook, E.P., Maunsell, J.H.R., 2002b. Dynamics of neuronal responses in macaque MT and VIP during motion detection. Nat Neurosci 5, 985-994. https://doi.org/10.1038/nn924

Cooke, D.F., Taylor, C.S.R., Moore, T., Graziano, M.S.A., 2003. Complex movements evoked by microstimulation of the ventral intraparietal area. PNAS 100, 6163-6168. https://doi.org/10.1073/pnas. 1031751100

Cottereau, B.R., Smith, A.T., Rima, S., Fize, D., Héjia-Brichard, Y., Renaud, L. Lejards, C., Vayssière, N., Trotter, Y, Durand, J.-B., 2017. Processing of Egomotion-Consistent Optic Flow in the Rhesus Macaque Cortex. Cereb Cortex 27, 330-343. https://doi.org/10.1093/cercor/bhw412

Culham, J.C., Cavina-Pratesi, C., Singhal, A., 2006. The role of parietal cortex in visuomotor control: What have we learned from neuroimaging? Neuropsychologia 44, https://doi.org/10.1016/j.neuropsychologia.2005.11.003

Culham, J.C., Danckert, S.L., DeSouza, J.F.X., Gati, J.S., Menon, R.S., Goodale, M.A., 2003. Visually guided grasping produces fMRI activation in dorsal but not ventral stream brain areas. Exp Brain Res 153, 180-189. https://doi.org/10.1007/s00221-003-1591-5

Culham, J.C., Valyear, K.F., 2006. Human parietal cortex in action. Curren Opinion in Neurobiology 16, 205-212. https://doi.org/10.1016/j.conb.2006.03.005

Delhaye, B.P., Long, K.H., Bensmaia, S.J., 2018. Neural Basis of Touch and Proprioception in Primate Cortex. Compr Physiol 8, 1575-1602. https://doi.org/10.1002/cphy.c170033

Di Marco, S., Fattori, P., Galati, G., Galletti, C., Lappe, M., Maltempo, T., Serra C., Sulpizio, V., Pitzalis, S., 2021. Preference for locomotion-compatible curve paths and forward direction of self-motion in somatomotor and visual areas. Cortex 137, 74-92. https://doi.org/10.1016/j.cortex.2020.12.021

di Pellegrino, G., Làdavas, E., 2015. Peripersonal space in the brain Neuropsychologia $66,126-133$ https://doi.org/10.1016/j.neuropsychologia.2014.11.011

Dowiasch, S., Blohm, G., Bremmer, F., 2016. Neural correlate of spatial (mis)localization during smooth eye movements. European Journal of Neuroscience 44, 1846-1855. https://doi.org/10.1111/ejn.13276

Driver, J., Noesselt, T., 2008. Multisensory Interplay Reveals Crossmodal Influences on 'Sensory-Specific' Brain Regions, Neural Responses, and Judgments. Neuron 57, 11-23. https://doi.org/10.1016/j.neuron.2007.12.013

Driver, J., Spence, C., 1998. Cross-modal links in spatial attention. Phil. Trans. R. Soc. Lond. B 353, 1319-1331. https://doi.org/10.1098/rstb.1998.0286 Duffy, C.J., Wurtz, R.H., 1991. Sensitivity of MST Neurons to Optic Flow Stimuli. I. A Continuum of Response Selectivity to Large-Field Stimuli. J Neurophysiol 65, 1329-1345. https://doi.org/10.1152/jn.1991.65.6.1329

Duhamel, J.-R., Bremmer, F., Ben Hamed, S., Graf, W., 1997. Spatial invariance of visual receptive fields in parietal cortex neurons. Nature $389,845-848$. https://doi.org/10.1038/39865

Duhamel, J.-R., Colby, C.L., Goldberg, M.E., 1998. Ventral intraparietal area of the macaque: congruent visual and somatic response properties. J. Neurophysiol. 79, 126-136. https://doi.org/10.1152/jn.1998.79.1.126

Eger, E., Pinel, P., Dehaene, S., Kleinschmidt, A., 2015. Spatially Invariant Coding of Numerical Information in Functionally Defined Subregions of Human Parietal Cortex. Cereb Cortex 25, 1319-1329. https://doi.org/10.1093/cercor/bht323

Eger, E., Sterzer, P., Russ, M.O., Giraud, A.-L., Kleinschmidt, A., 2003. A Supramodal Number Representation in Human Intraparietal Cortex. Neuron 37 719-726. https://doi.org/10.1016/S0896-6273(03)00036-9

Ehrsson, H.H., Spence, C., Passingham, R.E., 2004. That's My Hand! Activity in Premotor Cortex Reflects Feeling of Ownership of a Limb. Science 305, 875877. https://doi.org/10.1126/science.1097011

Ernst, M.O., Banks, M.S., 2002. Humans integrate visual and haptic information in a statistically optimal fashion. Nature 415, 429-433. https://doi.org/10.1038/415429a

Ernst, M.O., Bülthoff, H.H., 2004. Merging the senses into a robust percept. Trend in Cognitive Sciences 8, 162-169. https://doi.org/10.1016/j.tics.2004.02.002

Fang, W., Li, J., Qi, G., Li, S., Sigman, M., Wang, L., 2019. Statistical inference of body representation in the macaque brain. PNAS 116, 20151-20157. https://doi.org/10.1073/pnas.1902334116

Fasold, O., Heinau, J., Trenner, M.U., Villringer, A., Wenzel, R., 2008. Proprioceptive head posture-related processing in human polysensory cortical areas. NeuroImage $\quad 40, \quad 1232-1242$. https://doi.org/10.1016/j.neuroimage.2007.12.060
Fattori, P., Breveglieri, R., Bosco, A., Gamberini, M., Galletti, C., 2017. Vision for Prehension in the Medial Parietal Cortex Cereb. Cortex 27, 1149-1163. https://doi.org/10.1093/cercor/bhv302

Ferrari, P.F., Gerbella, M., Coudé, G., Rozzi, S., 2017. Two different mirror neuron networks: The sensorimotor (hand) and limbic (face) pathways. Neuroscience 358, 300-315. https://doi.org/10.1016/j.neuroscience.2017.06.052

Ferri, S., Rizzolatti, G., Orban, G.A., 2015. The organization of the posterior parietal cortex devoted to upper limb actions: An fMRI study. Hum. Brain Mapp. 36, 3845-3866. https://doi.org $/ 10.1002 / \mathrm{hbm} .22882$

Field, D.T., Biagi, N., Inman, L.A., 2020. The role of the ventral intraparietal area (VIP/pVIP) in the perception of object-motion and self-motion. NeuroImage 213, 116679. https://doi.org/10.1016/j.neuroimage.2020.116679

Fogassi, L., Ferrari, P.F., Gesierich, B., Rozzi, S., Chersi, F., Rizzolatti, G., 2005. Parietal Lobe: From Action Organization to Intention Understanding. Science 308, 662-667. https://doi.org/10.1126/science.1106138

Freedman, D.J., Assad, J.A., 2011. A proposed common neural mechanism for categorization and perceptual decisions. Nat Neurosci 14, 143-146. https://doi.org/10.1038/nn.2740

Freedman, D.J., Ibos, G., 2018. An Integrative Framework for Sensory, Motor, and Cognitive Functions of the Posterior Parietal Cortex. Neuron 97, 1219-1234. https://doi.org/10.1016/j.neuron.2018.01.044

Frey, S.H., Vinton, D., Norlund, R., Grafton, S.T., 2005. Cortical topography of human anterior intraparietal cortex active during visually guided grasping. Cognitive Brain Research https://doi.org/10.1016/j.cogbrainres.2004.11.010

Froesel, M., Gacoin, M., Clavagnier, S., Hauser, M., Goudard, Q., Ben Hamed, S., 2021. Neural correlates of audio-visual integration of socially meaningful information in macaque monkeys. bioRxiv 2021.05.02.442333. https://doi.org/10.1101/2021.05.02.442333

Fukushima, K., Akao, T., Saito, H., Kurkin, S.A., Fukushima, J., Peterson, B.W., 2010. Representation of Neck Velocity and Neck-Vestibular Interactions in Pursuit Neurons in the Simian Frontal Eye Fields. Cereb Cortex 20, 1195-1207. https://doi.org/10.1093/cercor/bhp180

Furlan, M., Wann, J.P., Smith, A.T., 2014. A Representation of Changing Heading Direction in Human Cortical Areas pVIP and CSv. Cereb Cortex 24, 2848-2858. https://doi.org/10.1093/cercor/bht132

Gabel, S., Misslisch, H., Gielen, C., Duysens, J., 2002. Responses of neurons in area VIP to self-induced and external visual motion. Exp Brain Res 147, 520528. https://doi.org/10.1007/s00221-002-1268-5

Gallagher, S., 2000. Philosophical conceptions of the self: implications for cognitive science. Trends in Cognitive Sciences 4, 14-21. https://doi.org/10.1016/S1364-6613(99)01417-5

Gallese, V., Fadiga, L., Fogassi, L., Rizzolatti, G., 1996. Action recognition in the premotor cortex. Brain 119,593-609. https://doi.org/10.1093/brain/119.2.593

Galletti, C., Battaglini, P.P., Fattori, P., 1991. Functional Properties of Neurons in the Anterior Bank of the Parieto-occipital Sulcus of the Macaque Monkey. Eur J Neurosci 3, 452-461. https://doi.org/10.1111/j.1460-9568.1991.tb00832.x

Galletti, C., Fattori, P., 2018. The dorsal visual stream revisited: Stable circuits or dynamic pathways? Cortex 98, 203-217.

https://doi.org/10.1016/j.cortex.2017.01.009

Galletti, C., Gamberini, M., Kutz, D.F., Fattori, P., Luppino, G., Matelli, M., 2001. The cortical connections of area V6: an occipito-parietal network processing visual information. European Journal of Neuroscience 13, 1572-1588. https://doi.org/10.1046/j.0953-816x.2001.01538.x

Gallivan, J.P., Culham, J.C., 2015. Neural coding within human brain areas involved in actions. Current Opinion in Neurobiology 33, 141-149. https://doi.org/10.1016/j.conb.2015.03.012

Gamberini, M., Passarelli, L., Fattori, P., Galletti, C., 2020. Structural connectivity and functional properties of the macaque superior parietal lobule. Brain Struct Funct 225, 1349-1367. https://doi.org/10.1007/s00429-019-01976-9

Gamberini, M., Passarelli, L., Fattori, P., Zucchelli, M., Bakola, S., Luppino, G., Galletti, C., 2009. Cortical connections of the visuomotor parietooccipital area V6Ad of the macaque monkey. J Comp Neurol 513, 622-642. https://doi.org/10.1002/cne.21980

Gattass, R., Sousa, A.P., Gross, C.G., 1988. Visuotopic organization and extent of V3 and V4 of the macaque. J. Neurosci. 8, 1831-1845. https://doi.org/10.1523/JNEUROSCI.08-06-01831.1988

Glasser, M.F., Coalson, T.S., Robinson, E.C., Hacker, C.D., Harwell, J., Yacoub, E., Ugurbil, K., Andersson, J., Beckmann, C.F., Jenkinson, M., Smith, S.M., Van Essen, D.C., 2016. A multi-modal parcellation of human cerebral cortex. Nature 536, 171-178. https://doi.org/10.1038/nature18933

Goldberg, M.E., Bisley, J.W., Powell, K.D., Gottlieb, J., 2006. Chapter 10 Saccades, salience and attention: the role of the lateral intraparietal area in visual behavior, in: Progress in Brain Research. Elsevier, pp. 157-175. https://doi.org/10.1016/S0079-6123(06)55010-1

Goodwin, G.M., McCloskey, D.I., Matthews, P.B.C., 1972. Proprioceptive Illusions Induced by Muscle Vibration: Contribution by Muscle Spindles to Perception? Science 175, 1382-1384. https://doi.org/10.1126/science.175.4028.1382

Graziano, M., 2018. The Spaces Between Us: A Story of Neuroscience, Evolution, and Human Nature. Oxford University Press, Oxford, New York.

Graziano, M.S.A., 2016. Ethological Action Maps: A Paradigm Shift for the Motor Cortex. Trends Cogn Sci. 20, 121-132. https://doi.org/10.1016/j.tics.2015.10.008 Graziano, M.S.A., 1999. Where is my arm? The relative role of vision and proprioception in the neuronal representation of limb position. Proceedings of the National Academy of Sciences 96, 10418-10421. https://doi.org/10.1073/pnas.96.18.10418

Graziano, M.S.A., Cooke, D.F., 2006. Parieto-frontal interactions, personal space, and defensive behavior. Neuropsychologia 44, 845-859. https://doi.org/10.1016/j.neuropsychologia.2005.09.009 
Graziano, M.S.A., Cooke, D.F., Taylor, C.S.R., 2000. Coding the Location of the Arm by Sight. Science 290, 1782-1786. https://doi.org/10.1126/science.290.5497.1782

Graziano, M.S.A., Hu, X.T., Gross, C.G., 1997a. Visuospatial Properties of Ventral Premotor Cortex. J Neurophysiol 77, 2268-2292. https://doi.org/10.1152/jn.1997.77.5.2268

Graziano, M.S.A., Hu, X.T. Gross, C.G., 1997b. Coding the Locations of Objects in the Dark. Science 277, 239-241. https://doi.org/10.1126/science.277.5323.239 Graziano, M.S.A., Yap, G.S., Gross, C.G., 1994. Coding of visual space by

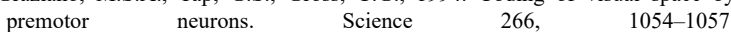
https://doi.org/10.1126/science.7973661

Grefkes, C., Ritzl, A., Zilles, K., Fink, G.R., 2004. Human medial intraparietal cortex subserves visuomotor coordinate transformation. NeuroImage 23, 1494 1506. https://doi.org/10.1016/j.neuroimage.2004.08.031

Grivaz, P., Blanke, O., Serino, A., 2017. Common and distinct brain regions processing multisensory bodily signals for peripersonal space and body ownership. bodily signa $147, \quad 602-618$ https://doi.org/10.1016/j.neuroimage.2016.12.052

Grüsser, O.J., Pause, M., Schreiter, U., 1990. Vestibular neurones in the parietoinsular cortex of monkeys (Macaca fascicularis): visual and neck recepto responses. The Journal of Physiology 430, 559-583. https://doi.org/10.1113/jphysiol.1990.sp018307

Gu, Y., 2018. Vestibular signals in primate cortex for self-motion perception. $\begin{array}{ccccc}\text { Gu, Y., 2018. Vestibular signals in primate cortex for self-motion perception. } \\ \text { Current } & \text { Opinion in } & \text { Neurobiology } & 52, & 10-17\end{array}$ https://doi.org/10.1016/j.conb.2018.04.004

Gu, Y., Angelaki, D.E., DeAngelis, G.C., 2008. Neural correlates of multisensory cue integration in macaque MSTd. Nat Neurosci 11, 1201-1210. https://doi.org/10.1038/nn.2191

Gu, Y., Cheng, Z., Yang, L., DeAngelis, G.C., Angelaki, D.E., 2016. Multisensory Convergence of Visual and Vestibular Heading Cues in the Pursuit Area of the Frontal Eye Field. Cereb. Cortex 26, 3785-3801. https://doi.org/10.1093/cercor/bhv183

Gu, Y., Deangelis, G.C., Angelaki, D.E., 2012. Causal links between dorsal media superior temporal area neurons and multisensory heading perception. J Neurosci 32, 2299-2313. https://doi.org/10.1523/JNEUROSCI.5154-11.2012

Gu, Y., DeAngelis, G.C., Angelaki, D.E., 2007. A functional link between area MSTd and heading perception based on vestibular signals. Nat Neurosci 10 1038-1047. https://doi.org/10.1038/nn1935

Gu, Y., Watkins, P.V., Angelaki, D.E., DeAngelis, G.C., 2006. Visual and Nonvisual Contributions to Three-Dimensional Heading Selectivity in the Media Superior Temporal Area. J. Neurosci. 26, 73-85. https://doi.org/10.1523/JNEUROSCI.2356-05.2006

Guipponi, O., Cléry, J., Odouard, S., Wardak, C., Ben Hamed, S., 2015. Whole brain mapping of visual and tactile convergence in the macaque monkey. NeuroImage 117, 93-102. https://doi.org/10.1016/j.neuroimage.2015.05.022 Guipponi, O., Wardak, C., Ibarrola, D., Comte, J.-C., Sappey-Marinier, D., Pinède, S., Ben Hamed, S., 2013. Multimodal convergence within the intraparietal sulcus of the macaque monkey. J. Neurosci. 33, 4128-4139. https://doi.org/10.1523/JNEUROSCI.1421-12.2013

Guldin, W.O., Grüsser, O.-J., 1998. Is there a vestibular cortex? Trends in Neurosciences 21, 254-259. https://doi.org/10.1016/S0166-2236(97)01211-3

Hart, E., Huk, A.C., 2020. Recurrent circuit dynamics underlie persistent activity in the macaque frontoparietal network. eLife 9, e52460. https://doi.org/10.7554/eLife.52460

Hatsopoulos, N.G., Suminski, A.J., 2011. Sensing with the Motor Cortex. Neuron 72, 477-487. https://doi.org/10.1016/j.neuron.2011.10.020

Heed, T., Beurze, S.M., Toni, I., Roder, B., Medendorp, W.P., 2011. Functional Rather than Effector-Specific Organization of Human Posterior Parietal Cortex J. Neurosci. 31, 3066-3076. https://doi.org/10.1523/JNEUROSCI.4370-10.2011 Heed, T., Habets, B., Sebanz, N., Knoblich, G., 2010. Others' Actions Reduce Crossmodal Integration in Peripersonal Space. Curr. Biol. 20, 1345-1349. https://doi.org/10.1016/j.cub.2010.05.068

Herrington, T.M., Masse, N.Y., Hachmeh, K.J., Smith, J.E.T., Assad, J.A., Cook, E.P., 2009. The effect of microsaccades on the correlation between neural activity and behavior in middle temporal, ventral intraparietal, and lateral intraparietal areas. J. Neurosci. 29, 5793-5805. https://doi.org/10.1523/JNEUROSCI.441208.2009

Hihara, S., Notoya, T., Tanaka, M., Ichinose, S., Ojima, H., Obayashi, S., Fujii, N., Iriki, A., 2006. Extension of corticocortical afferents into the anterior bank of the intraparietal sulcus by tool-use training in adult monkeys. Neuropsychologia, Visuomotor Functions of the Posterior Parietal Cortex 44, 2636-2646. https://doi.org/10.1016/j.neuropsychologia.2005.11.020

Hikosaka, K., Iwai, E., Saito, H., Tanaka, K., 1988. Polysensory properties of neurons in the anterior bank of the caudal superior temporal sulcus of the macaque $\begin{array}{llll}\text { monkey. J } & \text { Neurophysiol } & 60 & 1615-1637\end{array}$ https://doi.org/10.1152/jn.1988.60.5.1615

Hilgetag, C.-C., Essen, D.C.V., Hilgetag, C.-C., Essen, D.C.V., O'Neill, M.A., Young, M.P., Felleman, D.J., O’Neill, M.A., Young, M.P., Felleman, D.J., 1996. Indeterminate Organization of the Visual SystemOn Hierarchies: Response to Hilgetag et al.Rejoinder:FURTHER COMMENTARY: Determinate or Indeterminate Organization. Science 271, 776-776. https://doi.org/10.1126/science.271.5250.776

Hill, J., Inder, T., Neil, J., Dierker, D., Harwell, J., Van Essen, D., 2010. Simila patterns of cortical expansion during human development and evolution. PNAS 107, 13135-13140. https://doi.org/10.1073/pnas.1001229107

Huang, R.-S., Chen, C.-F., Sereno, M.I., 2018. Spatiotemporal integration of looming visual and tactile stimuli near the face. Hum Brain Mapp 39, 2156-2176. https://doi.org/10.1002/hbm.23995

Huang, R.-S., Chen, C.-F., Sereno, M.I., 2017. Mapping the complex topological organization of the human parietal face area. Neuroimage 163, 459-470. https://doi.org/10.1016/j.neuroimage.2017.09.004
Huang, R.-S., Chen, C.-F., Sereno, M.I., 2015. Neural substrates underlying the assive observation and active control of translational egomotion. J. Neurosci. 35 , 4258-4267. https://doi.org/10.1523/JNEUROSCI.2647-14.2015

Huang, R.-S., Chen, C.-F., Tran, A.T., Holstein, K.L., Sereno, M.I., 2012. Mapping multisensory parietal face and body areas in humans. Proceedings of the National $\begin{array}{lllll}\text { Academy } & \text { of } & \text { Sciences } & 109, & 18114-18119 \text {. }\end{array}$ https://doi.org/10.1073/pnas.1207946109

Huang, R.-S., Sereno, M.I., 2018. Multisensory and sensorimotor maps, in: Handbook of Clinical Neurology. Elsevier, pp. 141-161. https://doi.org/10.1016/B978-0-444-63622-5.00007-3

Huang, R.-S., Sereno, M.I., 2007. Dodecapus: An MR-compatible system for somatosensory stimulation. NeuroImage 34, 1060-1073. https://doi.org/10.1016/j.neuroimage.2006.10.024

Iriki, A., Tanaka, M., Iwamura, Y., 1996. Coding of modified body schema during tool use by macaque postcentral neurones. Neuroreport 7, 2325-2330. https://doi.org/10.1097/00001756-199610020-00010

Ishida, H., Nakajima, K., Inase, M., Murata, A., 2010. Shared Mapping of Own and Others' Bodies in Visuotactile Bimodal Area of Monkey Parietal Cortex. Journal of Cognitive Neuroscience 22, 83-96. https://doi.org/10.1162/jocn.2009.21185

Jacob, S.N., Nieder, A., 2014. Complementary roles for primate frontal and parietal cortex in guarding working memory from distractor stimuli. Neuron 83, 226-237. https://doi.org/10.1016/j.neuron.2014.05.009

Joly, O., Pallier, C., Ramus, F., Pressnitzer, D., Vanduffel, W., Orban, G.A., 2012a. Processing of vocalizations in humans and monkeys: A comparative fMRI study. NeuroImage 62, 1376-1389. https://doi.org/10.1016/j.neuroimage.2012.05.070 Joly, O., Ramus, F., Pressnitzer, D., Vanduffel, W., Orban, G.A., 2012b. Interhemispheric Differences in Auditory Processing Revealed by fMRI in Awake Rhesus Monkeys Cereb Cortex 22, 838-853. https://doi.org/10.1093/cercor/bhr150

Kaas, J.H., Stepniewska, I., 2016. The evolution of posterior parietal cortex and parietal-frontal networks for specific actions in primates. J Comp Neurol 524, 595-608. https://doi.org/10.1002/cne.23838

Kaminiarz, A., Schlack, A., Hoffmann, K.-P., Lappe, M., Bremmer, F., 2014. Visual selectivity for heading in the macaque ventral intraparietal area. J Neurophysiol 112, 2470-2480. https://doi.org/10.1152/jn.00410.2014

Kaneko, T., Tomonaga, M., 2012. Relative contributions of goal representation and kinematic information to self-monitoring by chimpanzees and humans. Cognition 125, 168-178. https://doi.org/10.1016/j.cognition.2012.07.006

Kaneko, T., Tomonaga, M., 2011. The perception of self-agency in chimpanzees (Pan troglodytes). Proceedings of the Royal Society B: Biological Sciences 278, 3694-3702. https://doi.org/10.1098/rspb.2011.0611

Kastner, S., Chen, Q., Jeong, S.K., Mruczek, R.E.B., 2017. A brief comparative review of primate posterior parietal cortex: A novel hypothesis on the human $\begin{array}{llll}\text { toolmaker. } & \text { Neuropsychologia } & 105, & 123-134 .\end{array}$ https://doi.org/10.1016/j.neuropsychologia.2017.01.034

Katz, L.N., Yates, J.L., Pillow, J.W., Huk, A.C., 2016. Dissociated functional significance of decision-related activity in the primate dorsal stream. Nature 535, 285-288. https://doi.org/10.1038/nature18617

Kilner, J.M., Lemon, R.N., 2013. What We Know Currently about Mirror Neurons. Curr. Biol. 23, R1057-R1062. https://doi.org/10.1016/j.cub.2013.10.051

Klam, F., Graf, W., 2006. Discrimination between active and passive head movements by macaque ventral and medial intraparietal cortex neurons. The Journal of Physiology 574, $367-386$. https://doi.org/10.1113/jphysiol.2005.103697

Klam, F., Graf, W., 2003. Vestibular response kinematics in posterior parietal cortex neurons of macaque monkeys. Eur. J. Neurosci. 18, 995-1010. https://doi.org/10.1046/j.1460-9568.2003.02813.x

Konen, C.S., Kastner, S., 2008a. Representation of Eye Movements and Stimulus Motion in Topographically Organized Areas of Human Posterior Parietal Cortex. J. Neurosci. 28, 8361-8375. https://doi.org/10.1523/JNEUROSCI.1930-08.2008 Konen, C.S., Kastner, S., 2008b. Two hierarchically organized neural systems for object information in human visual cortex. Nature Neuroscience 11, 224-231. https://doi.org/10.1038/nn2036

Konen, C.S., Kleiser, R., Seitz, R.J., Bremmer, F., 2005. An fMRI study of optokinetic nystagmus and smooth-pursuit eye movements in humans. Exp Brain Res 165, 203-216. https://doi.org/10.1007/s00221-005-2289-7

Kopp, K.S., Ebel, S.J., Wittig, R.M., Haun, D.B.M., Crockford, C., 2021. Small Mirrors Do the Trick: A Simple, but Effective Method to Study Mirror SelfRecognition in Chimpanzees. AB\&C 8, 391-404. https://doi.org/10.26451/abc.08.03.05.2021

Koyama, M., Hasegawa, I., Osada, T., Adachi, Y., Nakahara, K., Miyashita, Y., 2004. Functional Magnetic Resonance Imaging of Macaque Monkeys Performing Visually Guided Saccade Tasks: Comparison of Cortical Eye Fields with Humans. Neuron 41, 795-807. https://doi.org/10.1016/s0896-6273(04)00047-9

Krubitzer, L., 2009. In Search of a Unifying Theory of Complex Brain Evolution. Annals of the New York Academy of Sciences 1156, 44-67. https://doi.org/10.1111/j.1749-6632.2009.04421.x

Krubitzer, L., Disbrow, E., 2008. The evolution of parietal areas involved in hand use in primates, in: Kaas, J.H., Gardner, E. (Eds.), The Senses: A Comprehensive Reference. Elsevier, London, pp. 183-214.

Krubitzer, L.A., Prescott, T.J., 2018. The Combinatorial Creature: Cortical Phenotypes within and across Lifetimes. Trends in Neurosciences 41, 744-762. https://doi.org/10.1016/j.tins.2018.08.002

Lacadie, C.M., Fulbright, R.K., Rajeevan, N., Constable, R.T., Papademetris, X., 2008. More accurate Talairach coordinates for neuroimaging using non-linear registration. NeuroImage $42, \quad 717-725$. https://doi.org/10.1016/j.neuroimage.2008.04.240

Làdavas, E., Zeloni, G., Farnè, A., 1998. Visual peripersonal space centred on the face in humans. Brain 121, 2317-2326. https://doi.org/10.1093/brain/121.12.2317 
Lagae, L., Maes, H., Raiguel, S., Xiao, D.K., Orban, G.A., 1994. Responses of macaque STS neurons to optic flow components: a comparison of areas MT and MST. J Neurophysiol 71, 1597-1626. https://doi.org/10.1152/jn.1994.71.5.1597 Lane, A.R., Ball, K., Smith, D.T., Schenk, T., Ellison, A., 2013. Near and far space: Understanding the neural mechanisms of spatial attention. Hum. Brain Mapp. 34 356-366. https://doi.org/10.1002/hbm.21433

Leinonen, L., Hyvärinen, J., Sovijärvi, A.R.A., 1980. Functional properties of neurons in the temporo-parietal association cortex of awake monkey. Exp Brain Res 39, 203-215. https://doi.org/10.1007/BF00237551

Leoné, F.T.M., Monaco, S., Henriques, D.Y.P., Toni, I., Medendorp, W.P., 2015. Flexible Reference Frames for Grasp Planning in Human Parietofrontal Cortex eNeuro 2, ENEURO.0008-15.2015. https://doi.org/10.1523/ENEURO.000815.2015

Levitt, J.B., Kiper, D.C., Movshon, J.A., 1994. Receptive fields and functional architecture of macaque V2. J Neurophysiol 71, 2517-2542. https://doi.org/10.1152/jn.1994.71.6.2517

Lewis, J.W., Van Essen, D.C., 2000a. Mapping of architectonic subdivisions in the macaque monkey, with emphasis on parieto-occipital cortex. J. Comp. Neurol. $428,79-111$. https://doi.org/10.1002/1096-9861(20001204)428:1<79::aidcne7>3.0.co; $2-\mathrm{q}$

Lewis, J.W., Van Essen, D.C., 2000b. Corticocortical connections of visual, sensorimotor, and multimodal processing areas in the parietal lobe of the macaque monkey. J. Comp. Neurol. 428, 112-137. https://doi.org/10.1002/1096monkey. J. Comp. Neurol. 428, 112 -137.
9861(20001204)428:1<112::aid-cne8 $>3.0$. co; $2-9$

Lloyd, D.M., Shore, D.I., Spence, C., Calvert, G.A., 2003. Multisensory representation of limb position in human premotor cortex. Nat Neurosci 6,17 18. https://doi.org/10.1038/nn991

Maister, L., Cardini, F., Zamariola, G., Serino, A., Tsakiris, M., 2015. Your place or mine: shared sensory experiences elicit a remapping of peripersonal space. Neuropsychologia 70 ,

$455-461$ https://doi.org/10.1016/j.neuropsychologia.2014.10.027

Makin, T.R., Holmes, N.P., Ehrsson, H.H., 2008. On the other hand: Dummy hands and peripersonal space. Behavioural Brain Research 191, 1-10 https://doi.org/10.1016/j.bbr.2008.02.041

Makin, T.R., Holmes, N.P., Zohary, E., 2007. Is That Near My Hand? Multisensory Representation of Peripersonal Space in Human Intraparietal Sulcus. J. Neurosci. 27, 731-740. https://doi.org/10.1523/JNEUROSCI.3653-06.2007

Maravita, A., Iriki, A., 2004. Tools for the body (schema). Trends in Cognitive Sciences 8, 79-86. https://doi.org/10.1016/j.tics.2003.12.008

Maravita, A., Romano, D., 2018. The parietal lobe and tool use, in: Handbook of Clinical Neurology. Elsevier, pp. 481-498. https://doi.org/10.1016/B978-0-44463622-5.00025-5

Markov, N.T., Ercsey-Ravasz, M.M., Ribeiro Gomes, A.R., Lamy, C., Magrou, L., Vezoli, J., Misery, P., Falchier, A., Quilodran, R., Gariel, M.A., Sallet, J., Gamanut, R., Huissoud, C., Clavagnier, S., Giroud, P., Sappey-Marinier, D. Barone, P., Dehay, C., Toroczkai, Z., Knoblauch, K., Van Essen, D.C., Kennedy, H., 2014. A Weighted and Directed Interareal Connectivity Matrix for Macaque Cerebral Cortex. Cereb Cortex 24, 17-36. https://doi.org/10.1093/cercor/bhs270 Mars, R.B., Jbabdi, S., Sallet, J., O'Reilly, J.X., Croxson, P.L., Olivier, E., Noonan, M.P., Bergmann, C., Mitchell, A.S., Baxter, M.G., Behrens, T.E.J., JohansenBerg, H., Tomassini, V., Miller, K.L., Rushworth, M.F.S, 2011. DiffusionWeighted Imaging Tractography-Based Parcellation of the Human Parietal Cortex and Comparison with Human and Macaque Resting-State Functional

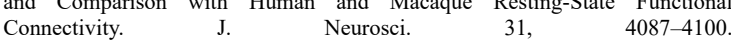
https://doi.org/10.1523/JNEUROSCI.5102-10.2011

Mars, R.B., Passingham, R.E., Jbabdi, S., 2018. Connectivity Fingerprints: From Areal Descriptions to Abstract Spaces. Trends in Cognitive Sciences 22, 10261037. https://doi.org/10.1016/j.tics. 2018.08.009

Mars, R.B., Sallet, J., Neubert, F.-X., Rushworth, M.F.S., 2013. Connectivity profiles reveal the relationship between brain areas for social cognition in human and monkey temporoparietal cortex. Proc Natl Acad Sci U S A 110, 10806-10811. https://doi.org/10.1073/pnas.1302956110

Martel, M., Ossandón, J.P., Habets, B., Heed, T., 2019. Protracted development of visuo-proprioceptive integration for uni-and bimanual motor coordination. bioRxiv 601435. https://doi.org/10.1101/601435

Maunsell, J.H.R., Van Essen, D.C., 1983. The connections of the middle temporal visual area (MT) and their relationship to a cortical hierarchy in the macaque monkey. J. Neurosci. 3, 2563-2586. https://doi.org/10.1523/JNEUROSCI.03-1202563.1983

Medendorp, W.P., Goltz, H.C., Vilis, T., Crawford, J.D., 2003. Gaze-Centered Updating of Visual Space in Human Parietal Cortex. J. Neurosci. 23, 6209-6214. https://doi.org/10.1523/JNEUROSCI.23-15-06209.2003

Medendorp, W.P. Heed, T., 2019. State estimation in posterior parietal cortex: Distinct poles of environmental and bodily states. Progress in Neurobiology 183 , 101691. https://doi.org/10.1016/j.pneurobio.2019.101691

Menz, V.K., Schaffelhofer, S., Scherberger, H., 2015. Representation of continuous hand and arm movements in macaque areas M1, F5, and AIP: a comparative decoding study. J. Neural Eng. 12, 056016. https://doi.org/10.1088/17412560/12/5/056016

Michaels, J.A., Scherberger, H., 2018. Population coding of grasp and lateralityrelated information in the macaque fronto-parietal network. Sci Rep 8, 1710 https://doi.org/10.1038/s41598-018-20051-7

Milham, M., Petkov, C.I., Margulies, D.S., Schroeder, C.E., Basso, M.A., Belin, P., Fair, D.A., Fox, A., Kastner, S., Mars, R.B., Messinger, A., Poirier, C., Vanduffel, W., Van Essen, D.C., Alvand, A., Becker, Y., Ben Hamed, S., Benn, A., Bodin, C., Boretius, S., Cagna, B., Coulon, O., El-Gohary, S.H., Evrard, H., Forkel, S.J., Friedrich, P., Froudist-Walsh, S., Garza-Villarreal, E.A., Gao, Y., Gozzi, A., Grigis, A., Hartig, R., Hayashi, T., Heuer, K., Howells, H., Ardesch, D.J., Jarraya, B., Jarrett, W., Jedema, H.P., Kagan, I., Kelly, C., Kennedy, H., Klink, P.C., Kwok, S.C., Leech, R., Liu, X., Madan, C., Madushanka, W., Majka, P., Mallon, A.-M., Marche, K., Meguerditchian, A., Menon, R.S., Merchant, H. Mitchell, A., Nenning, K.-H., Nikolaidis, A., Ortiz-Rios, M., Pagani, M., Pareek, V., Prescott, M., Procyk, E., Rajimehr, R., Rautu, I.-S., Raz, A., Roe, A.W., Rossi-
Pool, R., Roumazeilles, L., Sakai, T., Sallet, J., García-Saldivar, P., Sato, C., Sawiak, S., Schiffer, M., Schwiedrzik, C.M., Seidlitz, J., Sein, J., Shen, Z., Shmuel, A., Silva, A.C., Simone, L., Sirmpilatze, N., Sliwa, J., Smallwood, J., Tasserie, J., Thiebaut de Schotten, M., Toro, R., Trapeau, R., Uhrig, L., Vezoli, J., Wang, Z., Wells, S., Williams, B., Xu, T., Xu, A.G., Yacoub, E., Zhan, M., Ai, L., Amiez, C., Balezeau, F., Baxter, M.G., Blezer, E.L.A., Brochier, T., Chen, A., Croxson, P.L., Damatac, C.G., Dehaene, S., Everling, S., Fleysher, L., Freiwald, W., Griffiths, T.D., Guedj, C., Hadj-Bouziane, F., Harel, N., Hiba, B., Jung, B., Koo, B., Laland, K.N., Leopold, D.A., Lindenfors, P., Meunier, M., Mok, K., Morrison, J.H., Nacef, J., Nagy, J., Pinsk, M., Reader, S.M., Roelfsema, P.R., Rudko, D.A., Rushworth, M.F.S., Russ, B.E., Schmid, M.C., Sullivan, E.L., Thiele, A., Todorov, O.S., Tsao, D., Ungerleider, L., Wilson, C.R.E., Ye, F.Q., Zarco, W., Zhou, Y., 2020. Accelerating the Evolution of Nonhuman Primate Neuroimaging. Neuron 105, 600-603. https://doi.org/10.1016/j.neuron.2019.12.023

Milham, M.P., Ai, L., Koo, B., Xu, T., Amiez, C., Balezeau, F., Baxter, M.G., Blezer, E.L.A., Brochier, T., Chen, A., Croxson, P.L., Damatac, C.G., Dehaene, S., Everling, S., Fair, D.A., Fleysher, L., Freiwald, W., Froudist-Walsh, S., Griffiths, T.D., Guedj, C., Hadj-Bouziane, F., Ben Hamed, S., Harel, N., Hiba, B., Jarraya, B., Jung, B., Kastner, S., Klink, P.C., Kwok, S.C., Laland, K.N., Leopold, D.A., Lindenfors, P., Mars, R.B., Menon, R.S., Messinger, A., Meunier, M., Mok, K., Morrison, J.H., Nacef, J., Nagy, J., Rios, M.O., Petkov, C.I., Pinsk, M., Poirier, C., Procyk, E., Rajimehr, R., Reader, S.M. Roelfsema, P.R., Rudko, D.A., Rushworth, M.F.S., Russ, B.E., Sallet, J., Schmid, M.C., Schwiedrzik, C.M., Seidlitz, J., Sein, J., Shmuel, A., Sullivan, E.L., Ungerleider, L., Thiele, A., Todorov, O.S., Tsao, D., Wang, Z., Wilson, C.R.E., Yacoub, E., Ye, F.Q., Zarco, W., Zhou, Y., Margulies, D.S., Schroeder, C.E., 2018. An Open Resource for Nonhuman Primate Imaging. Neuron 100, 61-74.e2. https://doi.org/10.1016/j.neuron.2018.08.039

Morris, A.P., Bremmer, F., Krekelberg, B., 2016. The Dorsal Visual System Morris, A.P., Bremmer, F., Krekelberg, B., 2016. The Dorsal Visual System
Predicts Future and Remembers Past Eye Position. Front Syst Neurosci 10, 9. https://doi.org/10.3389/fnsys.2016.00009

Morris, A.P., Bremmer, F., Krekelberg, B., 2013. Eye-position signals in the dorsal visual system are accurate and precise on short timescales. J Neurosci 33, 1239512406. https://doi.org/10.1523/JNEUROSCI.0576-13.2013

Morris, A.P., Kubischik, M., Hoffmann, K.-P., Krekelberg, B., Bremmer, F., 2012. Dynamics of eye-position signals in the dorsal visual system. Curr. Biol. 22, 173179. https://doi.org/10.1016/j.cub.2011.12.032

Murata, A., Ishida, H., 2008. Consciousness of body and mirror neuron system. Acta Med Kinki Univ 33, 9-21.

Murata, A., Ishida, H., 2007. Representation of Bodily Self in the Multimodal Parieto-Premotor Network, in: Funahashi, S. (Ed.), Representation and Brain. Springer Japan, Tokyo, pp. 151-176. https://doi.org/10.1007/978-4-431-7302176

Newsome, W.T., Wurtz, R.H., Komatsu, H., 1988. Relation of cortical areas MT and MST to pursuit eye movements. II. Differentiation of retinal from extraretinal inputs. J Neurophysiol 60, 604-620. https://doi.org/10.1152/jn.1988.60.2.604 Nieder, A., 2012. Supramodal numerosity selectivity of neurons in primate prefrontal and posterior parietal cortices. Proceedings of the National Academy of Sciences 109, 11860-11865. https://doi.org/10.1073/pnas.1204580109

Nieder, A., Diester, I., Tudusciuc, O., 2006. Temporal and Spatial Enumeration Processes in the Primate Parietal Cortex. Science 313, 1431-1435. https://doi.org/10.1126/science. 1130308

Nieder, A., Miller, E.K., 2004. A parieto-frontal network for visual numerical information in the monkey. Proceedings of the National Academy of Sciences 101, 7457-7462. https://doi.org/10.1073/pnas.0402239101

Niu, M., Impieri, D., Rapan, L., Funck, T., Palomero-Gallagher, N., Zilles, K., 2020. Receptor-driven, multimodal mapping of cortical areas in the macaque monkey intraparietal sulcus. eLife 9, e55979. https://doi.org/10.7554/eLife.55979 Obayashi, S., Suhara, T., Kawabe, K., Okauchi, T., Maeda, J., Akine, Y., Onoe, H., Iriki, A., 2001. Functional Brain Mapping of Monkey Tool Use. NeuroImage 14, 853-861. https://doi.org/10.1006/nimg.2001.0878

Obayashi, S., Tanaka, M., Iriki, A., 2000. Subjective image of invisible hand coded by monkey intraparietal neurons. Neuroreport 11, 3499-3505. https://doi.org/10.1097/00001756-200011090-00020

Orban, G.A., 2016. Functional definitions of parietal areas in human and nonhuman primates. Proc. R. Soc. B. 283, 20160118. https://doi.org/10.1098/rspb.2016.0118

Orban, G.A., Van Essen, D., Vanduffel, W., 2004. Comparative mapping of higher visual areas in monkeys and humans. Trends in Cognitive Sciences 8, 315-324. https://doi.org/10.1016/j.tics.2004.05.009

Ortiz-Rios, M., Kuśmierek, P., DeWitt, I., Archakov, D., Azevedo, F.A.C., Sams, M. Jääskeläinen, I.P. Keliris, G.A., Rauschecker, J.P., 2015. Functional MRI of the vocalization-processing network in the macaque brain. Front. Neurosci. 9, 113. https://doi.org/10.3389/fnins.2015.00113

Padberg, J., Cerkevich, C., Engle, J., Rajan, A.T., Recanzone, G., Kaas, J., Krubitzer, L., 2009. Thalamocortical Connections of Parietal Somatosensory Cortical Fields in Macaque Monkeys are Highly Divergent and Convergent. Cereb Cortex 19, 2038-2064. https://doi.org/10.1093/cercor/bhn229

Paschke, K., Bähr, M., Wüstenberg, T., Wilke, M., 2019. Trunk rotation and handedness modulate cortical activation in neglect-associated regions during temporal order judgments. Neuroimage Clin 23, 101898. https://doi.org/10.1016/j.nicl.2019.101898

Paxinos, G., Huang, X.-F., Toga, A., 1999. The Rhesus Monkey Brain in Stereotaxic Coordinates. Academic Press, San Diego, California.

Paxinos, G., Watson, C., Petrides, M., Rosa, M., Tokuno, H., 2011. The Marmoset Brain in Stereotaxic Coordinates. Academic Press, San Diego, California.

Peel, T.R., Johnston, K., Lomber, S.G., Corneil, B.D., 2014. Bilateral saccadic deficits following large and reversible inactivation of unilateral frontal eye field. J Neurophysiol 111, 415-433. https://doi.org/10.1152/jn.00398.2013

Pellencin, E., Paladino, M.P., Herbelin, B., Serino, A., 2018. Social perception of others shapes one's own multisensory peripersonal space. Cortex 104, 163-179. https://doi.org/10.1016/j.cortex.2017.08.033 
Piazza, M., Izard, V., Pinel, P., Le Bihan, D., Dehaene, S., 2004. Tuning Curves for Approximate Numerosity in the Human Intraparietal Sulcus. Neuron 44, 547555. https://doi.org/10.1016/j.neuron.2004.10.014

Pitzalis, S., Fattori, P., Galletti, C., 2015. The human cortical areas V6 and V6A. Visual Neuroscience 32. https://doi.org/10.1017/S0952523815000048

Pitzalis, S., Hadj-Bouziane, F., Dal Bò, G., Guedj, C., Strappini, F., Meunier, M., Farnè, A., Fattori, P., Galletti, C., 2021. Optic flow selectivity in the macaque parieto-occipital sulcus. Brain Struct Funct. https://doi.org/10.1007/s00429-021 02293-w

Pitzalis, S., Sdoia, S., Bultrini, A., Committeri, G., Di Russo, F., Fattori, P., Galletti, C., Galati, G., 2013a. Selectivity to Translational Egomotion in Human Brain Motion Areas. PLoS ONE 8, e60241. https://doi.org/10.1371/journal.pone.0060241

Pitzalis, S., Sereno, M.I., Committeri, G., Fattori, P., Galati, G., Tosoni, A., Galletti, C., 2013b. The human homologue of macaque area V6A. NeuroImage 82, 517530. https://doi.org/10.1016/j.neuroimage.2013.06.026

Pitzalis, S., Serra, C., Sulpizio, V., Committeri, G., de Pasquale, F., Fattori, P., Galletti, C., Sepe, R., Galati, G., 2020. Neural bases of self- and object-motion in a naturalistic vision. Hum Brain Mapp 41, 1084-1111. https://doi.org/10.1002/hbm.24862

Pitzalis, S., Serra, C., Sulpizio, V., Di Marco, S., Fattori, P., Galati, G., Galletti, C., 2019. A putative human homologue of the macaque area PEc. NeuroImage 202, 116092. https://doi.org/10.1016/j.neuroimage.2019.116092

Pouget, A., Dayan, P., Zemel, R., 2000. Information processing with population codes. Nature Reviews Neuroscience 1, 125-132. https://doi.org/10.1038/35039062

Prado, J., Clavagnier, S., Otzenberger, H., Scheiber, C., Kennedy, H., Perenin, M.T., 2005. Two Cortical Systems for Reaching in Central and Peripheral Vision. Neuron 48, 849-858. https://doi.org/10.1016/j.neuron.2005.10.010

Quallo, M.M., Price, C.J., Ueno, K., Asamizuya, T., Cheng, K., Lemon, R.N., Iriki, A., 2009. Gray and white matter changes associated with tool-use learning in macaque monkeys. PNAS 106, 18379-18384. https://doi.org/10.1073/pnas.0909751106

Quinlan, D.J., Culham, J.C., 2007. fMRI reveals a preference for near viewing in the human parieto-occipital cortex. NeuroImage 36, 167-187. https://doi.org/10.1016/j.neuroimage.2007.02.029

Ramirez-Cardenas, A., Moskaleva, M., Nieder, A., 2016. Neuronal Representation of Numerosity Zero in the Primate Parieto-Frontal Number Network. Curr. Biol. 26, 1285-1294. https://doi.org/10.1016/j.cub.2016.03.052

Ramirez-Cardenas, A., Nieder, A., 2019. Working memory representation of empty sets in the primate parietal and prefrontal cortices. Cortex 114, 102-114. https://doi.org/10.1016/j.cortex.2019.02.028

Rauschecker, J.P., 2013. Processing Streams in Auditory Cortex, in: Cohen, Y.E., Popper, A.N., Fay, R.R. (Eds.), Neural Correlates of Auditory Cognition, Springe Handbook of Auditory Research. Springer New York, New York, NY, pp. 7-43. https://doi.org/10.1007/978-1-4614-2350-8 2

Rauschecker, J.P., 2011. An expanded role for the dorsal auditory pathway in sensorimotor control and integration. Hearing Research 271, 16-25. https://doi.org/10.1016/j.heares.2010.09.001

Renzi, C., Bruns, P., Heise, K.-F., Zimerman, M., Feldheim, J.-F., Hummel, F.C. Röder, B., 2013. Spatial Remapping in the Audio-tactile Ventriloquism Effect: A TMS Investigation on the Role of the Ventral Intraparietal Area. Journal of Cognitive Neuroscience 25, 790-801. https://doi.org/10.1162/jocn_a_00362 Richter, M., Amunts, K., Mohlberg, H., Bludau, S., Eickhoff, S.B., Zilles, K. Caspers, S., 2019. Cytoarchitectonic segregation of human posterior intraparieta and adjacent parieto-occipital sulcus and its relation to visuomotor and cognitive functions. Cereb Cortex 29, 1305-1327. https://doi.org/10.1093/cercor/bhy245

Rideaux, R., Storrs, K.R., Maiello, G., Welchman, A.E., 2021. How multisensory neurons R. https://doi.org/10.1073/pnas.2106235118

Romanski, L.M., Tian, B., Fritz, J., Mishkin, M., Goldman-Rakic, P.S. Rauschecker, J.P., 1999. Dual streams of auditory afferents target multiple domains in the primate prefrontal cortex. Nat Neurosci 2, 1131-1136. https://doi.org/10.1038/16056

Rosano, C., Krisky, C.M., Welling, J.S., Eddy, W.F., Luna, B., Thulborn, K.R. Sweeney, J.A. 2002. Pursuit and Saccadic Eye Movement Subregions in Human Frontal Eye Field: A High-resolution fMRI Investigation. Cerebral Cortex 12 107-115. https://doi.org/10.1093/cercor/12.2.107

Russ, B.E., Petkov, C.I., Kwok, S.C., Zhu, Q., Belin, P., Vanduffel, W., Ben Hamed, S., 2021. Common functional localizers to enhance NHP \& cross-specie neuroscience imaging research. NeuroImage 237, 118203 https://doi.org/10.1016/j.neuroimage.2021.118203

Salimi, I., Brochier, T., Smith, A.M., 1999. Neuronal Activity in Somatosensory Cortex of Monkeys Using a Precision Grip. I. Receptive Fields and Discharge Patterns. J Neurophysiol 81, 845-857. https://doi.org/10.1152/jn.1999.81.2.845 Schaafsma, S.J., Duysens, J., 1996. Neurons in the ventral intraparietal area of awake macaque monkey closely resemble neurons in the dorsal part of the medial superior temporal area in their responses to optic flow patterns. J. Neurophysiol. 76, 4056-4068. https://doi.org/10.1152/jn.1996.76.6.4056

Schall, J.D., 2015. Visuomotor Functions in the Frontal Lobe. Annu. Rev. Vis. Sci. 1, 469-498. https://doi.org/10.1146/annurev-vision-082114-035317

Scheperjans, F., Eickhoff, S.B., Hömke, L., Mohlberg, H., Hermann, K., Amunts, K., Zilles, K., 2008. Probabilistic Maps, Morphometry, and Variability of Cytoarchitectonic Areas in the Human Superior Parietal Cortex. Cereb Cortex 18 , 2141-2157. https://doi.org/10.1093/cercor/bhm241

Schindler, A., Bartels, A., 2018. Integration of visual and non-visual self-motion cues during voluntary head movements in the human brain. NeuroImage 172 597-607. https://doi.org/10.1016/j.neuroimage.2018.02.006

Schlack, A., Hoffmann, K.-P., Bremmer, F., 2003. Selectivity of macaque ventral intraparietal area (area VIP) for smooth pursuit eye movements. The Journal of Physiology 551, 551-561. https://doi.org/10.1113/jphysiol.2003.042994
Schlack, A., Hoffmann, K.-P., Bremmer, F., 2002. Interaction of linear vestibular and visual stimulation in the macaque ventral intraparietal area (VIP). Eur. J. Neurosci. 16, 1877-1886. https://doi.org/10.1046/j.1460-9568.2002.02251.x Schlack, A., Sterbing-D’Angelo, S.J., Hartung, K., Hoffmann, K.-P., Bremmer, F., 2005. Multisensory Space Representations in the Macaque Ventral Intraparietal Area. J. Neurosci. 25, 4616-4625. https://doi.org/10.1523/JNEUROSCI.045505.2005

Schlag, J., Schlag-Rey, M., 2002. Through the eye, slowly; Delays and localization errors in the visual system. Nat Rev Neurosci 3, 191. https://doi.org/10.1038/nrn750

Schluppeck, D., Glimcher, P., Heeger, D.J., 2005. Topographic organization for delayed saccades in human posterior parietal cortex. J Neurophysiol 94, 13721384. https://doi.org/10.1152/jn.01290.2004

Schmitt, C., Schwenk, J.C.B., Schütz, A., Churan, J., Kaminiarz, A., Bremmer, F., 2021. Preattentive processing of visually guided self-motion in humans and $\begin{array}{llll}\text { monkeys. } & \text { Prog } & \text { Neurobiol } & 102117 .\end{array}$ https://doi.org/10.1016/j.pneurobio.2021.102117

Seelke, A.M.H., Padberg, J.J., Disbrow, E., Purnell, S.M., Recanzone, G., Krubitzer, L., 2012. Topographic Maps within Brodmann's Area 5 of Macaque Monkeys. Cereb Cortex 22, 1834-1850. https://doi.org/10.1093/cercor/bhr257 Sereno, M.I., Huang, R.-S., 2014. Multisensory maps in parietal cortex. Current Opinion in Neurobiology 24, 39-46. https://doi.org/10.1016/j.conb.2013.08.014 Sereno, M.I., Huang, R.-S., 2006. A human parietal face area contains aligned head-centered visual and tactile maps. Nat Neurosci 9, 1337-1343. https://doi.org/10.1038/nn1777

Sereno, M.I., Pitzalis, S., Martinez, A., 2001. Mapping of Contralateral Space in Retinotopic Coordinates by a Parietal Cortical Area in Humans. Science 294, 1350-1354. https://doi.org/10.1126/science. 1063695

Serino, A., 2019. Peripersonal space (PPS) as a multisensory interface between the individual and the environment, defining the space of the self. Neuroscience \& Biobehavioral Reviews $99, \quad 138-159$. https://doi.org/10.1016/j.neubiorev.2019.01.016

Serino, A., Alsmith, A., Costantini, M., Mandrigin, A., Tajadura-Jimenez, A., Lopez, C., 2013. Bodily ownership and self-location: components of bodily self$\begin{array}{llll}\text { consciousness. Conscious } \quad \text { Cogn } & 22, & 1239-1252 .\end{array}$ https://doi. org/10.1016/j.concog 2013.08 .013

Serra, C. Galletti, C., Di Marco, S., Fattori, P., Galati, G., Sulpizio, V., Pitzalis, S, 2019. Egomotion-related visual areas respond to active leg movements. Hum Brain Mapp 40, 3174-3191. https://doi.org/10.1002/hbm.24589

Silver, M.A., Ress, D., Heeger, D.J., 2005. Topographic Maps of Visual Spatial Attention in Human Parietal Cortex. J Neurophysiol 94, 1358-1371. https://doi.org/10.1152/jn.01316.2004

Snyder, L.H., 2000. Coordinate transformations for eye and arm movements in the brain. Current Opinion in Neurobiology 10, 747-754. https://doi.org/10.1016/S0959-4388(00)00152-5

Snyder, L.H., Grieve, K.L., Brotchie, P., Andersen, R.A., 1998. Separate body- and world-referenced representations of visual space in parietal cortex. Nature 394, 887-891. https://doi.org/10.1038/29777

Sood, M.R., Sereno, M.I., 2016. Areas activated during naturalistic reading comprehension overlap topological visual, auditory, and somatotomotor maps. Hum. Brain Mapp. 37, 2784-2810. https://doi.org/10.1002/hbm.23208

Stein, B.E., Stanford, T.R., 2008. Multisensory integration: current issues from the perspective of the single neuron. Nat Rev Neurosci 9, 255-266. https://doi.org/10.1038/nrn2331

Stepniewska, I., Fang, P.-C., Kaas, J.H., 2005. Microstimulation reveals specialized subregions for different complex movements in posterior parietal cortex of prosimian galagos. Proceedings of the National Academy of Sciences 102, 4878-4883. https://doi.org/10.1073/pnas.0501048102

Stoet, G., Snyder, L.H., 2004. Single Neurons in Posterior Parietal Cortex of Monkeys Encode Cognitive Set. Neuron 42, 1003-1012. https://doi.org/10.1016/j.neuron.2004.06.003

Sulpizio, V., Galati, G., Fattori, P., Galletti, C., Pitzalis, S., 2020. A common neural substrate for processing scenes and egomotion-compatible visual motion. Brain Struct Funct 225, 2091-2110. https://doi.org/10.1007/s00429-020-02112-8

Sun, L.D., Goldberg, M.E., 2016. Corollary Discharge and Oculomotor Proprioception: Cortical Mechanisms for Spatially Accurate Vision. Annu. Rev. Vis. Sci. 2, 61-84. https://doi.org/10.1146/annurev-vision-082114-035407 Sunkara, A., DeAngelis, G.C., Angelaki, D.E., 2016. Joint representation of translational and rotational components of optic flow in parietal cortex. PNAS 113, 5077-5082. https://doi.org/10.1073/pnas.1604818113

Sunkara, A., DeAngelis, G.C., Angelaki, D.E., 2015. Role of visual and non-visual cues in constructing a rotation-invariant representation of heading in parietal cortex. eLife 4, e04693. https://doi.org/10.7554/eLife.04693

Takahashi, T., Kansaku, K., Wada, M., Shibuya, S., Kitazawa, S., 2013. Neural Correlates of Tactile Temporal-Order Judgment in Humans: an fMRI Study. Cereb Cortex 23, 1952-1964. https://doi.org/10.1093/cercor/bhs179

Tanner, J.E., Byrne, R.W., 1993. Concealing facial evidence of mood: Perspectivetaking in a captive gorilla? Primates 34, 451-457. https://doi.org/10.1007/BF02382654

Teneggi, C., Canzoneri, E., di Pellegrino, G., Serino, A., 2013. Social modulation of peripersonal space boundaries. Curr. Biol. 23, 406-411. https://doi.org/10.1016/j.cub.2013.01.043

Teramoto, W., 2018. A behavioral approach to shared mapping of peripersonal space between oneself and others. Sci Rep 8, 5432. https://doi.org/10.1038/s41598-018-23815-3

Thura, D., Hadj-Bouziane, F., Meunier, M., Boussaoud, D., 2008. Hand position modulates saccadic activity in the frontal eye field. Behavioural Brain Research 186, 148-153. https://doi.org/10.1016/j.bbr.2007.07.035

Tsakiris, M., 2008. Looking for myself: current multisensory input alters self-face recognition. PLoS One 3, e4040. https://doi.org/10.1371/journal.pone.0004040 
Uesaki, M., Ashida, H., 2015. Optic-flow selective cortical sensory regions associated with self-reported states of vection. Front Psychol 6,775 https://doi.org/10.3389/fpsyg.2015.00775

Ugolini, G., Prevosto, V., Graf, W., 2019. Ascending vestibular pathways to parietal areas MIP and LIPv and efference copy inputs from the medial reticula formation: Functional frameworks for body representations updating and online movement guidance. Eur J Neurosci 50, 2988-3013. https://doi.org/10.1111/ejn.14426

Uhrig, L., Dehaene, S., Jarraya, B., 2014. A Hierarchy of Responses to Auditory Regularities in the Macaque Brain. J. Neurosci. 34, 1127-1132. Regularities in the Macaque Brain. J. N
https://doi.org/10.1523/JNEUROSCI.3165-13.2014

Vallentin, D., Bongard, S., Nieder, A., 2012. Numerical rule coding in the prefrontal, premotor, and posterior parietal cortices of macaques. J. Neurosci. 32, 6621-6630. https://doi.org/10.1523/JNEUROSCI.5071-11.2012

van der Veer, A.H., Alsmith, A.J.T., Longo, M.R., Wong, H.Y, Mohler, B J. 2018. Where am I in virtual reality? PLoS ONE 13, e0204358. https://doi.org/10.1371/journal.pone.0204358

Van Essen, D.C., Glasser, M.F., Dierker, D.L., Harwell, J., 2012a. Cortica Parcellations of the Macaque Monkey Analyzed on Surface-Based Atlases. Cereb Cortex 22, 2227-2240. https://doi.org/10.1093/cercor/bhr290

Van Essen, D.C., Glasser, M.F., Dierker, D.L., Harwell, J., Coalson, T., 2012b. Parcellations and Hemispheric Asymmetries of Human Cerebral Cortex Analyzed on Surface-Based Atlases. Cereb Cortex 22, 2241-2262. https://doi.org/10.1093/cercor/bhr291

Vijayakumar, S., Sallet, J., Verhagen, L., Folloni, D., Medendorp, W.P., Mars, R.B., 2018. Mapping multiple principles of parietal-frontal cortical organization using functional connectivity. Brain Struct Funct 224, 681-697. https://doi.org/10.1007/s00429-018-1791-1

Viswanathan, P., Nieder, A., 2017a. Comparison of visual receptive fields in the dorsolateral prefrontal cortex and ventral intraparietal area in macaques. Eur. J. Neurosci. 46, 2702-2712. https://doi.org/10.1111/ejn.13740

Viswanathan, P., Nieder, A., 2017b. Visual Receptive Field Heterogeneity and Functional Connectivity of Adjacent Neurons in Primate Frontoparietal Association Cortices. J. Neurosci. 37, 8919-8928. https://doi.org/10.1523/JNEUROSCI.0829-17.2017

Viswanathan, P., Nieder, A., 2015. Differential impact of behavioral relevance on quantity coding in primate frontal and parietal neurons. Curr. Biol. 25, 1259 1269. https://doi.org/10.1016/j.cub.2015.03.025

Viswanathan, P., Nieder, A., 2013. Neuronal correlates of a visual "sense of number" in primate parietal and prefrontal cortices. Proc. Natl. Acad. Sci. U.S.A 110, 11187-11192. https://doi.org/10.1073/pnas.1308141110

Wall, M.B., Smith, A.T., 2008. The Representation of Egomotion in the Human Brain. Curr. Biol. 18, 191-194. https://doi.org/10.1016/j.cub.2007.12.053

Wang, L., Uhrig, L., Jarraya, B., Dehaene, S., 2015. Representation of Numerical and Sequential Patterns in Macaque and Human Brains. Curr. Biol. 25, 1966 1974. https://doi.org/10.1016/j.cub.2015.06.035

Wang, X., Zhang, M., Cohen, I.S., Goldberg, M.E., 2007. The proprioceptive representation of eye position in monkey primary somatosensory cortex. Nature Neuroscience 10, 640-646. https://doi.org/10.1038/nn1878

Wardak, C., Ben Hamed, S., Olivier, E., Duhamel, J.-R., 2012. Differential effects of parietal and frontal inactivations on reaction times distributions in a visual search task. Front Integr Neurosci 6, 39. https://doi.org/10.3389/fnint.2012.00039 Wardak, C., Guipponi, O., Pinède, S., Ben Hamed, S., 2016. Tactile representation of the head and shoulders assessed by fMRI in the nonhuman primate. Neurophysiol 115, 80-91. https://doi.org/10.1152/jn.00633.2015

Wardak, C., Olivier, E., Duhamel, J.-R., 2011. The relationship between spatial attention and saccades in the frontoparietal network of the monkey. European Journal of Neuroscience 33, 1973-1981. https://doi.org/10.1111/j.14609568.2011.07710.x

Weiss, P.H., Marshall, J.C., Wunderlich, G., Tellmann, L., Halligan, P.W., Freund, H.-J., Zilles, K., Fink, G.R., 2000. Neural consequences of acting in near versus far space: a physiological basis for clinical dissociations. Brain 123, 2531-2541. https://doi.org/10.1093/brain/123.12.2531

Weiss, P.H., Marshall, J.C., Zilles, K., Fink, G.R., 2003. Are action and perception in near and far space additive or interactive factors? NeuroImage 18, 837-846. https://doi.org/10.1016/S1053-8119(03)00018-1

Zaidel, A., DeAngelis, G.C., Angelaki, D.E., 2017. Decoupled choice-driven and stimulus-related activity in parietal neurons may be misrepresented by choice probabilities. Nat Commun 8, 715. https://doi.org/10.1038/s41467-017-00766-3 Zajac, L., Burte, H., Taylor, H.A., Killiany, R., 2019. Self-reported navigation ability is associated with optic flow-sensitive regions' functional connectivity patterns during visual path integration. Brain Behav 9, e01236. patterns during visual path
https://doi.org/10.1002/brb3.1236

Zajac, L., Killiany, R., 2021. Activity Strength within Optic Flow-Sensitive Cortical Regions Is Associated with Visual Path Integration Accuracy in Aged Adults. Brain Sciences 11, 245. https://doi.org/10.3390/brainsci11020245

Zhang, M., Barash, S., 2000. Neuronal switching of sensorimotor transformations for antisaccades. Nature 408, 971-975. https://doi.org/10.1038/35050097

Zhang, T., Britten, K.H., 2011. Parietal area VIP causally influences heading perception during pursuit eye movements. J. Neurosci. 31, 2569-2575. https://doi.org/10.1523/JNEUROSCI.5520-10.2011

Zhang, T., Britten, K.H., 2010. The responses of VIP neurons are sufficiently sensitive to support heading judgments. J. Neurophysiol. 103, 1865-1873. https://doi.org/10.1152/jn.00401.2009

Zhang, T., Heuer, H.W., Britten, K.H., 2004. Parietal area VIP neuronal responses to heading stimuli are encoded in head-centered coordinates. Neuron 42, 993 1001. https://doi.org/10.1016/j.neuron.2004.06.008 